\title{
UTILIZAÇÃO DE ONDALETAS EM MODELOS DE ESPAÇO DE ESTADOS
}

\section{Eliana Zandonade}

\author{
TESE APRESENTADA
}

$\mathrm{AO}$

INSTITUTO DE MATEMÁTICA E ESTATÍSTICA

DA

UNIVERSIDADE DE SÃO PAULO

PARA A OBTENÇÃO DO GRAU

DE

DOUTOR EM ESTATÍSTICA

Área de Concentração: Estatística

Orientador: Prof. Dr. Pedro A. Morettin

São Paulo, 22 de novembro de 1999 


\title{
UTILIZAÇÃO DE ONDALETAS EM MODELOS DE ESPAÇO DE ESTADOS
}

\author{
Este exemplar corresponde à redação \\ final da tese devidamente corrigida, \\ e defendida por Eliana Zandonade e \\ aprovada pela Comissão Julgadora.
}

São Paulo, 22 de novembro de 1999

\section{Banca Examinadora}

Prof. Dr. Pedro A. Morettin (IME-USP) - Orientador

Profa. Dra. Mariane Streibel (IME-USP)

Prof. Dr. Reinaldo Castro Souza (PUC-RJ)

Profa. Dra. Silvia Lopes (UFRGS-RS)

Prof. Dr. Luiz K. Hotta (IMECC-UNICAMP) 
Por que és o avesso, do avesso, do avesso, do avesso.

Caetano Veloso, em Sampa. 


\section{AGRADECIMENTOS}

Ao meu orientador, Prof. Pedro A. Morettin, pelas reuniões às sextas-feiras, verdadeiras aulas de orientação e estímulo ao trabalho;

Às professoras Clélia M. Toloi e Mariane Streibel, pela troca de experiências e conversas enriquecedoras;

Aos colegas do Departamento de Estatística da UFES, em especial à Angélica e ao Mário pela amizade;

Aos colegas do doutorado; em especial, ao Cardoso, Chico e Antonieta, pelas aventuras vividas juntos;

Ao Roberto, pelos conversas existenciais;

À minha querida irmã Adriana, por quatro anos de convivência e por me ensinar tanto sobre a vida;

Ao meu querido irmão Ivan, por estar sempre presente na minha vida apesar da distância;

Ao Udno, por me mostrar o encanto de ter um irmão mais novo;

À Orliene e Ricardo, por me darem o prazer de ser tia coruja do Daniel e da Isabel;

Ao Dani, por me fazer tão feliz;

Ao papai e à mamãe, por tudo o que sou (ou quase tudo). 


\title{
RESUMO
}

Neste trabalho utilizamos Ondaletas em Modelos de Espaço de Estados via parâmetros associados à matriz de transição e matriz do sistema e em Séries Temporais com mudanças de regime. Apresentamos a formulação do modelo com ondaletas e utilizamos o Algoritmo EM e o Filtro de Kalman para a estimação dos parâmetros e do vetor de estado, respectivamente.

\begin{abstract}
In this work we use Wavelets in State Space Models to represent the parameters corresponding to the transition and system matrices, and in Time Series with changes in regime. We present the formulation of the models with wavelets and propose to estimate the models parameters and the state vector using the EM Algorithm and the Kalman Filter, respectively.
\end{abstract}




\section{Sumário}

1 Introdução 1

2 Modelos de Espaço de Estados 4

2.1 Introdução . . . . . . . . . . . . . . . . . . . . . 4

2.2 O Filtro de Kalman . . . . . . . . . . . . . . . . . . . . . . . . . . 8

2.3 Estimação dos Parâmetros . . . . . . . . . . . . . . . . . . . . 12

2.3.1 Algoritmos de Máxima Verossimilhança . . . . . . . . . . 12

2.3.2 Métodos Bayesianos . . . . . . . . . . . . . . . 18

2.4 Modelos em Espaço de Estados Não-Normais e Não-Lineares . . . 18

3 Ondaletas 20

3.1 Introdução . . . . . . . . . . . . . . . . . . . . . . 20

3.2 Ondaletas versus Análise de Fourier . . . . . . . . . . . . . . . 20

3.3 Definições . . . . . . . . . . . . . . . . . . . . . . . . . . 22

3.4 Propriedades . . . . . . . . . . . . . . . . . . . . . 24

3.5 Encolhimento de Ondaletas . . . . . . . . . . . . . . 25

3.6 Principais Ondaletas . . . . . . . . . . . . . . . . . . . . 27

4 Modelos com Ondaletas na Matriz de Transição 33

4.1 Introdução . . . . . . . . . . . . . . . . . . . . . . . . . . . . . . . . . . . . . 33

4.2 Formulação do Modelo . . . . . . . . . . . . . . . . . . . . 36

4.3 Estimação dos Parâmetros . . . . . . . . . . . . . . . . . . . . 37

4.4 Estudo de Simulação de Modelos AR(1) com Parâmetro Variando no Tempo . . . . . . . . . . . . . . . . . . . . 42

5 Modelos com Mudança de Regime $\quad \mathbf{5 8}$

5.1 Introdução . . . . . . . . . . . . . . . . . . . . . 58

5.2 Modelagem com Ondaletas . . . . . . . . . . . . . . 61 
5.3 Estimação dos Parâmetros . . . . . . . . . . . . . . . . . . . 62

5.4 Aplicação do Modelo com Ondaletas em Exemplo do Artigo Shumway e Stoffer (1991) . . . . . . . . . . . . . . . . 69

6 Modelos com Ondaletas na Matriz do Sistema 85

6.1 Introdução . . . . . . . . . . . . . . . . . . . . . . 85

6.2 Formulação do Modelo . . . . . . . . . . . . . . . . . . . . 86

6.3 Estimação dos Parâmetros . . . . . . . . . . . . . . . . . . . . 86

6.4 Problemas de Estimação . . . . . . . . . . . . . . . . . . . . . 88

6.5 Exemplos de Séries Simuladas com Ondaletas Haar em $F_{t}$. . . . 90

7 Propriedades dos Estimadores e Convergência do Algoritmo EM101

7.1 Introdução . . . . . . . . . . . . . . . . . . . . . . . . . . 101

7.2 Convergência do Algoritmo EM . . . . . . . . . . . . . . . . 102

7.3 Erros Padrões dos Estimadores . . . . . . . . . . . . . . . . 102

7.4 Método Bootstrap . . . . . . . . . . . . . . . . . . . . . 104

7.4 .1 Introdução . . . . . . . . . . . . . . . . . . . . . . . . . . . . . . . . . . . . . . . . . .

7.4 .2 Algoritmo Bootstrap . . . . . . . . . . . . . 105

8 Aplicação em Dados Reais e Conclusão 107

8.1 Introdução . . . . . . . . . . . . . . . . . . . . . . . 107

8.2 Aplicação: Spread Diário entre dois Títulos da Dívida Externa

Brasileira . . . . . . . . . . . . . . . . . . 107

8.3 Conclusão . . . . . . . . . . . . . . . . . . . . 113

$\begin{array}{lr}\text { Referências Bibliográficas } & 114\end{array}$ 


\section{Lista de Figuras}

3.1 Gráficos da Ondaleta Haar para $j=4$ e $k=1, \ldots, 8$. . . . . . 29

3.2 Gráficos da Ondaleta Haar para $j=5$ e $k=1, \ldots, 16$. . . . . . 29

3.3 Gráficos da Ondaleta Chapéu Mexicano para $j=4$ e $k=1, \ldots, 8$. 30

3.4 Gráficos da Ondaleta Chapéu Mexicano para $j=5$ e $k=1, \ldots, 16$. 30

3.5 Gráficos da Ondaleta Morlet para $j=4$ e $k=1, \ldots, 8 . \ldots 31$

3.6 Gráficos da Ondaleta Morlet para $j=5$ e $k=1, \ldots, 16$. . . . . 31

3.7 Gráficos da Ondaleta Shannon para $j=4$ e $k=1, \ldots, 8$. . . . . 32

3.8 Gráficos da Ondaleta Shannon para $j=5$ e $k=1, \ldots, 16$. . . . 32

4.1 Gráficos de $y_{t}, \theta_{t}$ e $G_{t}$ (Série 1) . . . . . . . . . . . . . 48

4.2 Gráficos das estimativas de $G_{t}$ para diversos modelos (Série 1). . . 48

4.3 Gráficos das estimativas de $G_{t}$ utilizando limiares para os modelos com $m=4$ (Série 1). . . . . . . . . . . . . . . . . . 49

4.4 Gráficos de $y_{t}, \theta_{t}$ e $G_{t}$ e suas respectivas estimativas para os melhores modelos (BIC) (Série 1). . . . . . . . . . . . . . . 49

4.5 Gráficos de $y_{t}, \theta_{t}$ e $G_{t}$ (Série 2) . . . . . . . . . . . . . 52

4.6 Gráficos das estimativas de $G_{t}$ para diversos modelos (Série 2). . . 52

4.7 Gráficos das estimativas de $G_{t}$ utilizando limiares para os modelos com $m=4$ (Série 2). . . . . . . . . . . . . . . . . 53

4.8 Gráficos de $y_{t}, \theta_{t}$ e $G_{t}$ e suas respectivas estimativas para os melhores modelos (BIC) (Série 2). . . . . . . . . . . . . . . . 53

4.9 Gráficos de $y_{t}, \theta_{t}$ e $G_{t}$ (Série 3). . . . . . . . . . . . 56

4.10 Gráficos das estimativas de $G_{t}$ para diversos modelos (Série 3). . 56

4.11 Gráficos das estimativas de $G_{t}$ utilizando limiares para os modelos com $m=4$ (Série 3). . . . . . . . . . . . . . . . 57

4.12 Gráficos de $y_{t}, \theta_{t}$ e $G_{t}$ e suas respectivas estimativas para os melhores modelos (BIC) (Série 3). . . . . . . . . . . . 
5.1 Gráficos das séries $y_{t_{1}}, y_{t_{2}}$ e $y_{t_{3}}$ e suas respectivas estimativas para os modelos (Exemplo 1) . . . . . . . . . . . . . . . .

5.2 Gráficos dos estados $\theta_{t_{1}}, \theta_{t_{2}}$ e $\theta_{t_{3}}$ e suas respectivas estimativas para os modelos (Exemplo 1). . . . . . . . . . . . . .

5.3 Gráficos das estimativas das probabilidades e probabilidades filtradas (Exemplo 1) . . . . . . . . . . . . . . . 75

5.4 Gráficos das séries $y_{t_{1}}, y_{t_{2}}$ e $y_{t_{3}}$ e suas respectivas estimativas para os modelos (Exemplo 2). . . . . . . . . . . . . . . . 77

5.5 Gráficos dos estados $\theta_{t_{1}}, \theta_{t_{2}}$ e $\theta_{t_{3}}$ e suas respectivas estimativas para os modelos (Exemplo 2). . . . . . . . . . . . . . . 78

5.6 Gráficos das estimativas das probabilidades e probabilidades filtradas (Exemplo 2). . . . . . . . . . . . . . . . . . .

5.7 Gráficos das séries $y_{t_{1}}, y_{t_{2}}$ e $y_{t_{3}}$ e suas respectivas estimativas para os modelos (Exemplo 3). . . . . . . . . . . . . . . .

5.8 Gráficos dos estados $\theta_{t_{1}}, \theta_{t_{2}}$ e $\theta_{t_{3}}$ e suas respectivas estimativas para os modelos (Exemplo 3). . . . . . . . . . . . .

5.9 Gráficos das estimativas das probabilidades e probabilidades filtradas (Exemplo 3). . . . . . . . . . . . . . . . . 83

6.1 Gráficos de $y_{t}, \theta_{t}$ e $F_{t}$ e suas respectivas estimativas para os modelos Haar $\mathrm{m}=2$ (Exemplo 1) . . . . . . . . . . . . . . . . 94

6.2 Gráficos de $y_{t}, \theta_{t}$ e $F_{t}$ e suas respectivas estimativas para os modelos Haar, $\mathrm{m}=3$ (Exemplo 1). . . . . . . . . . . . . .

6.3 Gráficos de $y_{t}, \theta_{t}$ e $F_{t}$ e suas respectivas estimativas para os modelos Haar, $\mathrm{m}=4$ (Exemplo 1). . . . . . . . . . . . . . .

6.4 Gráficos de $y_{t}, \theta_{t}$ e $F_{t}$ e suas respectivas estimativas para os modelos Haar, $\mathrm{m}=5$ (Exemplo 1). . . . . . . . . . . . . . 95

6.5 Gráficos de $y_{t}, \theta_{t}$ e $F_{t}$ e suas respectivas estimativas para os modelos Haar, $\mathrm{m}=6$ (Exemplo 1) . . . . . . . . . . . . . . . 96

6.6 Gráficos de $y_{t}, \theta_{t}$ e $F_{t}$ e suas respectivas estimativas para os modelos Haar, $\mathrm{m}=2$ (Exemplo 2) . . . . . . . . . . . . . . . . . . 98

6.7 Gráficos de $y_{t}, \theta_{t}$ e $F_{t}$ e suas respectivas estimativas para os modelos Haar, $\mathrm{m}=3$ (Exemplo 2). . . . . . . . . . . . . . . . . 98

6.8 Gráficos de $y_{t}, \theta_{t}$ e $F_{t}$ e suas respectivas estimativas para os modelos Haar, $\mathrm{m}=4$ (Exemplo 2) . . . . . . . . . . . . . . . . . . . 99

6.9 Gráficos de $y_{t}, \theta_{t}$ e $F_{t}$ e suas respectivas estimativas para os modelos Haar, $m=5$ (Exemplo 2). . . . . . . . . . . . . . . 
6.10 Gráficos de $y_{t}, \theta_{t}$ e $F_{t}$ e suas respectivas estimativas para os modelos Haar, $\mathrm{m}=6$ (Exemplo 2) . . . . . . . . . . . . . . 100

8.1 Gráfico da Série Spread Diário. . . . . . . . . . . . . . . . . 108

8.2 Gráficos das estimativas dos parâmetros dos modelos do capítulo 5. 111

8.3 Histogramas das amostras bootstrap. . . . . . . . . . . . . . . . . 112 


\section{Lista de Tabelas}

4.1 Número de parâmetros para $m=2, \ldots, 6 \ldots \ldots$. . . . . . . . 45

4.2 Resultados obtidos para a Série 1 (Capítulo 4) . . . . . . . . . 47

4.3 Resultados obtidos para a Série 2 (Capítulo 4) . . . . . . . . . 51

4.4 Resultados obtidos para a Série 3 (Capítulo 4) . . . . . . . . 55

5.1 Possíveis valores de $F_{t}$ para o exemplo Shumway e Stoffer (1991) . 70

5.2 Valores de $F_{t}$ para os exemplos considerados . . . . . . . . . 71

5.3 Resultados obtidos para o exemplo 1 (Capítulo 5) . . . . . . . . 76

5.4 Resultados obtidos para o exemplo 2 (Capítulo 5) . . . . . . . . 80

5.5 Resultados obtidos para o exemplo 3 (Capítulo 5) . . . . . . . . 84

6.1 Parâmetros dos modelos simulados . . . . . . . . . . . . . . . . . 91

6.2 Resultados obtidos para o Exemplo 1 (Capítulo 6) . . . . . . . 93

6.3 Resultados obtidos para o Exemplo 2 (Capítulo 6) . . . . . . . . 97

8.1 Resultados obtidos para a Série Spread (Capítulo 5) . . . . . . . 110 


\section{Capítulo 1}

\section{Introdução}

A Análise de Séries Temporais é uma área de pesquisa intensa dentro da Estatística. Tanto no domínio do tempo, quanto no domínio da freqüência, utilizando a abordagem clássica ou a bayesiana, as metodologias e as técnicas se aperfeiçoam e buscam em outras áreas da ciência artefatos para o seu desenvolvimento.

Foi assim com o Filtro de Kalman (Anderson e Moore, 1979), desenvolvido em aplicações da Engenharia Elétrica e hoje uma técnica incorporada nos Modelos de Espaço de Estados e com o bootstrap, uma técnica computacional já utilizada em aplicações de Séries Temporais. Ver Efron e Tibshirani (1993) e Franco (1998). Continuando esta tendência, encontramos as ondaletas, uma ferramenta contemporânea com usos relevantes em Estatística e mais recentemente em Séries Temporais.

As ondaletas se apresentam como alternativas a outros sistemas de funções usados como bases para representação de funções pertencentes a certos espaços. A contribuição importante das ondaletas é que elas são localizadas no tempo (ou espaço), permitindo uma boa representação para sinais não-estacionários (Morettin, 1997a).

O objetivo deste trabalho é utilizar ondaletas nos modelos de Espaço de Estados por meio de parâmetros associados à matriz de transição e matriz do sistema.

Os Modelos em Espaço de Estados baseiam-se em métodos recursivos desenvolvidos inicialmente por engenheiros para resolver problemas de controle. A equação do sistema (vetor de estado) define-se como a informação mínima nescessária para descrever o estado futuro do sistema a partir do estado presente. 
A representação do sistema em termos de espaço de estados é conhecida como markoviana (Murteira, Müller e Turkman, 1993).

Atualmente, os Modelos em Espaço de Estados envolvem várias terminologias e tipos de notação, tanto na Engenharia quanto na Estatística. Os estatísticos falam de modelos lineares dinâmicos na abordagem bayesiana (West e Harrison, 1997), e modelos estruturais na abordagem clássica (Harvey, 1990).

As vantagens de se utilizar esta modelagem em espaço de estados são muitas. Além de sua generalidade, isto é, conseguir representar uma gama enorme de estruturas de séries temporais, incluindo aquelas com observações perdidas e intervalos de tempo desiguais, também permite a utilização do Filtro de Kalman para calcular a função de verossimilhança de maneira simples.

Os próximos dois capítulos apresentam uma revisão de conceitos em Modelos de Espaço de Estados e em Ondaletas e trazem muitas referências que poderão ser consultadas para um aprofundamento nos assuntos.

No capítulo 2 apresentamos os conceitos básicos dos Modelos de Espaço de Estados, incluindo o Filtro de Kalman e métodos de estimação de parâmetros. Discutimos ainda idéias para modelos gaussianos e não-gaussianos.

No capítulo 3 resumimos as principais ferramentas de ondaletas utilizadas neste trabalho, como definições, propriedades e as técnicas de encolhimento de ondaletas.

Os três capítulos seguintes tratam das principais contribuições deste trabalho. Aplicamos ondaletas na matriz de transição do modelo de espaço de estados (Capítulo 4), utilizamos uma modelagem via ondaletas em modelos de mudanças de regime (Capítulo 5) e aplicamos ondaletas na matriz do sistema (Capítulo 6).

Em todos eles, apresentamos a formulação do modelo com ondaletas e utilizamos o Algoritmo EM e o Filtro de Kalman para a estimação dos parâmentros e do vetor de estado, respectivamente. A implementação de todos os programas foi feita na linguagem Fortran90 e os exemplos de cada capítulo foram simulados no pacote computacional S-Plus. Os problemas numéricos encontrados foram comentados e resolvidos com a utilização de subrotinas de programação não-linear.

O Algoritmo EM foi utilizado em todo trabalho para estimar parâmetros. Desta forma, resumimos no Capítulo 7 as principais propriedades dos estimadores obtidos e comentamos sobre a convergência do algoritmo. Incluímos neste 
capítulo uma seção sobre o método bootstrap, técnica computacionalmente intensiva que permite uma avaliação da variabilidade das estimativas utilizando somente os dados.

E finalmente o Capítulo 8 traz uma aplicação a dados reais e a implementação da técnica bootstrap aplicada aos parâmetros estimados, juntamente com as conclusões e propostas para trabalhos futuros. 


\section{Capítulo 2}

\section{Modelos de Espaço de Estados}

\subsection{Introdução}

A formulação de um modelo em espaço de estados de um processo estocástico é baseada na propriedade de sistemas markovianos que estabelece a independência do futuro do processo em relação ao passado, dado o presente. Nestes sistemas, o estado do processo contém toda a informação do passado utilizada para a predição do futuro.

Seja $y_{t}$ o processo representando a observação do sistema no instante $t$ e $\theta_{t}$ o vetor representando o estado do sistema no instante $t$. O modelo de espaço de estados é descrito por um sistema de duas equações que representam:

i) a forma pela qual as observações do processo são geradas em função do vetor de estados (equação das observações);

ii) a evolução dinâmica do vetor de estados não observado (equação do sistema ou equação de transição).

Segundo Harvey (1990), o objetivo da formulação em espaço de estados é definir $\theta_{t}$ de tal modo que ele contenha toda a informação relevante do sistema no tempo $t$ e tendo o menor número de elementos possível.

Analiticamente temos:

$$
\begin{aligned}
& y_{t}=F_{t} \theta_{t}+v_{t}, \\
& \theta_{t}=G_{t} \theta_{t-1}+w_{t},
\end{aligned}
$$


onde

$t=1, \ldots, n$

$y_{t}$ é o vetor de observações $(r \times 1)$;

$F_{t}$ é a matriz do sistema $(r \times p)$;

$\theta_{t}$ é o vetor de estado $(p \times 1)$;

$G_{t}$ é a matriz de transição $(p \times p)$;

$v_{t}$ é o vetor de erros associado às observações $(r \times 1)$, suposto um ruído branco gaussiano com matriz de covariâncias $V_{t}$;

$w_{t}$ é o vetor de erros associado ao vetor de estados $(p \times 1)$, suposto um ruído branco gaussiano com matriz de covariâncias $W_{t}$.

Além da suposição de ruídos brancos gaussianos para $v_{t}$ e $w_{t}$, assumimos que eles são não correlacionados entre si para todas as defasagens, isto é, $E\left(v_{t} \cdot w_{t-k}\right)=$ $0, k=0,1, \ldots$ e também que para o tempo $t=0, \theta_{0}$ tem distribuição normal com média $\mu$ e matriz de covariâncias $\Sigma$.

Deste modo, o processo $y_{t}$ fica totalmente definido uma vez conhecidas as quantidades $\left(F_{t}, G_{t}, V_{t}, W_{t}\right)$ que denominamos vetor de caracterização do modelo na notação de Harrison e Stevens (1976).

O vetor de caracterização do modelo, $\varphi=\left(F_{t}, G_{t}, V_{t}, W_{t}\right)$, pode depender de um conjunto de parâmetros desconhecidos e uma das tarefas da Estatística é a estimação desses parâmetros.

O modelo de espaço de estados descrito pelas equações em (2.1) é uma forma geral de representar diversos modelos. Ilustramos este fato com alguns exemplos.

Exemplo 1:

Podemos interpretar o sistema (2.1) como um tipo de modelo de efeitos aleatórios para séries temporais, onde o vetor de efeitos $\theta_{t}$ tem uma estrutura de correlação ao longo do tempo imposta por um modelo autoregressivo multivariado. Neste contexto, o modelo (2.1) é uma generalização do modelo autoregressivo $A R$ que considera tanto os ruídos observacionais quanto o ruído induzido pelo modelo.

Exemplo 2:

Seja o modelo $A R M A(p, q)$ aplicado a $y_{t}$, isto é, 


$$
y_{t}=\phi_{1} y_{t-1}+\ldots+\phi_{p} y_{t-p}+a_{t}-\theta_{1} a_{t-1}-\ldots-\theta_{q} a_{t-q},
$$

com $\operatorname{Var}\left(a_{t}\right)=\sigma_{a}^{2}$. Segundo Harvey e Phillips (1979), a formulação em espaço de estados tem como vetor de caracterização do modelo $\varphi=\left(F_{t}, G_{t}, V, W\right)$ tal que:

$$
\begin{aligned}
& F_{t}=\left[\begin{array}{lllll}
1 & 0 & \cdots & 0 & 0
\end{array}\right]^{\prime}, \quad F_{t} \in \mathbb{R}^{k}, \\
& G_{t}=\left(\begin{array}{ccccc}
\phi_{1} & 1 & 0 & \cdots & 0 \\
\phi_{2} & 0 & 1 & \cdots & 0 \\
\cdots & \cdots & \cdots & \cdots & \cdots \\
\phi_{k-1} & 0 & 0 & \cdots & 1 \\
\phi_{k} & 0 & 0 & \cdots & 0
\end{array}\right), \\
& V=0 \text {, } \\
& W=\sigma_{a}^{2} \theta \theta^{\prime} \mathrm{e} \\
& \theta=\left[\begin{array}{llll}
1 & -\theta_{1} & \cdots & -\theta_{k-1}
\end{array}\right]^{\prime},
\end{aligned}
$$

$\operatorname{com} k=\max \{p, q+1\}$.

Exemplo 3:

O Modelo Estrutural introduzido em Harvey (1984) e considerado a versão clássica dos Modelos Lineares Dinâmicos de Harrison e Stevens (Harrison e Stevens, 1976) é baseado na hipótese da existência de componentes básicos de tendência, sazonalidade, componente cíclica e ruído aleatório. A análise de uma série temporal através desses componentes básicos apresenta uma interpretação clara dos mesmos.

Seja o modelo univariado geral dado abaixo, com a presença de alguns dos componentes numa dada série temporal $y_{t}$ :

$$
y_{t}=\mu_{t}+\gamma_{t}+\epsilon_{t}
$$

com

$\mu_{t}$ : componente de tendência; 
$\gamma_{t}$ : componente sazonal dada por fatores

$$
\sum_{j=0}^{s-1} \gamma_{t-j}=\omega_{t}
$$

$\gamma_{t-j}$ : fator sazonal correspondente ao instante $(t-j), j=0,1, \cdots, s-1$;

$s$ : comprimento da sazonalidade;

$\omega_{t}$ : perturbação aleatória associada à sazonalidade no instante $t$ e supostamente com as seguintes propriedades:

$\omega_{i}$ tem distribuição aproximadamente $N\left(0, V_{\omega}\right)$ e $E\left(\omega_{i} . \omega_{j}\right)=0, i \neq j, \quad i, j=$ $1,2, \cdots, n \mathrm{e}$

$\epsilon_{t}:$ perturbação das observações.

Podemos representar o modelo acima na forma de espaço de estados com

$$
\begin{gathered}
F_{t}=\left[\begin{array}{ccccccc}
1 & 0 & \vdots & 1 & 0 & \cdots & 0
\end{array}\right]^{\prime}, F_{t} \in \mathbb{R}^{s+1}, \\
G_{t}=\left(\begin{array}{ccccccc}
1 & 1 & \vdots & 0 & 0 & \cdots & 0 \\
0 & 1 & \vdots & 0 & 0 & \cdots & 0 \\
\cdots & \cdots & \cdots & \cdots & \cdots & \cdots & \cdots \\
0 & 0 & \vdots & -1 & -1 & \cdots & -1 \\
0 & 0 & \vdots & 1 & 0 & \cdots & 0 \\
0 & 0 & \vdots & 0 & 1 & \cdots & 0 \\
\cdots & \cdots & \vdots & \cdots & \cdots & \cdots & \cdots \\
0 & 0 & \vdots & 0 & 0 & \cdots & 1
\end{array}\right), G_{t} \in \mathbb{R}^{(s+1) \times(s+1)}, \\
\\
W_{t}=\left(\begin{array}{ccccccc}
V_{\mu} & 0 & \vdots & 0 & 0 & \cdots & 0 \\
0 & V_{\beta} & \vdots & 0 & 0 & \cdots & 0 \\
\cdots & \cdots & \cdots & \cdots & \cdots & \cdots & \cdots \\
0 & 0 & \vdots & V_{w} & 0 & \cdots & 0 \\
0 & 0 & \vdots & 0 & 0 & \cdots & 0 \\
\cdots & \cdots & \cdots & \cdots & \cdots & \cdots & \cdots \\
0 & 0 & \vdots & 0 & 0 & \cdots & 0
\end{array}\right) \mathrm{e}
\end{gathered}
$$




$$
\theta_{t}=\left[\begin{array}{lllllll}
\mu_{t} & \beta_{t} & \vdots & \gamma_{t} & \gamma_{t-1} & \cdots & \gamma_{t-s-2}
\end{array}\right]^{\prime} ; \theta_{t} \in \mathbb{R}^{(s+1)} .
$$

Para operacionalizar o modelo estrutural, em vez de tentarmos ajustar funções que descrevam os componentes, podemos identificar a presença deles, utilizando a forma em espaço de estados e o filtro de Kalman, que é um procedimento pelo qual obtemos estimadores atualizados das componentes não observáveis em todo instante de tempo, a partir da informação trazida por $y_{t}$.

Tanto na abordagem "clássica" de Harvey, quanto na "bayesiana" de Harrison e Stevens, utiliza-se o procedimento de atualização sequencial via Filtro de Kalman. As diferenças entre as abordagens estão relacionadas com a natureza da estatística em que elas se baseiam.

Em Souza (1989) encontramos um resumo histórico desses modelos, tanto na abordagem clássica quanto na bayesiana.

A seção 2.2 apresenta o Filtro de Kalman com suas equações e propriedades. A seção 2.3 apresenta a estimação dos parâmetros contidos no vetor de caracterização do modelo e traz um resumo de alguns procedimentos de máxima verossimilhança e métodos bayesianos de estimação. E finalmente a seção 2.4 apresenta comentários sobre modelos em espaço de estados não-normais e não-lineares.

\subsection{O Filtro de Kalman}

O Filtro de Kalman é um procedimento recursivo para calcular o estimador ótimo do vetor de estado no tempo $t$ baseado nas informações até o tempo $t$. As informações consistem das observações, incluindo $y_{t}$.

O procedimento do Filtro de Kalman pode ser dividido em duas etapas:

i) Na primeira, deseja-se obter o melhor estimador da observação no instante $t$ usando toda a informação até o instante anterior. Corresponde a obter o melhor preditor através das equações de predição.

ii) Na segunda, o conhecimento da nova observação disponível é usado para atualizar o preditor obtido na etapa anterior. Isto é feito através das equações de atualização. 
O vetor de caracterização do modelo, $\varphi=\left(F_{t}, G_{t}, V_{t}, W_{t}\right)$ e $\mu$ e $\Sigma$ (média e matriz de covariâncias do vetor de estado no tempo $t=0$, respectivamente) são assumidos serem conhecidos em todos os períodos de tempo.

A média de $\theta_{t}$ (obtida pelo Filtro de Kalman) supondo a hipótese de normalidade, é um estimador ótimo de $\theta_{t}$ no sentido da minimização do erro quadrático médio. Se não tivermos a hipótese de normalidade, o Filtro de Kalman fornece o estimador ótimo no sentido da minimização do erro quadrático médio dentro da classe de todos os estimadores lineares (Harvey, 1990).

Mesmo com a hipótese de normalidade, sabe-se que os modelos utilizando o Filtro de Kalman não são robustos. Em Meinhold e Singpurwalla (1989) apresenta-se um método de robustificação do Filtro de Kalman.

\section{Derivação do Filtro de Kalman}

Sejam as seguintes notações para as esperanças e covariâncias do vetor de espaços de estados, condicionais ao vetor das observações:

i) $x_{t}^{s}=E\left(\theta_{t} \mid y_{1}, \ldots, y_{s}\right)$;

ii) $P_{t}^{s}=\operatorname{var}\left(\theta_{t} \mid y_{1}, \ldots, y_{s}\right)$;

iii) $P_{t, t-1}^{s}=\operatorname{cov}\left(\theta_{t}, \theta_{t-1} \mid y_{1}, \ldots, y_{s}\right)$.

Então podemos definir:

- $x_{t}^{t}$ e $P_{t}^{t}$ : estimadores obtidos pelo Filtro de Kalman usual;

- $x_{t}^{n}$ e $P_{t}^{n}, t \leq n$ : estimadores suavizados de erro quadrático médio mínimo (EQMM) de $x_{t}$ baseado em todas as observações $y_{1}, \ldots, y_{n}$;

- $x_{t}^{n}$ e $P_{t}^{n}, t>n$ : estimadores para previsão da série $\theta_{t}$.

Apresentamos a seguir os resultados das equações do Filtro de Kalman, onde é permitido que $F_{t}, G_{t}, V_{t}$ e $W_{t}$ variem com o tempo. Detalhes podem ser encontrados em Anderson e Moore (1979).

Equações de Predição e Atualização: para $t=1, \ldots, n$.

- Instante $(t-1)$ :

Seja o instante genérico $(t-1)$ no qual conhecemos:

i) $x_{t-1}^{t-1}=E\left(\theta_{t-1} \mid y_{1}, \ldots, y_{t-1}\right) \mathrm{e}$ 
ii) $P_{t-1}^{t-1}=\operatorname{cov}\left(\theta_{t-1} \mid y_{1}, \ldots y_{t-1}\right)$.

- Instante $t$ :

Antes de recebermos a informação $y_{t}$, podemos calcular as características do estimador que minimiza o erro quadrático médio (EQM) de $\theta_{t}$ dado o conhecimento do sistema até $t-1$. Sejam

i) $x_{t}^{t-1}=E\left(\theta_{t} \mid y_{1}, \ldots, y_{t-1}\right) \mathrm{e}$

ii) $P_{t}^{t-1}=\operatorname{cov}\left(\theta_{t} \mid y_{1}, \ldots y_{t-1}\right)$.

Então,

$$
\begin{gathered}
x_{t}^{t-1}=G_{t} x_{t-1}^{t-1} \\
P_{t}^{t-1}=G_{t} P_{t-1}^{t-1} G_{t}^{\prime}+W_{t} .
\end{gathered}
$$

Observando $y_{t}$ no instante $t$, podemos atualizar os estimadores $x_{t}^{t-1}$ e $P_{t}^{t-1}$ do vetor de estado no instante $t$, isto é, calcular:

i) $x_{t}^{t}=E\left(\theta_{t} \mid y_{1}, \ldots, y_{t}\right) \mathrm{e}$

ii) $P_{t}^{t}=\operatorname{cov}\left(\theta_{t} \mid y_{1}, \ldots y_{t}\right)$ obtidos através das equações recursivas abaixo:

$$
\begin{gathered}
x_{t}^{t}=x_{t}^{t-1}+K_{t}\left(y_{t}-F_{t} x_{t}^{t-1}\right) \quad \mathrm{e} \\
P_{t}^{t}=P_{t}^{t-1}-K_{t} F_{t} P_{t}^{t-1},
\end{gathered}
$$

onde

$$
K_{t}=P_{t}^{t-1} F_{t}^{\prime}\left(F_{t} P_{t}^{t-1} F_{t}^{\prime}+V_{t}\right)^{-1} \text { é o ganho do filtro, } x_{0}^{0}=\mu \text { e } P_{0}^{0}=\Sigma .
$$

As equações (2.2) a (2.5) constituem o Filtro de Kalman, que permite a atualização seqüencial do vetor de estados no instante $t-1$ para o instante $t$.

No contexto bayesiano, podemos interpretar o procedimento acima como:

- Posteriori no instante $(t-1)$ : (conhecida)

$$
\left(\theta_{t-1} \mid t-1\right) \sim N\left(x_{t-1}^{t-1}, P_{t-1}^{t-1}\right)
$$


- Priori no instante $t$ : (calculada)

$\left(\theta_{t} \mid t-1\right) \sim N\left(x_{t}^{t-1}, P_{t}^{t-1}\right)$

- Posteriori no instante $t$ : (calculada pelo teorema de Bayes)

$\left(\theta_{t} \mid t\right) \sim N\left(x_{t}^{t}, P_{t}^{t}\right)$

Observação: A operacionalização seqüencial do Filtro de Kalman requer que os valores $\mu$ e $\Sigma$ sejam conhecidos a priori. Estes podem ser calculados a partir das $k$ primeiras observações ou por outros métodos descritos na próxima seção.

Equações de Suavização: para $t=n, n-1, \ldots, 1$ :

Se estivermos interessados nos estimadores do vetor de estado correspondente ao período amostrado, estes podem ser obtidos recursivamente aplicando o Filtro de Kalman em "sentido inverso".

Consideramos o último estimador do vetor de estado obtido pela passagem do Filtro de Kalman pelas observações disponíveis $\left(y_{1}, \ldots, y_{n}\right)$ como valores iniciais para uma recursão inversa do filtro. As equações abaixo apresentam os estimadores suavizados ótimos do vetor de estado. Este procedimento é conhecido como suavização ("smoothing") do vetor de estado.

$$
\begin{aligned}
& x_{t-1}^{n}=x_{t-1}^{t-1}+J_{t-1}\left(x_{t}^{n}-G_{t} x_{t-1}^{t-1}\right) \quad \mathrm{e} \\
& P_{t-1}^{n}=P_{t-1}^{t-1}+J_{t-1}\left(P_{t}^{n}-P_{t}^{t-1}\right) J_{t-1}^{\prime}
\end{aligned}
$$

onde

$$
\begin{aligned}
& J_{t-1}=P_{t-1}^{t-1} G_{t}^{\prime}\left(P_{t}^{t-1}\right)^{-1} \\
& P_{t-1, t-2}^{n}=P_{t-1}^{t-1} J_{t-2}^{\prime}+J_{t-1}\left(P_{t, t-1}^{n}-G_{t} P_{t-1}^{t-1}\right) J_{t-2}^{\prime} \\
& P_{n, n-1}^{n}=\left(I-K_{n} F_{n}\right) G_{t} P_{n-1}^{n-1} \mathrm{e} \\
& \mathrm{I}=\text { matriz identidade. }
\end{aligned}
$$




\subsection{Estimação dos Parâmetros}

Uma vez que os valores suavizados obtidos pelo Filtro de Kalman dependem dos valores iniciais considerados para o vetor de parâmetros $\varphi$, é importante considerar várias metodologias para a obtenção de estimadores.

Seja $\varphi=(F, G, V, W)$ o vetor de caracterização do modelo, onde cada elemento não varia com o tempo e $\mu$ e $\Sigma$ a média e a covariância do vetor de estado no tempo $t=0$, respectivamente. Segundo Harvey (1990), o modelo acima é dito invariante no tempo ou homogêneo no tempo. Modelos estacionários são um caso especial.

Em muitos casos, os métodos de estimação por máxima verossimilhança utilizam técnicas de escore, Newton-Raphson e o algoritmo E.M. Pode-se pensar também em métodos bayesianos de estimação. Apresentamos a seguir um resumo dos principais procedimentos para a estimação desses parâmetros.

\subsubsection{Algoritmos de Máxima Verossimilhança}

A teoria clássica da máxima verossimilhança é baseada na situação onde temos um conjunto de $T$ observações $\left(y_{1}, \ldots, y_{T}\right)$ independentes e identicamente distribuídas.

A função de densidade conjunta é definida por:

$$
L(y ; \varphi)=\prod_{t=1}^{T} P\left(y_{t}\right)
$$

onde $P\left(y_{t}\right)$ é a função densidade de probabilidade conjunta do t-ésimo grupo de observações.

Como as observações já foram obtidas, $L(y ; \varphi)$ é interpretada como uma função de verossimilhança e o estimador de máxima verossimilhança é encontrado maximizando esta função com relação a $\varphi$.

Em séries temporais, as observações não são independentes e usamos a definição de função densidade de probabilidade condicional para escrever a função densidade conjunta como 


$$
L(y ; \varphi)=\prod_{t=1}^{T} P\left(y_{t} \mid Y_{t-1}\right),
$$

onde $Y_{t-1}=\left\{y_{t-1}, \ldots, y_{1}\right\}$.

Para o modelo Normal, a função log verossimilhança, considerando os resultados obtidos pelo Filtro de Kalman, é:

$$
\log L=-\frac{N T}{2} \log 2 \pi-\frac{1}{2} \sum_{t=1}^{T} \log \left|C_{t}\right|-\frac{1}{2} \sum_{t=1}^{T} \nu_{t}^{\prime} F_{t}^{-1} \nu_{t}
$$

onde

$$
\begin{aligned}
& C_{t}=\operatorname{cov}\left(y_{t}\right)=F \cdot P_{t}^{t-1} \cdot F^{\prime}+V, \\
& \nu_{t}=y_{t}-\hat{y}_{t / t-1}(\text { erro de predição) e } \\
& \hat{y}_{t / t-1}=E\left(y_{t}\right)=F \cdot x_{t}^{t-1} .
\end{aligned}
$$

De um modo geral, podemos resumir o procedimento de obtenção de estimadores de máxima verossimilhança nos seguintes passos:

PASSO 0: Dar um valor inicial para o vetor $\varphi=(F, G, V, W), \mu$ e $\Sigma$;

PASSO 1: Aplicar o Filtro de Kalman;

PASSO 2: Calcular a função de verossimilhança para $y_{1}, \ldots, y_{n}$ e utilizar o algoritmo escore ou calcular a função de verossimilhança conjunta de $\theta_{0}, \ldots, \theta_{n}$ e $y_{1}, \ldots, y_{n}$ e utilizar o algoritmo EM para atualizar os parâmetros $\varphi=\{F, G, V, W\}$, considerando outros métodos para atualizar $\mu$ e $\Sigma$;

PASSO 3: Calcular a diferença entre os parâmetros e os parâmetros do passo anterior ou a diferença entre as funções de verossimilhança e voltar ao PASSO 1 até que o algoritmo convirja.

A seguir apresentaremos um resumo de métodos de estimação, o Algoritmo Escore, o Algoritmo EM e métodos alternativos de estimação de $\mu$ e $\Sigma$.

\section{i) Algoritmo Escore}

Segundo Harvey (1990), para o modelo (2.1) a atualização dos parâmetros é dada pela seguinte equação: 


$$
\varphi^{*}=\varphi-I(\varphi)^{-1} \cdot \frac{\partial \log L}{\partial \varphi}
$$

onde $I_{i j}(\varphi)$ é o elemento $(i, j)$ da matriz de informação de Fisher dada por

$$
I_{i j}(\varphi)=\frac{1}{2} \cdot \sum_{t=1}^{n}\left[\operatorname{tr}\left(C_{t}^{-1} \cdot \frac{\partial C_{t}}{\partial \varphi_{i}} \cdot C_{t}^{-1} \cdot \frac{\partial C_{t}}{\partial \varphi_{j}}\right)\right]+E\left[\sum_{t=1}^{n}\left(\frac{\partial \nu_{t}}{\partial \varphi_{i}}\right)^{\prime} \cdot C_{t}^{-1} \cdot \frac{\partial \nu_{t}}{\partial \varphi_{j}}\right]
$$

$C_{t}, \nu_{t}$ e $\hat{y}_{t / t-1}$ definidos acima;

$x_{t}^{t-1}$ e $P_{t}^{t-1}$ são obtidos pelo Filtro de Kalman;

$\frac{\partial \log L}{\partial \varphi_{i}}$ é o elemento $i$ do vetor escore, dado por:

$$
\frac{\partial \log L}{\partial \varphi_{i}}=-\frac{1}{2} \cdot \sum_{t=1}^{n}\left[\operatorname{tr}\left(C_{t}^{-1} \cdot \frac{\partial C_{t}}{\partial \varphi_{i}}\right)\left(I-C_{t}^{-1} \nu_{t} \nu_{t}^{-1}\right)\right]-\frac{\partial \nu_{t}^{\prime}}{\partial \varphi_{i}} \cdot C_{t}^{-1} \cdot \nu_{t}
$$

\section{ii) Algoritmo EM}

O Algoritmo de Esperança-Maximização ou Algoritmo EM é um algoritmo de otimização não linear apropriado para aplicações em modelos envolvendo componentes não observadas ou dados observados irregularmente ao longo do tempo. É um procedimento iterativo para calcular o estimador de máxima verossimilhança quando apenas um subconjunto do conjunto total dos dados é conhecido.

Dempster, Laird e Rubin (1977) demonstraram a aplicabilidade do algoritmo EM e são os responsáveis pela popularização deste método em Estatística. Detalhes da convergência e performance do algoritmo serão discutidos no Capítulo 7.

$\mathrm{Na}$ formulação usual do algoritmo EM, o vetor de dados completo é composto da série observada $(Y)$ e do processo não observado $(\theta)$. Em muitas aplicações, $\theta$ consiste de um processo "latente" ou processo não observado.

No modelo em espaço de estado, os dados $y_{1}, \ldots, y_{n}$ consistem no vetor observado e $\theta_{1}, \ldots, \theta_{n}$ no vetor de estado não observado. A aplicação do algoritmo EM neste modelo requer o estimador de erros quadráticos médios mínimos (EQMM) do vetor de estado, baseado no conjunto de todas as observações $n, t=1, \ldots, n$. 
Este estimador será denotado por $x_{t}^{n}, t=1, \ldots, n$ e a matriz de covariâncias, por $P_{t}^{n}, t=1, \ldots, n$. São obtidos pelo algoritmo de suavização, que requer uma passagem para frente e outra para trás nos dados (Filtro e Suavizador de Kalman, respectivamente).

Segundo Shumway e Stoffer (1982), o Algoritmo EM para o modelo na forma de espaço de estados é dado pelo seguinte procedimento:

PASSO E: Calcular a esperança da função log-verossimilhança conjunta de $\theta_{0}, \ldots, \theta_{n}$ e $y_{1}, \ldots, y_{n}$ dados $y_{1}, \ldots, y_{n}$, cuja expressão é

$$
E\left(\log L \mid y_{1}, \ldots, y_{n}\right) \doteq H(\mu, \Sigma, F, G, V, W)
$$

PASSO M: Maximizar $H(\mu, \Sigma, F, G, V, W)$ em relação aos parâmetros $\varphi=$ $(F, G, V, W)$. As soluções obtidas são:

$$
\begin{aligned}
F & =D \cdot C^{-1} \\
G & =B \cdot A^{-1} \\
V & =n^{-1}\left(C-B A^{-1} B^{\prime}\right) \\
W & =n^{-1} \sum_{t=1}^{n}\left[\left(y_{t}-F x_{t}^{n}\right)\left(y_{t}-F x_{t}^{n}\right)^{\prime}+F \cdot P_{t}^{n} \cdot F^{\prime}\right]
\end{aligned}
$$

onde

$$
\begin{aligned}
& A=\sum_{t=1}^{n}\left(P_{t-1}^{n}+x_{t-1}^{n} \cdot x_{t-1}^{n^{\prime}}\right), \\
& B=\sum_{t=1}^{n}\left(P_{t, t-1}^{n}+x_{t}^{n} \cdot x_{t-1}^{n^{\prime}}\right), \\
& C=\sum_{t=1}^{n}\left(P_{t}^{n}+x_{t}^{n} \cdot x_{t}^{n^{\prime}}\right), \\
& D=\sum_{t=1}^{n} x_{t}^{n} \cdot y_{t}^{\prime} \quad \mathrm{e} \\
& P_{t-1}^{n}, \quad P_{t, t-1}^{n}, P_{t}^{n}, x_{t}^{n} \text { são obtidos pelo suavizador de Kalman. }
\end{aligned}
$$

Do ponto de vista bayesiano, o algoritmo EM é um método iterativo para calcular a moda da distribuição de probabilidade a posteriori. Seja $\varphi^{i}$ a moda (no passo $i$ ) da distribuição a posteriori observada $p(\varphi \mid Y)$. Sejam $p(\varphi \mid Y, \theta$ ) a posteriori completa e $p\left(\theta \mid \varphi^{i}, Y\right)$ a distribuição condicional preditiva do processo 
latente $\theta$, condicional à moda no passo $i$ e aos dados. O passo E consiste em calcular

$$
Q\left(\varphi, \varphi^{i}\right)=\int_{\theta} \log [p(\varphi \mid Y, \theta)] p\left(\theta \mid \varphi^{i}, Y\right) d \theta
$$

isto é, a esperança de $\log [p(\varphi \mid Y, \theta)]$ em relação a $p\left(\theta \mid \varphi^{i}, Y\right)$.

E no passo $M$ a função $Q$ é maximizada em relação a $\varphi$ e obtém-se $\varphi^{i+1}$.

iii) Métodos de estimação de $\mu$ e $\Sigma$

Para estimar a média e a covariância do vetor de estado no tempo $t=0$, $\mu$ e $\Sigma$, respectivamente, existem métodos alternativos. Podemos considerar estes elementos como estocásticos, mas segundo Harvey (1990), algumas vezes é mais apropriado assumir valores fixos. Eles podem ser tratados como parâmetros adicionais que devem ser estimados como parte do procedimento de máxima verossimilhança. Um modo de proceder seria tratar os elementos de $\theta_{0}, \mu$ e $\Sigma$, como parte integrante do vetor de parâmetros $\varphi$ e maximizar a verossimilhança com respeito a $\left[\theta_{0}, \varphi\right]$. Este procedimento complica consideravelmente a otimização numérica (Harvey, 1990).

Uma solução prática é considerar que, condicional a $\varphi$, o estimador de $\theta_{0}$ é uma função linear das observações. Deste modo podemos concentrá-lo fora da função de verossimilhança, que seria maximizada apenas em relação aos elementos de $\varphi$. Harvey (1990) apresenta duas maneiras de fazer isso, considerando a seguinte particularização do modelo em (2.1): $E\left(\theta_{0}\right)=\mu$ (desconhecido) e $\operatorname{cov}\left(\theta_{0}\right)=\Sigma=0$. A primeira é utilizando um procedimento de mínimos quadrados generalizados (MQG) e a segunda é utilizando um algoritmo de Rosenberg. Ambos os procedimentos são apresentados em Harvey (1990) (seções (3.4.2) e (3.4.4), respectivamente).

Shumway, Olsen e Levy (1981) consideraram uma abordagem de estimação de verossimilhança e testaram hipóteses envolvendo o valor inicial da média $\mu$ e covariância $\Sigma$ do Filtro de Kalman. Desenvolveram um algoritmo iterativo computacionalmente simples que gera estimadores que dependem apenas dos resultados usuais do Filtro de Kalman ao longo do tempo. Definiram condições para que os estimadores obtidos pela maximização da verossimilhança fossem consistentes e assintoticamente normais. 


\section{Observações:}

1. Outros artigos tratam da estimação por maximização da função de verossimilhança como: de Jong (1988), de Jong e Chu-Chun-Lin (1994a) e Harvey e Peters (1990). Gupta e Mehra (1974) discutem aspectos numéricos da obtenção de estimadores de máxima verossimilhança pelo algoritmo Newton-Raphson e escore.

2. Segundo Shumway e Stoffer (1982), os métodos de máxima verossimilhança escore e Newton-Raphson apresentam problemas que são contornados utilizandose o algoritmo EM.

Os problemas apontados são: o Algoritmo Escore envolve, em cada iteração, cálculo da matriz inversa $I(\varphi)^{-1}$ que pode ter grande dimensão dependendo do número de parâmetros e a verossimilhança não necessariamente aumenta em passos sucessivos.

Segundo esses autores, as vantagens do algoritmo EM são o crescimento monótono da função de verossimilhança e a convergência garantida para pontos estacionários na família exponencial, além da simplicidade das equações do algoritmo.

Ainda segundo Shumway e Stoffer (1982), o algoritmo EM apresenta como desvantagem a convergência lenta obtida após muitas iterações e a não obtenção dos erros padrões estimados da forma convencional, dado que não é calculada a matriz das segundas derivadas parciais como nos outros métodos. Em Tanner (1996) encontramos uma discussão sobre como obter estes erros padrões.

3. Para os modelos ARMA, que podem ser representados na forma espaço de estados, a utilização do procedimento de estimação dos parâmetros por máxima verossimilhança e o cálculo do Filtro de Kalman são feitos segundo o procedimento acima. Ver Harvey e Phillips (1979). Para estender a utilização do Filtro de Kalman para os modelos ARIMA não estacionários, temos dificuldades na especificação das condições iniciais do filtro. Não se pode usar, como no caso estacionário, uma distribuição para o vetor de estado inicial porque no caso não estacionário essa distribuição não está definida. Vários trabalhos se dedicam a este problema de inicialização do Filtro de Kalman, como Kitagawa (1981), Bell e Hillmer (1991), Gómez e Maravall (1994), de Jong e Chu-Chun-Lin (1994a) e de Jong e Chu-Chun-Lin (1994b). 


\subsubsection{Métodos Bayesianos}

Os parâmetros dos modelos na forma de espaço de estados podem ser estimados utilizando-se a abordagem bayesiana. Deste modo são designados subjetivamente ou seqüencialmente via inferência bayesiana.

\section{i) Estimação de W:}

Para a estimação de W (matriz de covariâncias do vetor de estado $\theta_{t}$ ) na formulação bayesiana é utilizada a técnica de "fatores de desconto". A noção de descontos está associada à taxa de perda de informação no tempo, isto é, a perda de relevância das informações (observações) com o passar do tempo, significando que as informações mais recentes são mais relevantes no processo de modelagem. $\mathrm{O}$ fator de desconto é um número $\beta, 0<\beta \leq 1$ tal que quanto menor $\beta$ menos importância é dada às observações antigas, tornando desta forma o sistema mais adaptativo. Ver Souza (1989). Esta abordagem está descrita na seção 6.3 de West e Harrison (1997).

ii) Estimação de V:

No pacote bayesiano BATS (Bayesian Analysis of Time Series) está implementado um algoritmo relativo à "lei de variância" para a estimação seqüencial da variância observacional V. Este procedimento está apresentado na seção 6.3 e no apêndice III de Souza (1989).

iii) Estimação das matrizes F e G:

A estimação é realizada pela obtenção de distribuições a posteriori para os parâmetros em F e G utilizando-se prioris especificadas para os mesmos. Ver seção 9.3.3 em West e Harrison (1997) para mais detalhes.

\subsection{Modelos em Espaço de Estados Não-Normais e Não-Lineares}

Seja o seguinte modelo na forma de espaço de estados:

$$
\begin{aligned}
& y_{t}=h_{t}\left(\theta_{t}\right)+v_{t}, \\
& \theta_{t}=f_{t}\left(\theta_{t-1}\right)+w_{t},
\end{aligned}
$$


onde

$v_{t}$ e $w_{t}$ são não gaussianos;

$f_{t}(\cdot)$ e $h_{t}(\cdot)$ são funções não lineares que dependem de parâmetros desconhecidos.

Na forma geral acima, a utilização do Filtro de Kalman pode comprometer a obtenção de previsões ótimas, já que ele fornece neste caso apenas o melhor preditor linear.

Muitos trabalhos têm sido apresentados como sugestão para resolver esse modelo. O capítulo 6 de Kitagawa e Gersch (1996) traz um resumo das principais propostas e apresenta fórmulas recursivas para predição, filtragem e suavização deste modelo e também três abordagens computacionais alternativas: um método de integração numérica, um método para misturas de normais e um método de Monte Carlo. Em adição, a seção 6.6 apresenta uma derivação alternativa para o Filtro de Kalman.

Os artigos Carlin, Polson e Stoffer (1992) e Carter e Kohn (1994) apresentam soluções utilizando o amostrador de Gibbs. Este último tem como hipóteses modelos com erros misturas de normais e parâmetros variando no tempo.

O artigo Durbin e Koopman (1996) obtém aproximações para a função de verossimilhança para modelos na forma de espaço de estados não-normais e nãolineares a partir de simulações de Monte Carlo.

O artigo Fahrmeir (1992) trata de uma extensão do modelo não-linear e nãogaussiano. A abordagem é baseada na idéia de "posterior mode filtering" (filtragem da moda da distribuição a posteriori) para o modelo linear generalizado multivariado dinâmico. 


\section{Capítulo 3}

\section{Ondaletas}

\subsection{Introdução}

Segundo Meyer (1993), as ondaletas constituem novas bases para representar funções. É uma ferramenta matemática versátil, com boas propriedades e grande potencial de aplicação.

As ondaletas são localizadas no tempo (ou espaço) diferentemente das funções trigonométricas e sua utilização é apropriada para analisar sinais não estacionários, aproximando uma função através de combinação linear de ondaletas.

Atualmente as ondaletas são aplicadas a diversas áreas do conhecimento como processamento de sinais, codificação e compressão de imagens, música, etc. Em Estatística, são utilizadas na estimação de densidades, regressão não-paramétrica, estimação do espectro de processos estacionários e não-estacionários.

Mais informações sobre o assunto podem ser encontradas em Meyer (1993), Chui (1992), Hubbard (1996), Morettin (1997a), Morettin (1997b), Ogden (1997) e Vidakovic e Müller (1994).

\subsection{Ondaletas versus Análise de Fourier}

Como senos e cossenos na análise de Fourier, ondaletas são usadas como funções base para representar outras funções. Mas existe uma importante diferença entre as duas análises: as funções que formam a base na análise de Fourier são localizadas na freqüência enquanto as funções que formam a base de ondaletas são localizadas na escala (via dilatação) e no tempo (via translação). 
Além disso, as ondaletas representam uma grande classe de funções nas mais diversas formas. Por exemplo, funções descontínuas e com picos são melhor representadas por ondaletas do que por funções seno e cosseno.

Os coeficientes de ondaletas caracterizam o comportamento "local" de um sinal, ao passo que os coeficientes de Fourier caracterizam o comportamento "global" do sinal.

O artigo de Strang (1993) é uma boa introdução à comparação da transformada de Fourier com a transformada de ondaletas.

Seja $f$ uma função definida num espaço de funções de quadrado integrável no intervalo $[-\pi, \pi]$, isto é, $f \in L^{2}[-\pi, \pi]$. Na análise de Fourier, tais funções são geradas por uma superposição de exponenciais complexas $w_{n}(x)=\exp ($ inx $), \quad n=$ $0, \pm 1, \cdots$ obtidas por dilatações da função $w(x)=\exp (i x): w_{n}(x)=w(n x)$.

Isto significa que toda função $f \in L^{2}[-\pi, \pi]$ periódica pode ser escrita como

$$
f(t)=\sum_{n=-\infty}^{\infty} c_{n} \exp (\text { int })
$$

onde $c_{n}$ são os coeficientes de Fourier dados por

$$
c_{n}=\frac{1}{2 \pi} \int_{-\pi}^{\pi} f(t) \exp (-i n t) d t .
$$

A função $f$ pode ser expressa ainda como uma soma infinita de funções seno e cosseno dilatadas,

$$
f(x)=\frac{1}{2} a_{0}+\sum_{j=1}^{\infty}\left(a_{j} \cos (j x)+b_{j} \operatorname{sen}(j x)\right),
$$

para um conjunto apropriado de coeficientes $\left\{a_{0}, a_{1}, b_{1}, \cdots\right\}$.

Esta representação de Fourier da série é muito útil para representar qualquer função em termos de senos e cossenos porque o conjunto de funções $\{\operatorname{sen}(j \cdot), \cos (j \cdot)$, $j=1,2, \cdots\}$ juntamente com uma função constante formam uma base no espaço $L^{2}[-\pi, \pi]$.

Para se obter os coeficientes $\left\{a_{0}, a_{1}, b_{1}, \cdots\right\}$, fazemos o produto interno da função $f$ e a função base correspondente:

$$
a_{j}=\frac{1}{\pi}<f, \cos (j \cdot)>=\frac{1}{\pi} \int_{-\pi}^{\pi} f(x) \cdot \cos (j x) d x, \quad j=0,1,2, \cdots
$$




$$
b_{j}=\frac{1}{\pi}<f, \operatorname{sen}(j \cdot)>=\frac{1}{\pi} \int_{-\pi}^{\pi} f(x) \cdot \operatorname{sen}(j x) d x, \quad j=0,1,2, \cdots
$$

Os coeficientes $a_{j}$ e $b_{j}$ são medidas de "freqüência" da função $f$ no nível de resolução $j$.

O objetivo de se trabalhar com ondaletas é a extensão do fato de $f \in L^{2}[-\pi, \pi]$ para $f \in L^{2}(\mathbb{R})$ utilizando-se para isso uma única função $\psi$ para gerar este espaço. Isto é obtido por dilatações (ou compressões) e translações de $\psi$.

As funções que geram $L^{2}(\mathbb{R})$ devem decair rapidamente para zero, daí a idéia da utilização de pequenas ondas transladadas no tempo, as ondaletas.

$\mathrm{Na}$ próxima seção apresentamos as definições formais relacionadas com as ondaletas.

\subsection{Definições}

Definição 1: Seja $L^{2}(\mathbb{R})$ o espaço das funções $f$ mensuráveis de quadrado integrável sobre $\mathbb{R}$ isto é, $f$ definidas em $\mathbb{R}=(-\infty, \infty)$ satisfazendo:

$$
\int_{-\infty}^{\infty}|f(x)|^{2} d x<\infty
$$

As funções $f(x)$ devem decair para zero quando $|x| \rightarrow \infty$.

Partimos de uma ondaleta $\psi$, que gera $L^{2}(\mathbb{R})$ a partir de dilatações (compressões) e translações de modo a cobrir $\mathbb{R}$, ou seja, consideramos ondaletas

$$
\psi_{a, b}(t)=|a|^{1 / 2} \cdot \psi\left(\frac{t-b}{a}\right), a>0,-\infty<b<\infty .
$$

A função $\psi$ é chamada ondaleta-mãe.

Uma particularização da equação (3.1) pode ser obtida de $\psi(t)$ por uma dilatação binária $2^{j}$ e uma translação diádica $k 2^{-j}$,

$$
\psi_{j, k}(t)=\psi\left(2^{j} t-k\right) \quad j, k \in Z \text {. }
$$

O fator de escala $2^{-j}$ é chamado de fator de dilatação e $j$ é o nível associado à escala $2^{-j}$. O parâmetro $k 2^{-j}$ é chamado de parâmetro de localização e $k$ é o índice de translação associado a $k 2^{-j}$. 


\section{Definição 2:}

i) Produto interno entre $f$ e $g:\langle f, g\rangle=\int f(x) \cdot g(x) d x$;

ii) Norma de $f$ : $\|f\|=\sqrt{\langle f, f\rangle}=\sqrt{\int f(x)^{2} d x}$;

iii) Duas funções $f_{1}$ e $f_{2} \in L^{2}[a, b]$ são ditas ortogonais se $\left\langle f_{1}, f_{2}\right\rangle=0$;

iv) Uma seqüência de funções $\left\{f_{j}\right\}$ é dita ser ortonormal se cada par $f_{i}, f_{j}$ for ortogonal, $\forall i \neq j$, e $\left\|f_{j}\right\|=1, \forall j$.

As funções $\left\{\psi_{j, k}, j, k \in Z\right\}$ formam uma base não necessariamente ortogonal. A vantagem de se trabalhar com bases ortogonais é permitir a reconstrução perfeita do sinal original a partir dos coeficientes da transformada. Meyer provou que é possível construir tais bases.

Suponha

$$
\psi_{j, k}(t)=2^{j / 2} \psi\left(2^{j} t-k\right) \quad j, k \in Z
$$

uma base ortonormal gerada por $\psi$, tal que para qualquer $f \in L^{2}(\mathbb{R})$,

$$
f(t)=\sum_{j=-\infty}^{\infty} \sum_{k=-\infty}^{\infty} c_{j, k} \cdot \psi_{j, k}(t)
$$

onde $c_{j, k}=\int_{-\infty}^{\infty} f(t) \cdot \psi_{j, k}(t) d t$ são os coeficientes de ondaletas. Então estas ondaletas satisfazem a certas propriedades, algumas das quais são:

i) $\int_{-\infty}^{\infty} \psi(x) d x=0$;

ii) os primeiros $(r-1)$ momentos de $\psi$ são nulos, isto é , $\int_{-\infty}^{\infty} t^{j} \psi(t) d t=0, j=0,1, \cdots, r-1, r \geq 1 ;$ $\psi$.

iii) $\int_{-\infty}^{\infty}\left|t^{r} \psi(t)\right| d t<\infty$, onde $\mathrm{r}$ está relacionado com o grau de suavidade de

Ver também a seção 3.4 .

Definição 3: Uma função $\phi$ é chamada função escala ou ondaleta-pai se é definida como solução da seguinte equação: 


$$
\phi(t)=\sqrt{2} \sum_{k} l_{k} \cdot \phi(2 t-k) .
$$

Esta função gera uma família ortonormal em $L^{2}(\mathbb{R})$,

$$
\phi_{j, k}(t)=2^{j / 2} \phi\left(2^{j} t-k\right), j, k \in Z,
$$

que satisfaz as propriedades i), ii) e iii) acima e é ortogonal a todas as translações de $\phi$.

Pode-se obter $\psi$ a partir de $\phi$ através da equação:

$$
\psi(t)=\sqrt{2} \sum_{k} h_{k} \cdot \phi(2 t-k),
$$

onde $h_{k}=(-1)^{k} \cdot l_{1-k}$ é chamada "quadrature mirror filter relation" e $l_{k}$ e $h_{k}$ são os coeficientes de filtros passa-baixo e passa-alto, respectivamente, usados para calcular a transformada de ondaleta discreta. Estes coeficientes são dados por

$$
\begin{aligned}
& l_{k}=\sqrt{2} \int_{-\infty}^{\infty} \phi(t) \phi(2 t-k) d t \mathrm{e} \\
& h_{k}=\sqrt{2} \int_{-\infty}^{\infty} \psi(t) \phi(2 t-k) d t .
\end{aligned}
$$

As equações (3.5) e (3.7) são chamadas equações de dilatação.

\subsection{Propriedades}

Destacamos algumas propriedades das ondaletas segundo Jawerth e Sweldens (1994).

a) Ortogonalidade: é uma propriedade conveniente para muitas situações.

b) Suporte compacto ou decaimento suficientemente rápido no tempo: relacionado com a habilidade das ondaletas de localizar características de um sinal no tempo e no espaço.

c) Suavização: funções suaves são desejadas em aplicações em análises numéricas onde são envolvidas derivadas. Uma medida de suavização é dada pelo número de derivadas da ondaleta. O número de momentos nulos também está 
relacionado com a suavização (quanto maior o número de momentos nulos, mais suave é a ondaleta).

d) Expressão analítica: nem sempre se tem a expressão analítica de uma ondaleta. Em particular, as ondaletas Haar, Chapéu Mexicano, Morlet e Shannon possuem expressão analítica.

e) Simetria: importante em aplicações em processamento de sinais.

f) Momentos nulos: importante para detectar singularidades e caracterização de espaços suavizados. Quanto maior o número de momentos nulos de uma dada ondaleta, mais suave ela é.

\subsection{Encolhimento de Ondaletas}

O encolhimento de ondaletas ("wavelets shrinkage") é um procedimento que tem como objetivo a redução ou remoção do ruído presente num sinal. Isto é feito reduzindo ou anulando a magnitude dos coeficientes das ondaletas.

O encolhimento de ondaletas é aplicado a estimadores dos coeficientes obtidos pela transformada discreta de ondaletas de uma dada função de tal forma que os coeficientes pequenos são truncados (isto significa anular coeficientes menores do que determinado valor). Em seguida, aplica-se a transformada inversa e obtém-se uma estimativa da função cujos coeficientes na representação por ondaletas são desprovidos de ruídos.

A idéia do encolhimento de ondaletas é incluir coeficientes "grandes" em relação a um valor limiar.

Seja $f \in L^{2}(\mathbb{R})$. Para um dado valor $\lambda$ limiar, um estimador $\hat{f}$ de $f$ pode ser escrito como

$$
\hat{f}_{\lambda}(u)=\sum_{j=0}^{J-1} \sum_{k=0}^{2^{j}-1} I\left(\left|w_{j, k}^{(n)}\right|>\lambda\right) \cdot w_{j, k}^{(n)} \cdot \psi_{j, k}(u),
$$

onde $I(A)$ é a função indicadora do conjunto $A, w_{j, k}^{(n)}$ são os coeficientes das ondaletas $\psi_{j, k}(u)$ e $n$ é número total de dados.

Isto representa "manter ou matar" a ondaleta onde os coeficientes maiores que determinado limiar são mantidos e os demais zerados. Essa limiarização pode ser pensada como um operador não linear aplicado ao vetor de coeficientes, resultando num novo vetor de coeficientes estimados. 
Para tomar a decisão de qual coeficiente se mantém ou torna-se zero, leva-se em conta dois fatores que afetam a precisão dos estimadores:

i) o tamanho $n$ da amostra e

ii) o nível do ruído $\sigma^{2}$.

Deste modo, deve-se incluir o coeficiente em amostras grandes e/ou nível de ruído baixo.

O encolhimento de ondaletas é capaz de remover os ruídos de um sinal enquanto preserva as características desse sinal.

A estimação usando encolhimento de ondaletas se baseia em três princípios:

1. as características de um sinal podem ser representadas por poucos coeficientes de ondaletas;

2. os ruídos afetam todos os coeficientes de ondaletas;

3. truncando os coeficientes de ondaletas que estão tendendo a zero, os ruídos podem ser removidos e as características do sinal são preservadas.

Referências importantes para o encolhimento de ondaletas são encontradas em Donoho, Johnstone, Kerkyacharian e Picard (1995), Donoho e Johnstone (1995) e Donoho, Johnstone, Kerkyacharian e Picard (1996).

O procedimento de encolhimento de ondaletas pode ser dividido em duas etapas:

i) escolher o tipo de suavização, que pode ser:

- dura ("hard shrinkage"): é uma função descontínua do tipo "mata ou preserva".

$$
\delta_{\lambda}^{H}(x)= \begin{cases}0, & -\lambda \leq x \leq \lambda \\ x, & x>\lambda \text { ou } x<-\lambda\end{cases}
$$

- suave ("soft shrinkage"): é uma função contínua do tipo "mata ou encolhe".

$$
\delta_{\lambda}^{S}(x)= \begin{cases}0, & -\lambda \leq x \leq \lambda \\ x-\lambda, & x>\lambda \\ x+\lambda, & x<-\lambda\end{cases}
$$

ii) escolher os parâmetros $\lambda$ e $\sigma$ : 
- $\lambda$ pode ser estimado por vários procedimentos, por exemplo, pelo limiar universal (Donoho et al., 1995)

$$
\begin{gathered}
\lambda=\sigma \cdot \sqrt{2 \log (n),}, \text { ou } \\
\lambda_{j}=\sigma_{j} \cdot \sqrt{2 \log \left(n_{j}\right)},
\end{gathered}
$$

onde

$n$ é o tamanho da série;

$n_{j}$ é o total de coeficientes de ondaletas para o nível $j$.

- $\sigma\left(\sigma_{j}\right)$ pode ser estimado considerando-se o desvio padrão de todos os coeficientes de ondaletas (todos os coeficientes no nível $j$ ). Métodos alternativos são encontrados em Donoho et al. (1995).

\subsection{Principais Ondaletas}

Existem muitos tipos de ondaletas das mais diversas formas. Ondaletas podem ser suaves ou não, com suporte compacto ou não, com expressões matemáticas simples ou não. Apresentamos abaixo algumas ondaletas separadas em dois grupos: as que têm expressão analítica e as que não têm.

\section{i) Ondaletas com expressão analítica:}

- Haar: A mais antiga e mais simples de todas, a função Haar data de 1910 e tem sido utilizada em muitos campos da Matemática. Dilatações e translações da função $\psi$ definem uma base ortogonal em $L^{2}(\mathbb{R})$ :

$$
\begin{gathered}
\psi(t)=\left\{\begin{array}{cl}
1, & 0 \leq t<1 / 2 \\
-1, & 1 / 2 \leq t<1 \\
0, & \text { caso contrário, }
\end{array}\right. \\
\psi_{j, k}(t)=\left\{\begin{array}{cl}
2^{j / 2}, & 2^{-j} k \leq t<2^{-j}(k+1 / 2) \\
-2^{j / 2}, & 2^{-j}(k+1 / 2) \leq t<2^{-j}(k+1) \\
0, & \text { caso contrário, }
\end{array}\right. \\
j=0,1, \cdots, m-1, \quad k=0,1, \cdots, 2^{j}-1 .
\end{gathered}
$$


- Chapéu Mexicano: Obtida a partir da segunda derivada da curva gaussiana:

$$
\begin{gathered}
\nu \cdot(t)=\left(1-t^{2}\right) \cdot \exp \left(-t^{2} / 2\right) \text { e } \\
\psi_{j, k}(t)=2^{j / 2} \cdot\left(1-\left(2^{j} t-k\right)^{2}\right) \cdot \exp \left(-\left(2^{j} t-k\right)^{2} / 2\right) .
\end{gathered}
$$

- Morlet ou Gaussiana Modulada: Função complexa para algum $w_{0}$ fixo:

$$
\psi(t)=\exp \left(i w_{0} t\right) \cdot \exp \left(-t^{2} / 2\right) .
$$

Parte real:

$$
\begin{gathered}
\psi_{\text {real }}(t)=\cos \left(w_{0} t\right) \cdot \exp \left(-t^{2} / 2\right) \text { e } \\
\psi_{j, k}(t)=2^{j / 2} \cdot \cos \left(w_{0}\left(2^{j} t-k\right)\right) \cdot \exp \left(-\left(2^{j} t-k\right)^{2} / 2\right) .
\end{gathered}
$$

- Ondaleta Shannon:

$$
\begin{gathered}
\psi(t)=\frac{\operatorname{sen}\left(\frac{\pi t}{2}\right) \cdot \cos \left(\frac{3 \pi t}{2}\right)}{\frac{\pi t}{2}} \\
\psi_{j, k}(t)=\frac{2^{j / 2} \cdot \operatorname{sen}\left(\frac{\pi\left(2^{j} t-k\right)}{2}\right) \cdot \cos \left(\frac{3 \pi\left(2^{j} t-k\right)}{2}\right)}{\frac{\pi\left(2^{j} t-k\right)}{2}} .
\end{gathered}
$$

\section{ii) Ondaletas sem expressão analítica:}

- Daublets, Symmlets e Coiflets são ondaletas construídas por Ingrid Daubechies. Têm suporte compacto e a última possui como propriedade momentos nulos para $\phi$ e $\psi$. Ver Daubechies (1992).

Apresentamos a seguir gráficos das ondaletas com expressão analítica. 

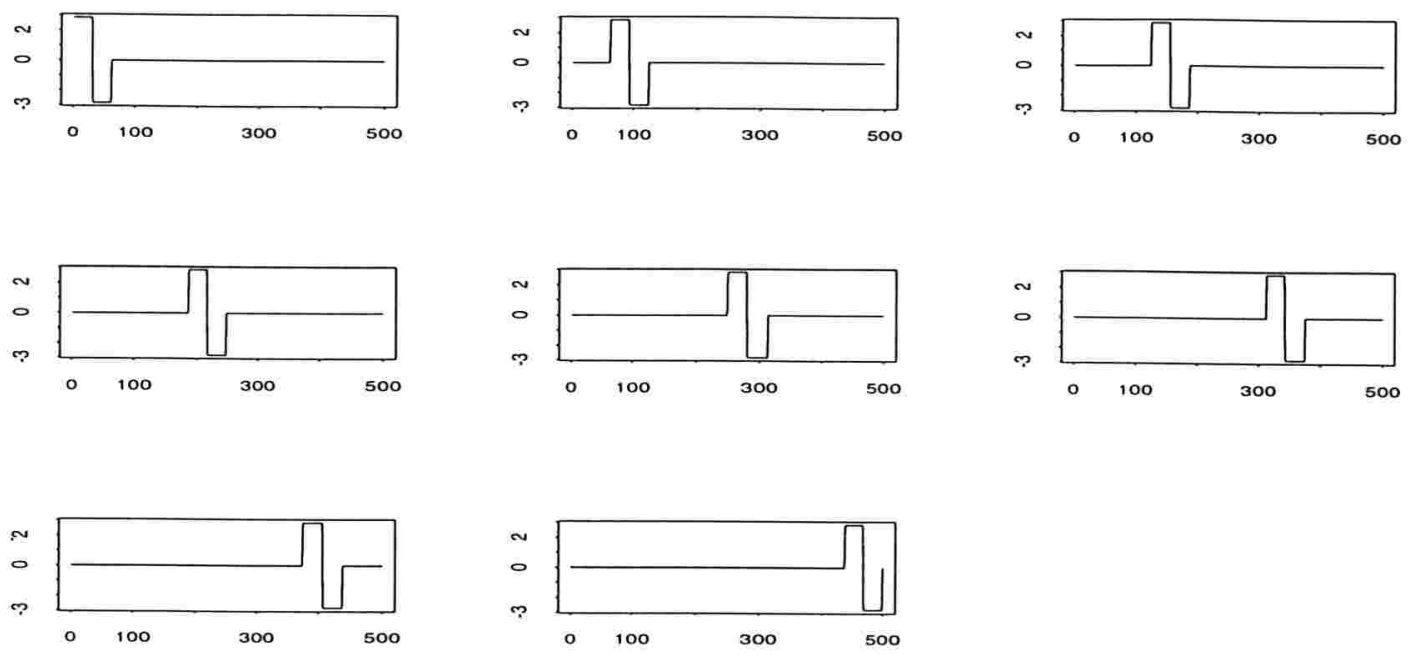

Figura 3.1: Gráficos da Ondaleta Haar para $j=4$ e $k=1, \ldots, 8$.
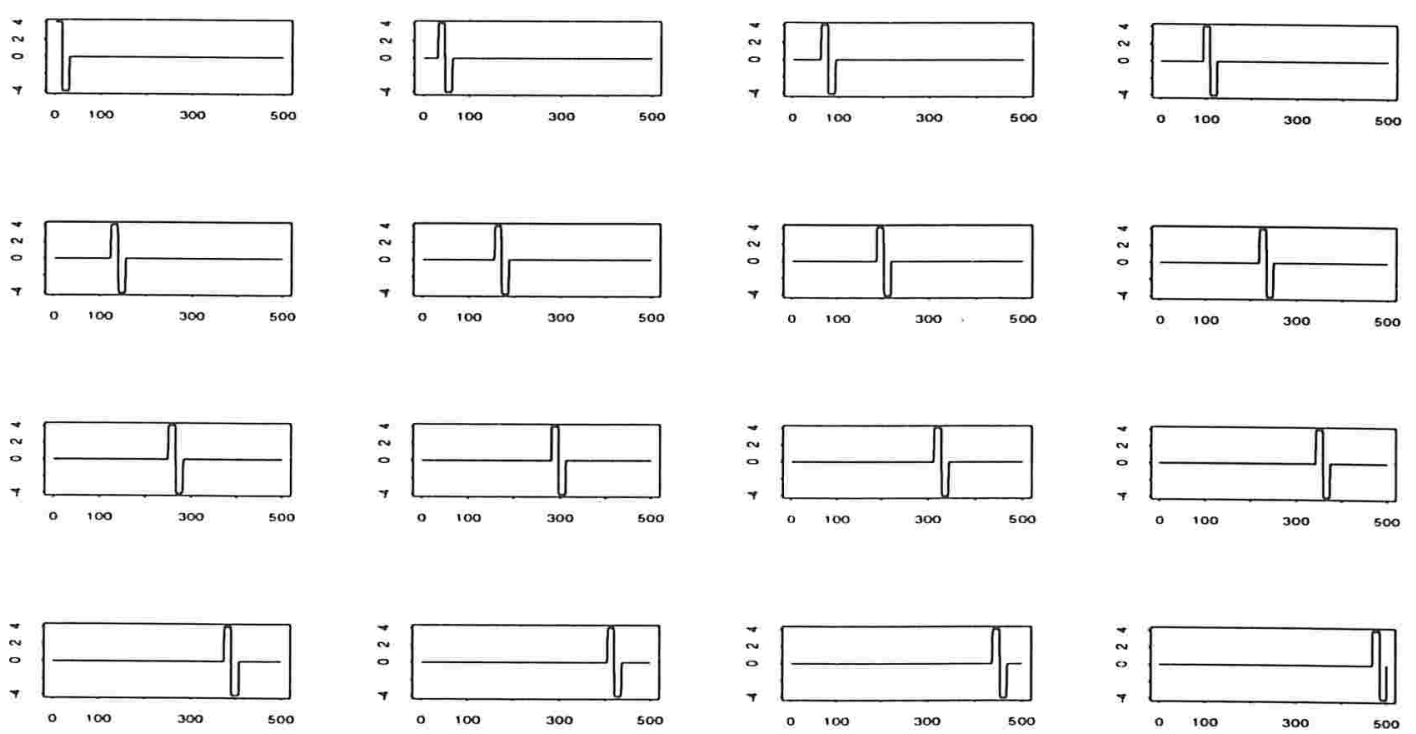

Figura 3.2: Gráficos da Ondaleta Haar para $j=5$ e $k=1, \ldots, 16$. 

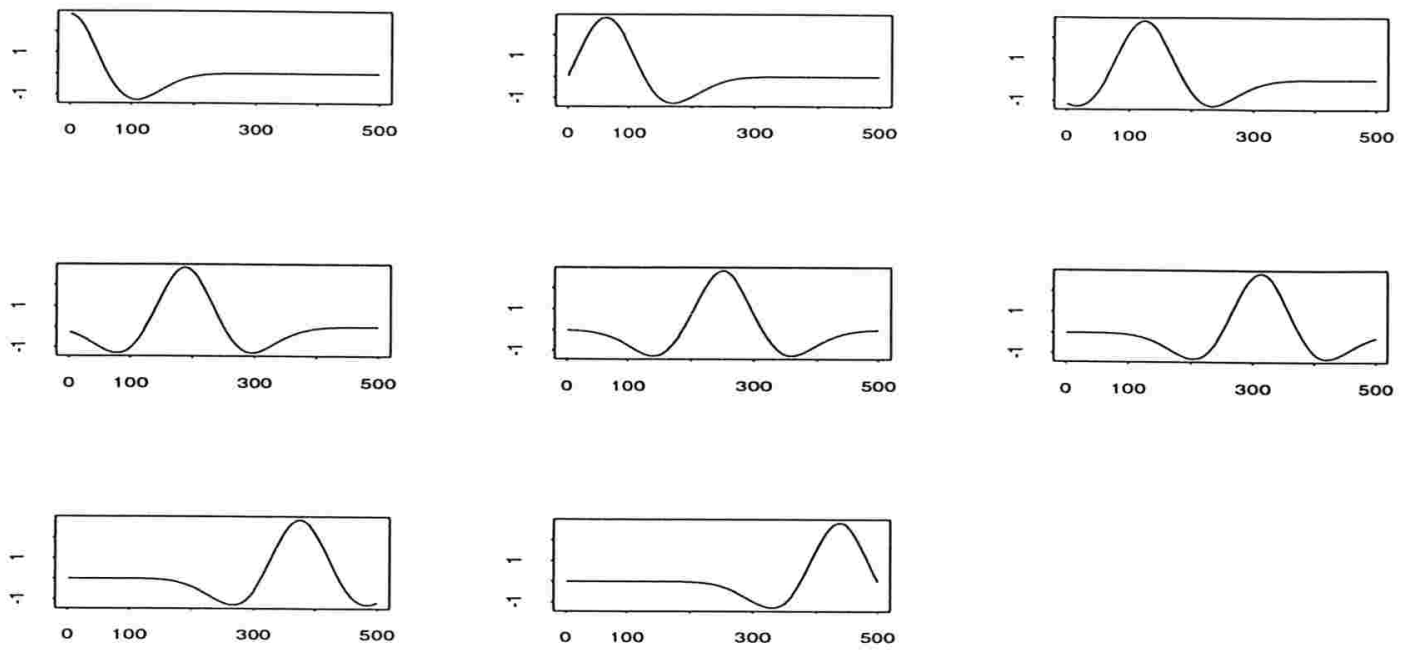

Figura 3.3: Gráficos da Ondaleta Chapéu Mexicano para $j=4$ e $k=1, \ldots, 8$.
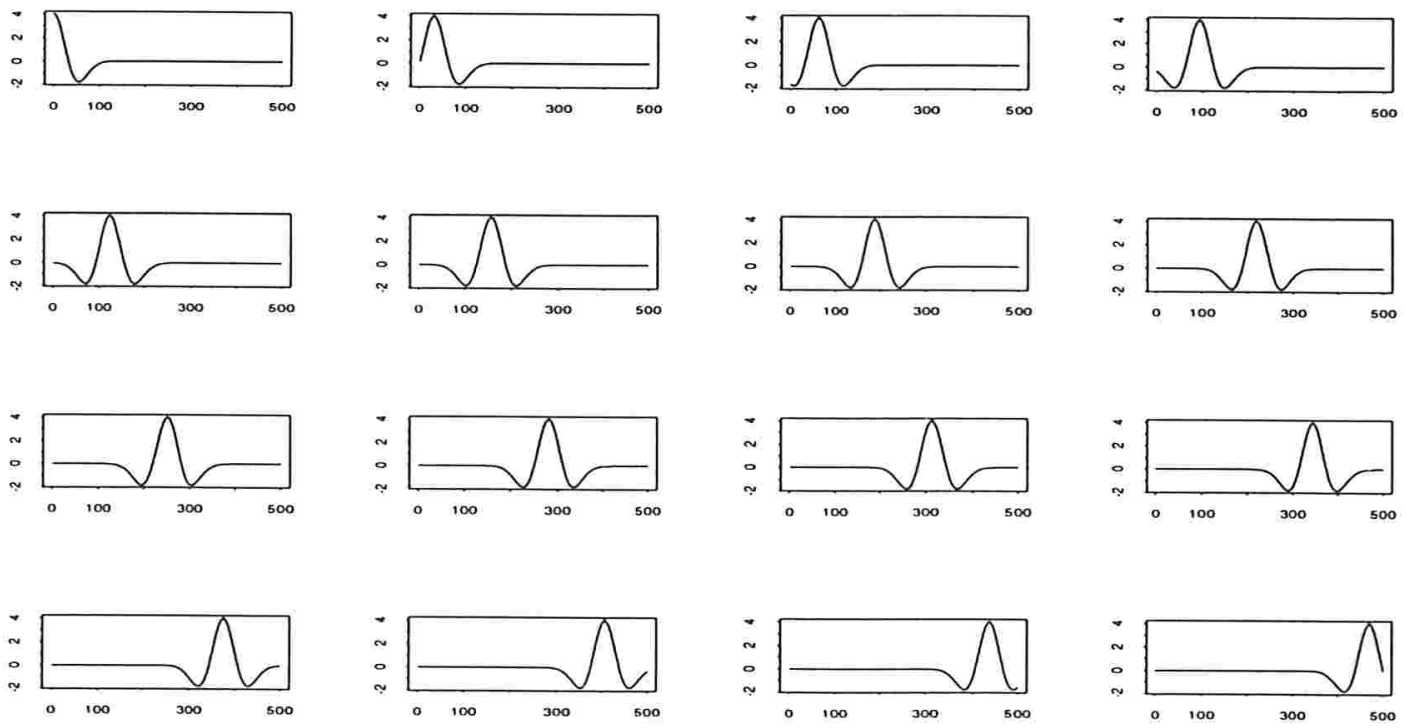

Figura 3.4: Gráficos da Ondaleta Chapéu Mexicano para $j=5$ e $k=1, \ldots, 16$. 

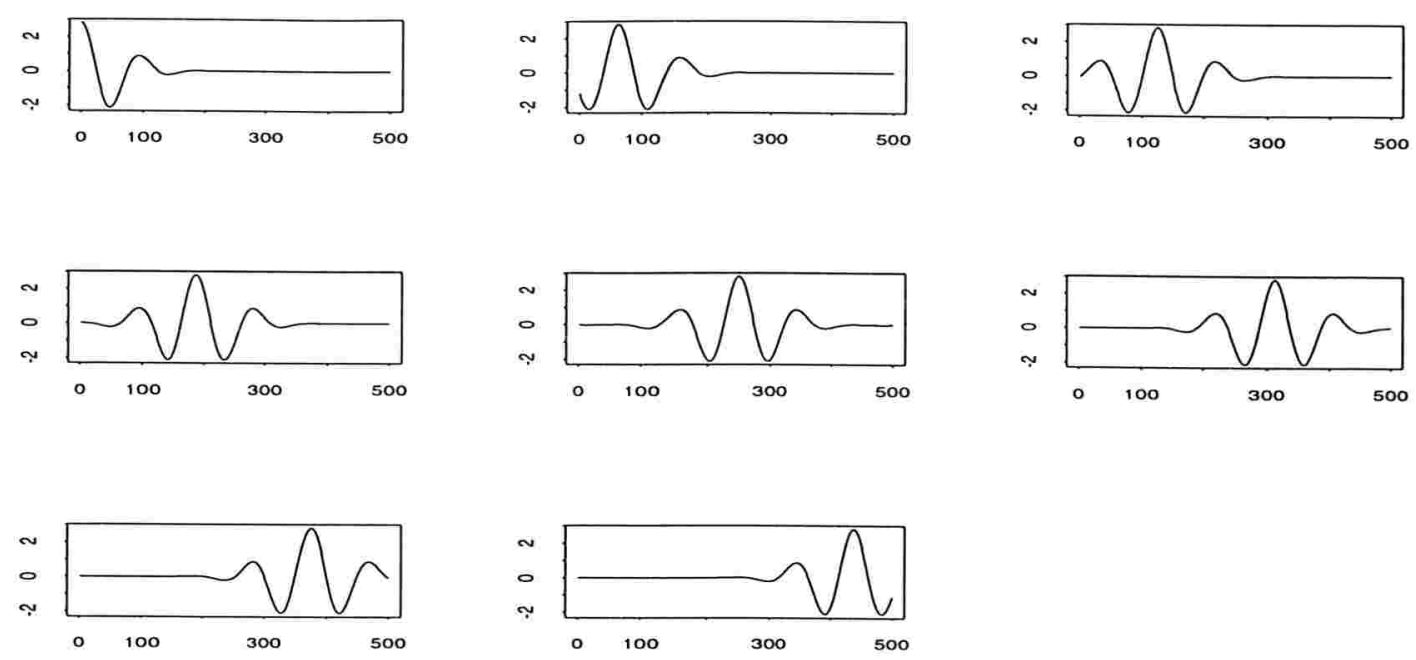

Figura 3.5: Gráficos da Ondaleta Morlet para $j=4$ e $k=1, \ldots, 8$.
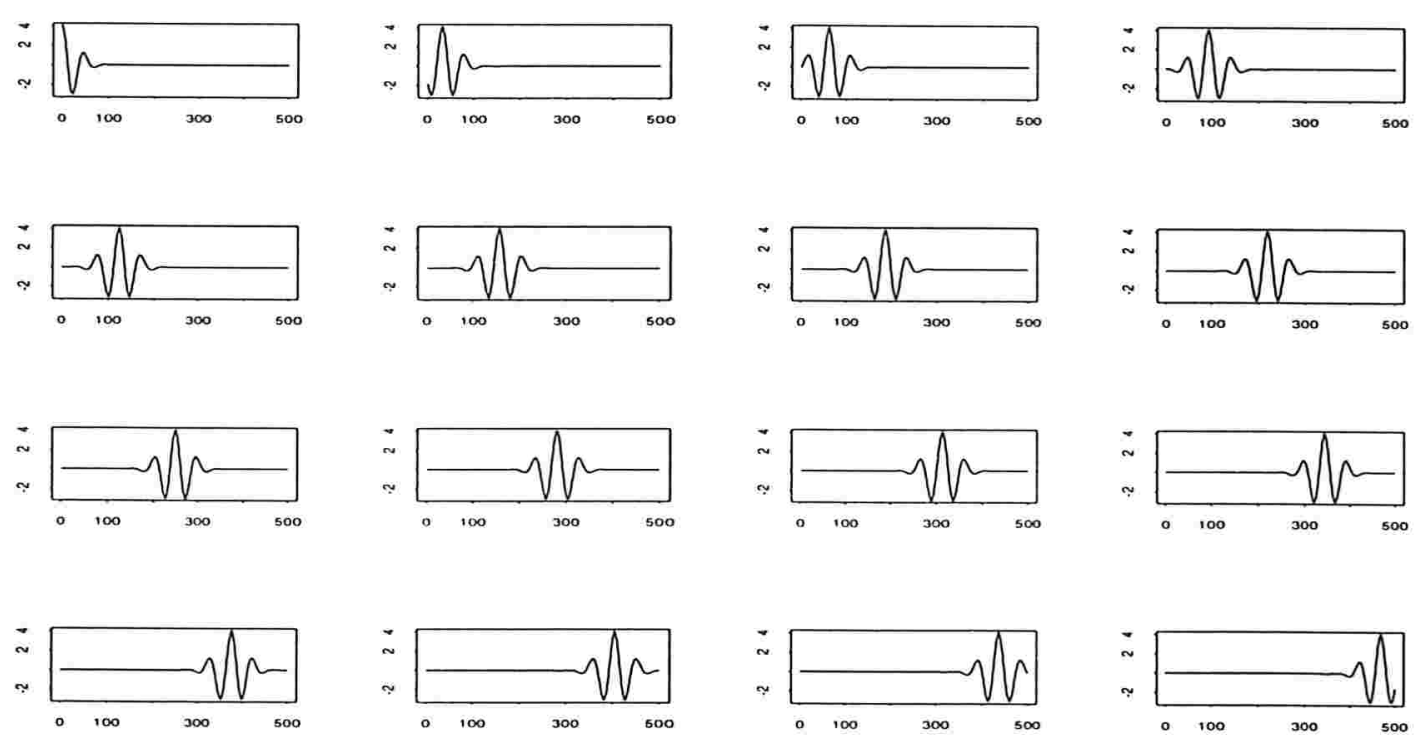

Figura 3.6: Gráficos da Ondaleta Morlet para $j=5$ e $k=1, \ldots, 16$. 

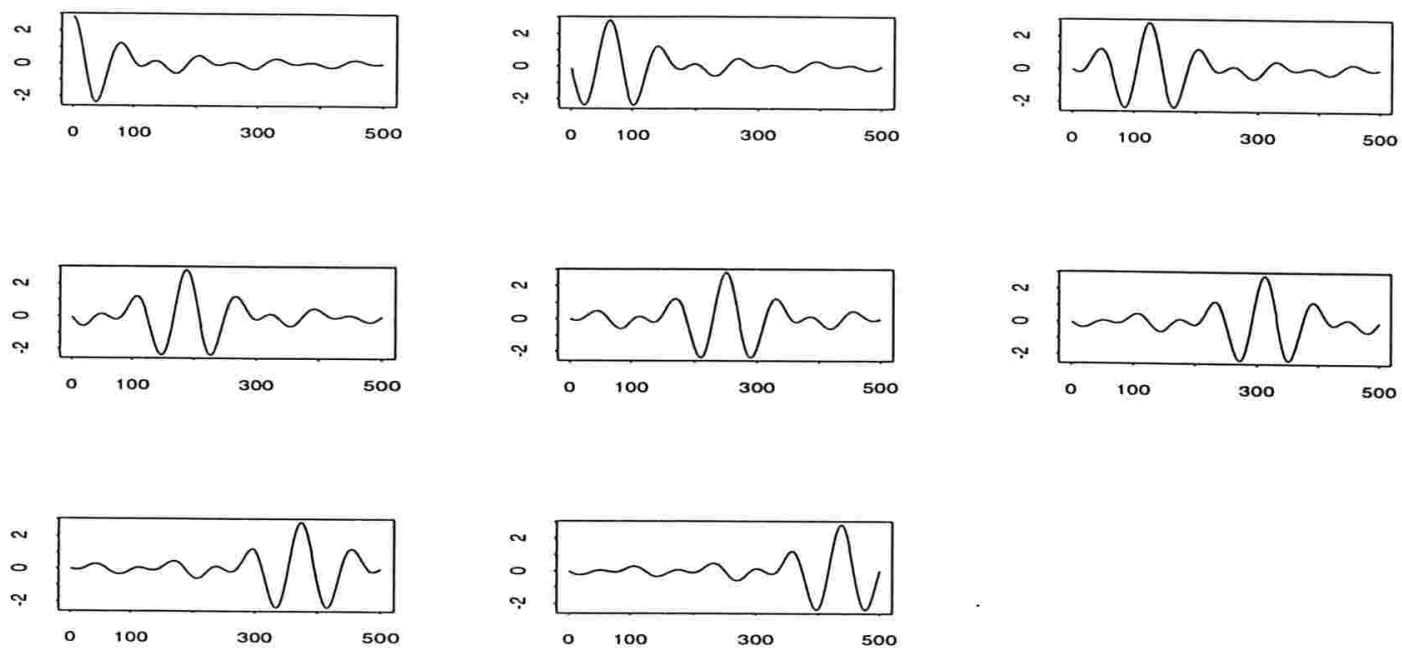

Figura 3.7: Gráficos da Ondaleta Shannon para $j=4$ e $k=1, \ldots, 8$.
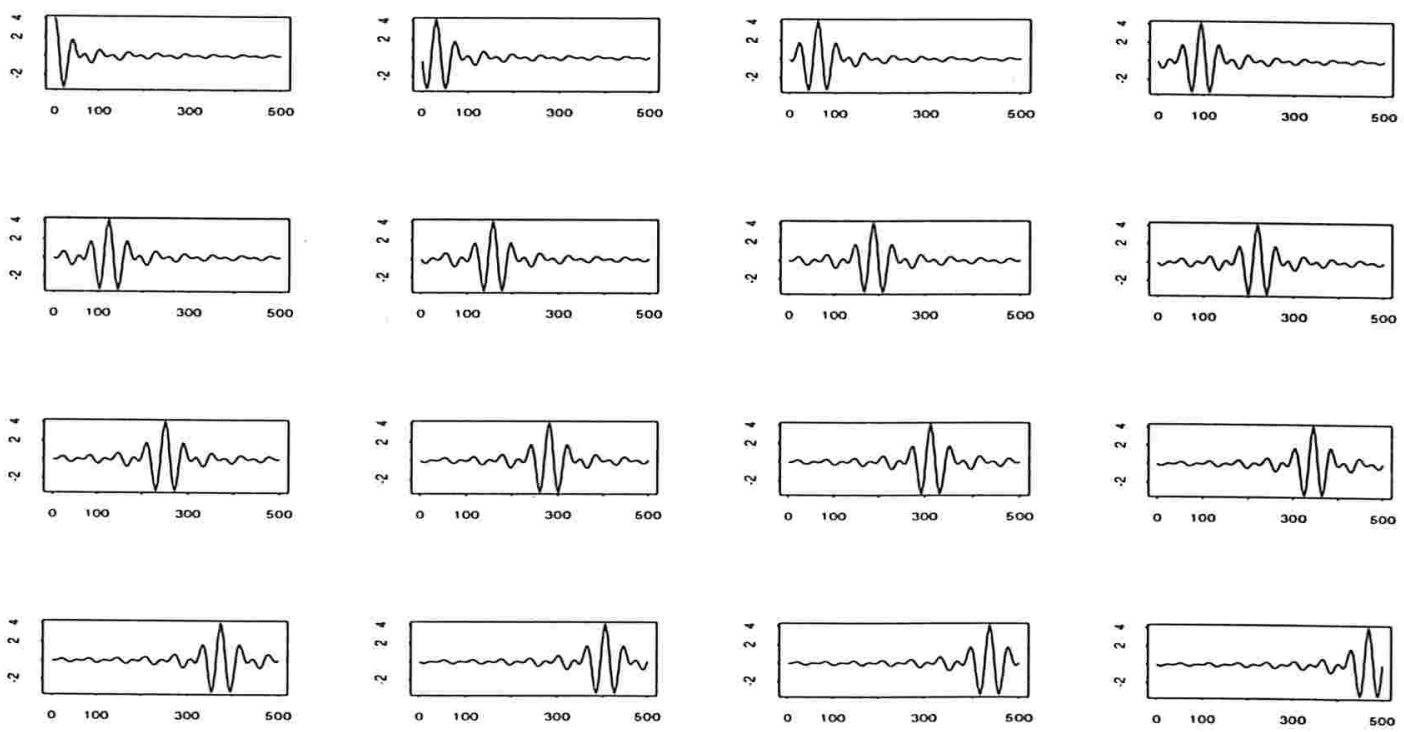

Figura 3.8: Gráficos da Ondaleta Shannon para $j=5$ e $k=1, \ldots, 16$. 


\section{Capítulo 4}

\section{Modelos com Ondaletas na Matriz de Transição}

\subsection{Introdução}

No contexto estatístico, a forma em espaço de estados é empregada para representar uma grande quantidade de estruturas. Algumas dessas estruturas apresentam parâmetros a serem estimados (hiperparâmetros na designação de Harvey (1990)) na matriz de transição, $G_{t}$. A idéia de se utilizar ondaletas em $G_{t}$ é incluir uma variação temporal na matriz de parâmetros.

Os modelos estruturais (ver Capítulo 2) que representam a série temporal através de componentes básicas de tendência, sazonalidade, componente cíclica e ruído aleatório, quando expressos na forma de espaço de estados apresentam de forma geral (dependendo da presença das componentes) parâmetros em $G_{t}$.

Neste caso, os parâmetros em $G_{t}$ não dependem do tempo e toda dependência temporal é alocada no vetor de estado $\theta_{t}$.

Vejamos o exemplo do modelo estrutural tendência mais ciclo. Seja o seguinte modelo estrutural:

$$
y_{t}=\mu_{t}+\varphi_{t}+\varepsilon_{t}
$$

onde

$$
\mu_{t}=\mu_{t-1}+\beta_{t-1}+\delta_{\mu_{t}}
$$


$\beta_{t-1}$ é a taxa de crescimento do processo que ocorreu no instante $t-1$;

$\delta_{\mu_{t}}$ é a perturbação aleatória do nível no instante $t$, supostamente com as seguintes propriedades:

$\delta_{\mu_{t}} \sim N\left(0, V_{\mu}\right) ;$

$E\left(\delta_{\mu_{i}} \cdot \delta_{\mu_{j}}\right)=0, \quad i \neq j \quad$ e $i, j=1,2, \cdots, n$;

$\left(\begin{array}{c}\varphi_{t} \\ \varphi_{t}^{*}\end{array}\right)=\rho\left(\begin{array}{cc}\cos \lambda & \operatorname{sen} \lambda \\ -\operatorname{sen} \lambda & \cos \lambda\end{array}\right)\left(\begin{array}{c}\varphi_{t-1} \\ \varphi_{t-1}^{*}\end{array}\right)+\left(\begin{array}{c}w_{c t} \\ w_{c t}^{*}\end{array}\right)$

$\varphi_{t}^{*}$ é a componente cíclica associada;

$w_{c t}^{*}$ é a perturbação correspondente com as mesmas características de $w_{c t}$.

Então temos:

$$
\begin{gathered}
F_{t}=\left[\begin{array}{ccccc}
1 & 0 & \vdots & 1 & 0
\end{array}\right]^{\prime}, \\
G_{t}=\left(\begin{array}{ccccc}
1 & 1 & \vdots & 0 & 0 \\
0 & 1 & \vdots & 0 & 0 \\
\cdots & \cdots & \cdots & \cdots & \cdots \\
0 & 0 & \vdots & \rho \cos \lambda & \rho \operatorname{sen} \lambda \\
0 & 0 & \vdots & -\rho \operatorname{sen} \lambda & \rho \cos \lambda
\end{array}\right), \\
W_{t}=\left(\begin{array}{ccccc}
V_{\mu} & 0 & \vdots & 0 & 0 \\
0 & V_{\beta} & \vdots & 0 & 0 \\
\cdots & \cdots & \cdots & \cdots & \cdots \\
0 & 0 & \vdots & V_{w_{c}} & 0 \\
0 & 0 & \vdots & 0 & V_{w_{c}}
\end{array}\right) \mathrm{e} \\
\theta_{t}=\left[\begin{array}{lllll}
\mu_{t} & \beta_{t} & \vdots & \varphi_{t} & \varphi_{t}^{*}
\end{array}\right] .
\end{gathered}
$$


Para estes modelos, a parametrização da matriz $G_{t}$ por ondaletas não é apropriada porque a mesma não varia ao longo do tempo.

Uma possível aplicação prática do uso de ondaletas é nos modelos na forma de espaço de estados que representam os modelos $A R M A(p, q)$ com parâmetros variando no tempo. Seja o modelo ARMA com os parâmetros variando no tempo:

$$
\sum_{j=0}^{p} a_{j}\left(\frac{t}{T}\right)\left(X_{t-j, T}-\mu\left(\frac{t-j}{T}\right)\right)=\sum_{j=0}^{q} b_{j}\left(\frac{t}{T}\right) \sigma\left(\frac{t-j}{T}\right) \varepsilon_{t-j}, \quad \varepsilon_{t} \text { iid }(0,1) .
$$

Podemos representar o modelo acima na forma de espaço de estados:

$$
\begin{aligned}
& y_{t}=F_{t} \theta_{t}+\mu\left(\frac{t}{T}\right) \mathrm{e} \\
& \theta_{t}=G_{t} \theta_{t-1}+R_{t, T} \varepsilon_{t},
\end{aligned}
$$

onde

$$
\begin{aligned}
& F_{t}=\left(\begin{array}{llll}
1 & 0 & 0 & \cdots
\end{array}\right), \\
& G_{t}=\left(\begin{array}{cccccccc}
-a_{1}\left(\frac{t}{T}\right) & \ldots & \ldots & -a_{p}\left(\frac{t}{T}\right) & b_{1}\left(\frac{t}{T}\right) & \ldots & \ldots & b_{q}\left(\frac{t}{T}\right) \\
1 & 0 & \ldots & 0 & 0 & \ldots & \ldots & 0 \\
\vdots & \vdots & \vdots & \vdots & \vdots & \vdots & \vdots & \vdots \\
0 & \ldots & 1 & 0 & 0 & \ldots & \ldots & 0 \\
0 & \ldots & \ldots & 0 & 1 & \ldots & 0 & 0 \\
\vdots & \vdots & \vdots & \vdots & \vdots & \vdots & \vdots & \vdots \\
0 & \ldots & \ldots & 0 & 0 & \ldots & 1 & 0
\end{array}\right), \\
& \theta_{t}=\left(X_{t, T}-\mu\left(\frac{t}{T}\right), \cdots, X_{t-p+1, T}-\mu\left(\frac{t-p+1}{T}\right), \sigma\left(\frac{t}{T}\right) \varepsilon_{t}, \cdots, \sigma\left(\frac{t-q+1}{T}\right) \varepsilon_{t-q+1}\right)^{\prime} \mathrm{e} \\
& R_{t, T}=\left(\sigma\left(\frac{t}{T}\right), \ldots, 0, \sigma\left(\frac{t}{T}\right), \ldots, 0\right)^{\prime} .
\end{aligned}
$$


Neste caso, a matriz $G_{t}$ é composta pelos parâmetros autoregressivos $a_{i}(t / T)$, $i=1, \cdots, p$ e os parâmetros de médias móveis $b_{j}(t / T), j=1, \cdots, q$ e podemos utilizar as ondaletas para a estimação dos parâmetros em $G_{t}$.

Dahlhaus, em uma série de trabalhos abordou este problema de estimação de parâmetros variáveis ao longo do tempo a partir da teoria de modelos localmente estacionários. A definição de modelos localmente estacionários e suas propriedades foram desenvolvidas por ele devido à dificuldade de se formular uma teoria assintótica para modelos não estacionários.

Segundo Dahlhaus (1997b), os modelos AR, MA e ARMA com parâmetros variando no tempo são localmente estacionários. Destacamos a seguir alguns dos seus resultados:

1) Obteve propriedades assintóticas para estimadores de máxima verossimilhança de parâmetros de processos localmente estacionários e representou em espaços de estado o modelo ARMA. Dahlhaus (1993b) e capítulo 7 de Dahlhaus (1997b).

2) Formulou uma modelagem paramétrica para os processos localmente estacionários e obteve estimadores por quase-verossimilhança, fazendo um estudo de simulação de um modelo AR(2) com parâmetros variando no tempo. Dahlhaus (1993a), Dahlhaus (1997a) e capítulo 5 de Dahlhaus (1997b).

3) Determinou estimadores de mínimos quadrados para um processo autoregressivo com parâmetros variando no tempo utilizando ondaletas e obteve propriedades dos estimadores. Dahlhaus, Neumann e von Sachs (1997).

Nas próximas seções apresentamos a formulação do modelo com ondaletas e as estimativas dos parâmetros.

\subsection{Formulação do Modelo}

Seja o seguinte modelo na forma de espaço de estados:

$$
\begin{aligned}
y_{t} & =F_{t} \theta_{t}+v_{t}, \\
\theta_{t} & =G_{t} \theta_{t-1}+w_{t} \mathrm{e} \\
G_{t}^{(l, m)} & =\sum_{j, k} \beta_{j k}^{(l, m)} \psi_{j, k}(t / T),
\end{aligned}
$$


onde

$y_{t}$ é o vetor de observações $(r \times 1), t=1, \ldots, n$;

$F_{t}$ é a matriz do sistema $(r \times p)$;

$\theta_{t}$ é o vetor de estados $(p \times 1)$;

$G_{t}$ é a matriz de transição $(p \times p)$;

$(l, m)$ denota o elemento $(l, m)$ da matriz $G_{t}$;

$\beta_{j k}$ são parâmetros associados às ondaletas da matriz $G_{t}$;

$\psi_{j, k}(t / T)$ é a ondaleta no tempo $(t / T)$, fator de escala j e fator de translação $\mathrm{k}, \operatorname{com} T=n$.

$v_{t}$ é o vetor de erros associado às observações $(r \times 1)$;

$w_{t}$ é o vetor de erros associado ao estado $(p \times 1)$.

Sejam as seguintes hipóteses associadas ao modelo:

i) $v_{t} \sim$ iid $N\left(0, V_{t}\right)$;

ii) $w_{t} \sim$ iid $N\left(0, W_{t}\right)$;

iii) $v_{t}$ independente de $w_{t}$;

iv) $\left(\theta_{0} \mid D_{0}\right) \sim N(\mu, \Sigma)$, onde $D_{0}$ é o conjunto de informações no tempo $t=0$.

Queremos estimar o vetor de estado $\theta_{t}$ e o vetor de parâmetros $\varphi=\left(F_{t}, \beta_{j k}, V_{t}, W_{t}\right)$.

\subsection{Estimação dos Parâmetros}

A estimação dos parâmetros para o modelo apresentado em (4.2) será feita baseada em Shumway e Stoffer (1982) da seguinte forma:

i) Estimação do vetor de estados $\theta_{t}$ não observado:

Queremos estimar a série $\theta_{t}$ para $t=1,2, \ldots, n$ (suavização) e $t=n+1, n+$ $2, \ldots$ (previsão) utilizando a série observada $y_{t}$. Se os valores dos parâmetros $\varphi=\left(F_{t}, \beta_{j k}, V_{t}, W_{t}\right)$ são conhecidos, o estimador obtido pelo Filtro de Kalman pode ser calculado como a esperança condicional e será um estimador de mínimos quadrados. Na filosofia bayesiana, seria considerar o processo $\theta_{t}$ como um vetor de parâmetros aleatórios que depende de valores de parâmetros definidos a priori.

ii) Estimação dos parâmetros relacionados com as ondaletas, $\boldsymbol{\beta}_{j k}$, as matrizes de covariância dos erros, $V_{t}$ e $W_{t}$ e $F_{t}$ : 
Estimados através do algoritmo EM ("Esperança-Maximização").

iii) Estimação dos parâmetros relacionados com o vetor de estados no instante $t=0, \mu$ e $\Sigma$ :

Considerar a média $\mu$ do vetor $\theta_{0}$ como uma constante que será atualizada a cada iteração do Algoritmo EM pela média obtida através da suavização do Filtro de Kalman no tempo $t=1$. Considerar a matriz de covariâncias $\Sigma$ constante ao longo do processo. Em Shumway et al. (1981) encontra-se outro modo de estimar e testar hipóteses para a média e a covariância inicial do vetor de estado.

\section{Algoritmo EM}

Sejam as seguintes notações para as esperanças e covariâncias do vetor de espaços de estados, condicional ao vetor das observações:

i) $x_{t}^{s}=E\left(\theta_{t} \mid y_{1}, \ldots, y_{s}\right)$;

ii) $P_{t}^{s}=\operatorname{cov}\left(\theta_{t} \mid y_{1}, \ldots y_{s}\right)$;

iii) $P_{t, t-1}^{s}=\operatorname{cov}\left(\theta_{t}, \theta_{t-1} \mid y_{1}, \ldots, y_{s}\right)$.

Então podemos definir:

- $x_{t}^{t}$ e $P_{t}^{t}$ : estimadores obtidos pelo Filtro de Kalman usual:

- $x_{t}^{n}$ e $P_{t}^{n}, t \leq n$ : estimadores suavizados de erro quadrático médio mínimo relativos a $x_{t}$, média e matriz de covariâncias, respectivamente, baseados em todas as observações $y_{1}, \ldots, y_{n}$;

- $x_{t}^{n}$ e $P_{t}^{n}, t>n$ : previsão para o estado $\theta_{t}$ e respectiva matriz de covariância.

As equações do Filtro de Kalman estão no Capítulo 2.

Passos do Algoritmo EM:

Caso Geral. PASSO ZERO:

Calcular a função $\log$-verossimilhança conjunta completa $(\log L)$ de $\theta_{0}, \ldots, \theta_{n}$, $y_{1}, \ldots, y_{n} \operatorname{com}\left(\theta_{0} \mid D_{0}\right) \sim N(\mu, \Sigma)$ : 


$$
\begin{aligned}
\log L= & -(1 / 2) \log |\Sigma|-(1 / 2)\left(\theta_{0}-\mu\right)^{\prime} \Sigma^{-1}\left(\theta_{0}-\mu\right)-(n / 2) \log \left|W_{t}\right| \\
& -(1 / 2) \sum_{t=1}^{n}\left(\theta_{t}-G_{t} \theta_{t-1}\right)^{\prime} W_{t}^{-1}\left(\theta_{t}-G_{t} \theta_{t-1}\right)-(n / 2) \log \left|V_{t}\right| \\
& -(1 / 2) \sum_{t=1}^{n}\left(y_{t}-F_{t} \theta_{t}\right)^{\prime} V_{t}^{-1}\left(y_{t}-F_{t} \theta_{t}\right) .
\end{aligned}
$$

Como $\log L$ depende da série $\theta_{t}, t=1, \ldots, n$ não observada, aplicar o algoritmo EM condicional em relação a $y_{1}, \ldots, y_{n}$.

PASSO E:

Seja $H=E\left(\log L \mid y_{1}, \ldots, y_{n}\right)$. Então,

$$
\begin{aligned}
H= & -(1 / 2) \log |\Sigma|-(1 / 2) \operatorname{tr}\left[\Sigma^{-1}\left(P_{0}^{n}+\left(x_{0}^{n}-\mu\right)\left(x_{0}^{n}-\mu\right)^{\prime}\right]\right. \\
& -(n / 2) \log \left|W_{t}\right|-(1 / 2) \operatorname{tr}\left[\sum_{t=1}^{n} W_{t}^{-1}\left(C_{t}-B_{t} G_{t}^{\prime}-G_{t} B_{t}^{\prime}+G_{t} A_{t} G_{t}^{\prime}\right)\right] \\
& -(n / 2) \log \left|V_{t}\right|-(1 / 2) \operatorname{tr}\left[\sum_{t=1}^{n} V_{t}^{-1}\left(\left(y_{t}-F_{t} x_{t}^{n}\right)\left(y_{t}-F_{t} x_{t}^{n}\right)^{\prime}+F_{t} P_{t}^{n} F_{t}^{\prime}\right)\right]
\end{aligned}
$$

onde

$$
\begin{aligned}
& A_{t}=P_{t-1}^{n}+x_{t-1}^{n} x_{t-1}^{n^{\prime}}, \\
& B_{t}=P_{t, t-1}^{n}+x_{t}^{n} x_{t-1}^{n^{\prime}}, \\
& C_{t}=P_{t}^{n}+x_{t}^{n} x_{t}^{n^{\prime}} \mathrm{e}
\end{aligned}
$$

$x_{t}^{n}, P_{t}^{n}$ e $P_{t, t-1}^{n}$ são obtidos pelo suavizador de Kalman.

PASSO M:

Maximizar $H$ com relação a $F_{t}, G_{t}, V_{t}$ e $W_{t}$, que é equivalente a resolver as seguintes equações para $t=1, \ldots, n$ :

$$
\frac{\partial H}{\partial G_{t}}=0, \quad \frac{\partial H}{\partial F_{t}}=0, \quad \frac{\partial H}{\partial W_{t}}=0 \quad \text { e } \frac{\partial H}{\partial V_{t}}=0
$$


Apresentamos a seguir a solução das equações do passo M para uma particularização do modelo (4.2) supondo:

- uma única série temporal observada, isto é, $\mathrm{r}=1$;

- o vetor de estado univariado, isto é, $\mathrm{p}=1$;

$-F_{t}=F$;

$-V_{t}=V$;

$-W_{t}=W$

$-G_{t}=\sum_{j, k} \beta_{j k} \psi_{j, k}(t / T)$.

Podemos reescrever (4.4), a esperança condicional, como

$$
\begin{aligned}
H= & -(1 / 2) \log |\Sigma|-(1 / 2) \Sigma^{-1}\left(P_{0}^{n}+\left(x_{0}^{n}-\mu\right)^{2}\right)-(n / 2) \log |W| \\
& -(1 / 2) W^{-1} \sum_{t=1}^{n}\left[\left(C_{t}-2 B_{t} G_{t}+A_{t} G_{t}^{2}\right)\right]-(n / 2) \log |V| \\
& -(1 / 2) V^{-1} \sum_{t=1}^{n}\left[\left(y_{t}-F_{t} x_{t}^{n}\right)^{2}+P_{t}^{n} F_{t}^{2}\right] .
\end{aligned}
$$

Minimizando (4.5) com relação aos parâmetros $\beta_{j k}, F, V$ e $W$ obtemos:

i) Estimativas dos parâmetros $\beta_{j k}$ :

Substituímos os índices $\mathrm{j}, \mathrm{k}$ de $\beta_{j k}$ e $\psi_{j, k}(t / T)$ por um único índice $l$, onde $l=1, \ldots, s$ e $s$ é o número total de parâmetros $\beta_{j k}$. Deste modo teremos que resolver $s$ equações do tipo:

$$
\sum_{t=1}^{n}\left[B_{t} \psi_{l}(t / T)\right]-\sum_{t=1}^{n}\left[A_{t}\left(\sum_{i=1}^{s}\left(\hat{\beta}_{i} \psi_{i}(t / T)\right) \psi_{l}(t / T)\right]=0, \quad l=1, \ldots, s\right.
$$

Podemos montar um sistema para o conjunto das $s$ equações tal que $X \hat{\beta}=K$, onde

$$
X=\left(\begin{array}{cccc}
\sum_{t=1}^{n} A_{t} \psi_{1}^{2}(t / T) & \sum_{t=1}^{n} A_{t} \psi_{1}(t / T) \psi_{2}(t / T) & \ldots & \sum_{t=1}^{n} A_{t} \psi_{1}(t / T) \psi_{s}(t / T) \\
\sum_{t=1}^{n} A_{t} \psi_{1}(t / T) \psi_{2}(t / T) & \sum_{t=1}^{n} A_{t} \psi_{2}^{2}(t / T) & \ldots & \sum_{t=1}^{n} A_{t} \psi_{2}(t / T) \psi_{s}(t / T) \\
\vdots & \vdots & \ddots & \vdots \\
\sum_{t=1}^{n} A_{t} \psi_{1}(t / T) \psi_{s}(t / T) & \sum_{t=1}^{n} A_{t} \psi_{2}(t / T) \psi_{s}(t / T) & \ldots & \sum_{t=1}^{n} A_{t} \psi_{s}^{2}(t / T)
\end{array}\right.
$$




$$
\hat{\beta}=\left(\begin{array}{c}
\hat{\beta}_{1} \\
\hat{\beta}_{2} \\
\vdots \\
\hat{\beta}_{s}
\end{array}\right) \quad \text { e } \quad K=\left(\begin{array}{c}
\sum_{t=1}^{n} B_{t} \psi_{1}(t / T) \\
\sum_{t=1}^{n} B_{t} \psi_{2}(t / T) \\
\vdots \\
\sum_{t=1}^{n} B_{t} \psi_{s}(t / T)
\end{array}\right)
$$

E se existir a inversa de $\mathrm{X}$, temos $\hat{\beta}=X^{-1} K$.

ii) Estimativa do parâmetro $F$ :

$$
\hat{F}=\frac{\sum_{t=1}^{n} y_{t} x_{t}^{n}}{\sum_{t=1}^{n} C_{t}} .
$$

iii) Estimativa do parâmetro $W$ :

$$
\hat{W}=(1 / n) \sum_{t=1}^{n}\left[C_{t}-2 B_{t} \hat{G}_{t}+A_{t} \hat{G}_{t}^{2}\right]
$$

onde

$$
\hat{G}_{t}=\sum_{l=1}^{s} \hat{\beta}_{l} \psi_{l}(t / T) .
$$

iv) Estimativa do parâmetro $V$ :

$$
\hat{V}=(1 / n) \sum_{t=1}^{n}\left[\left(y_{t}-\hat{F}_{t} x_{t}^{n}\right)^{2}+P_{t}^{n} \hat{F}_{t}^{2}\right] .
$$




\subsection{Estudo de Simulação de Modelos AR(1) com Parâmetro Variando no Tempo}

Simulamos no programa S-Plus modelos autoregressivos de ordem $1 \mathrm{com}$ parâmetro variando no tempo. Consideramos que existe uma perturbação aleatória também na parte autoregressiva. Seja $y_{t}$ a série simulada. Utilizando a forma de espaço de estados, temos:

$$
\begin{aligned}
& y_{t}=F_{t} \theta_{t}+v_{t}, \\
& \theta_{t}=G_{t} \theta_{t-1}+w_{t}, \\
& v_{t} \sim N(0, V), \\
& w_{t} \sim N(0, W), \\
& F_{t}=1 \mathrm{e} \\
& G_{t} \text { é uma função do tempo. }
\end{aligned}
$$

Implementamos na linguagem Fortran 90 um programa que calcula as estimativas dos parâmetros utilizando o algoritmo EM e calcula o Filtro de Kalman no processo iterativo para estimar o estado. Estamos propondo que as funções que variam no tempo em $G_{t}$ sejam estimadas por uma soma de ondaletas, tal que

$$
\hat{G}_{t}=\sum_{j, k} \hat{\beta}_{j k} \psi_{j, k}(t / T)
$$

As ondaletas utilizadas foram:

- Haar:

$$
\psi_{j, k}(t)=\left\{\begin{array}{cl}
2^{j / 2}, & 2^{-j} k \leq t<2^{-j}(k+1 / 2) \\
-2^{j / 2}, & 2^{-j}(k+1 / 2) \leq t<2^{-j}(k+1) \\
0, & \text { caso contrário }
\end{array}\right.
$$

- Chapéu Mexicano:

$$
\psi_{j, k}(t)=2^{j / 2} \cdot\left(1-\left(2^{j} t-k\right)^{2}\right) \cdot \exp \left(-\left(2^{j} t-k\right)^{2} / 2\right)
$$


- Morlet ou Gaussiana Modulada:

$$
\psi_{j, k}(t)=2^{j / 2} \cdot \cos \left(w_{0}\left(2^{j} t-k\right)\right) \cdot \exp \left(-\left(2^{j} t-k\right)^{2} / 2\right)
$$

- Ondaleta Shannon:

$$
\psi_{j, k}(t)=\frac{2^{j / 2} \cdot \operatorname{sen}\left(\frac{\pi\left(2^{j} t-k\right)}{2}\right) \cdot \cos \left(\frac{3 \pi\left(2^{j} t-k\right)}{2}\right)}{\frac{\pi\left(2^{j} t-k\right)}{2}} .
$$

Realizamos várias simulações. Para cada uma das ondaletas utilizadas, consideramos variável a quantidade de parâmetros associados a elas. Seja a variação dos índices $j$ e $k$ das ondaletas da seguinte forma:

$$
\begin{aligned}
& j=1, \cdots, m, \\
& k=1, \cdots, 2^{j-1} .
\end{aligned}
$$

A variação do número de parâmetros foi feita de acordo com o total de dados $\left(T=256=2^{m}\right)$. Consideramos o valor máximo de $m$ a potência de dois do total de dados $T$. Ajustamos modelos para $m$ variando de 2 até 6 .

Foi implementado o procedimento de encolhimento de ondaletas ("wavelet shrinkage") com o objetivo de reduzir ou remover os ruídos presentes na estimação de $G_{t}$. Isto foi feito reduzindo (procedimento "soft") ou anulando (procedimento "hard") a magnitude dos coeficientes de ondaletas.

Usamos dois procedimentos:

- Encolhimento com limiar "hard": (mata ou preserva)

$$
\delta_{\lambda}^{H}(x)= \begin{cases}0, & -\lambda \leq x \leq \lambda \\ x, & x>\lambda \text { ou } x<-\lambda\end{cases}
$$

- Encolhimento com limiar "soft": (mata ou encolhe) 


$$
\delta_{\lambda}^{S}(x)= \begin{cases}0, & -\lambda \leq x \leq \lambda \\ x-\lambda, & x>\lambda \\ x+\lambda, & x<-\lambda\end{cases}
$$

onde $\lambda$ foi estimado por dois procedimentos:

- $\lambda$ universal (Donoho et al., 1995):

$$
\lambda=\sigma \cdot \sqrt{2 \log (T)}
$$

onde $T$ é o tamanho da série e $\sigma$ é o desvio padrão de todos os coeficientes das ondaletas.

- $\lambda$ variando de acordo com o nível $j$ :

$$
\lambda_{j}=\sigma_{j} \cdot \sqrt{2 \log \left(n_{j}\right)}
$$

onde $n_{j}$ é o total de coeficientes de ondaletas para o nível $j$ e $\sigma_{j}$ é o desvio padrão dos coeficientes das ondaletas para o nível $j$. Assim, combinando os tipos de limiar e os procedimentos de estimação de $\lambda$ temos quatro (4) tipos de encolhimento designados por "hard", "hardj", "soft" e "softj".

Os resultados obtidos são apresentados em uma tabela contendo o número de iterações até a convergência, o valor da verossimilhança $(-2 \log L)$ e as estimativas dos parâmetros $V$ e $W$ obtidas pelo algoritmo EM. Apresentamos o resultado do teste BIC para a escolha do melhor modelo. As estimativas do parâmetro $G_{t}$ e do estado do modelo são apresentadas graficamente, juntamente com o valor ajustado para $y_{t}$.

O critério de escolha do modelo é o BIC que aponta como melhor modelo aquele com menor valor da função

$$
B I C=-2 \log (\hat{L})+\operatorname{par} \cdot \log (T),
$$

onde $T$ é tamanho da série e par é o número total de parâmetros ( $V, W$ e os coeficientes das ondaletas). 
Tabela 4.1: Número de parâmetros para $m=2, \ldots, 6$.

\begin{tabular}{c|c|c|c}
\hline \hline $\mathrm{m}$ & $V, W$ & ondaletas & par \\
\hline \hline 2 & 2 & 3 & 5 \\
3 & 2 & 7 & 9 \\
4 & 2 & 15 & 17 \\
5 & 2 & 31 & 33 \\
6 & 2 & 63 & 65 \\
\hline \hline
\end{tabular}

A Tabela 4.1 acima apresenta o número de parâmetros para os diversos valores de $m$.

Foram analisadas três (3) séries simuladas com os seguintes valores para os parâmetros:

$$
\begin{aligned}
& F_{t}=1 ; \\
& V=0.16 \\
& W=0.25
\end{aligned}
$$

$\theta_{0}$ gerado pela distribuição Normal com média zero e variância 1 ;

$G_{t}$ séries dependentes do tempo:

Série 1:

$$
G_{t}=0.5(1-\cos (2 \pi t / T))
$$

Série 2:

$$
G_{t}=\cos (4 \pi t / T)
$$

Série 3:

$$
G_{t}= \begin{cases}2(t / T), & 0 \leq t / T<0.5 \\ -2(t / T)+2, & 0.5 \leq t / T \leq 1 \\ 0, & c c\end{cases}
$$

A seguir apresentamos a análise dos exemplos com os respectivos comentários. 
Série 1:

A Figura 4.1 apresenta os gráficos da série, estado e $G_{t}$. Utilizamos 20 modelos diferentes para obter estimativas dos parâmetros. Para cada uma das 4 ondaletas (Haar, Chapéu Mexicano, Morlet e Shannon) variamos o número de coeficientes das ondaletas $(m=2$ a $m=6)$.

A Tabela 4.2 apresenta os resultados obtidos para os modelos estimados. Observamos que as funções - $2 \log L$ diminuem a medida que introduzimos mais coeficientes para cada ondaleta, isto é, quando aumentamos o valor de $m$.

As estimativas de $V$ são razoáveis para todos os modelos e as estimativas de $W$ diminuem a medida que aumenta o número de coeficientes de ondaletas (para todas as ondaletas consideradas).

As estimativas de $G_{t}$ são apresentadas na Figura 4.2 para todos os modelos considerados. De um modo geral, as estimativas de $G_{t}$ para todas as estimativas e $m$ até 4 são razoáveis. Uma justificativa para as estimativas de $G_{t}$ serem ruins para os modelos com $m=5$ e $m=6$ é que a curva $G_{t}$ simulada é muito suave, necessitando de poucas ondaletas para estimá-la.

A Figura 4.3 apresenta as estimativas para $m=4$ para as 4 ondaletas consideradas e a utilização de limiares hard e soft. Os limiares hard e hardj funcionaram como suavisadores para as estimativas obtidas. Os limiares soft e softj alteraram bastante o valor das estimativas (subestimam no caso das ondaletas Haar, Morlet e Shannon e superestima no caso da ondaleta Chapéu Mexicano).

Segundo o critério BIC, o melhor modelo (comparando as 4 ondaletas) é o que utiliza a ondaleta Chapéu Mexicano com $m=2$. A Figura 4.4 apresenta as estimativas da série $y_{t}$, do estado $\theta_{t}$ e da matriz de transição $G_{t}$ para os melhores modelos para cada uma das 4 ondaletas consideradas. Observamos que em todos os casos obtivemos boas estimativas para a série e para o estado. Os modelos considerando as ondaletas Haar e Chapéu Mexicano estimam bem $G_{t}$ e os modelos Morlet e Shannon apresentam muito ruído na estimativa de $G_{t}$. 
Tabela 4.2: Resultados obtidos para a Série 1 utilizando os modelos com ondaletas Haar, Chapéu Mexicano, Morlet e Shannon, respectivamente $(m=2, \ldots, 6)$.

$\left({ }^{*}\right)$ Melhor modelo segundo o BIC.

\begin{tabular}{|c|c|c|c|c|c|}
\hline Modelo & Iteração & $-2 \log \mathrm{L}$ & W & V & $\mathrm{BIC}$ \\
\hline \multicolumn{6}{|c|}{ Ondaleta Haar } \\
\hline$\overline{m=2}$ & 101 & 98.7830 & 0.10558 & 0.31974 & 110.8242 \\
\hline $\mathrm{m}=3$ & 101 & 61.5202 & 0.11489 & 0.29177 & $83.1944\left(^{*}\right)$ \\
\hline $\mathrm{m}=4$ & 101 & 56.0345 & 0.08714 & 0.31172 & 96.9745 \\
\hline $\mathrm{m}=5$ & 101 & 36.0152 & 0.08558 & 0.28185 & 115.4872 \\
\hline $\mathrm{m}=6$ & 85 & -18.9929 & 0.02163 & 0.27510 & 137.5426 \\
\hline \multicolumn{6}{|c|}{ Ondaleta Chapéu Mexicano } \\
\hline $\mathrm{m}=2$ & 101 & 54.2750 & 0.16029 & 0.24338 & $66.3162\left(^{*}\right)$ \\
\hline $\mathrm{m}=3$ & 101 & 51.3250 & 0.18373 & 0.22142 & 72.9992 \\
\hline $\mathrm{m}=4$ & 101 & 52.0453 & 0.21232 & 0.19652 & 92.9854 \\
\hline $\mathrm{m}=5$ & 101 & 50.1955 & 0.21696 & 0.16296 & 129.6674 \\
\hline $\mathrm{m}=6$ & 54 & 16.2820 & 0.13579 & 0.21725 & 172.8176 \\
\hline \multicolumn{6}{|c|}{ Ondaleta Morlet } \\
\hline $\mathrm{m}=2$ & 101 & 96.8567 & 0.22722 & 0.24688 & 108.8979 \\
\hline $\mathrm{m}=3$ & 101 & 83.3653 & 0.13070 & 0.30931 & 105.0395 \\
\hline $\mathrm{m}=4$ & 101 & 76.3318 & 0.11033 & 0.31127 & 117.2719 \\
\hline $\mathrm{m}=5$ & 101 & 12.4175 & 0.01246 & 0.33637 & $91.8895\left(^{*}\right)$ \\
\hline $\mathrm{m}=6$ & 135 & -28.9097 & 0.00389 & 0.28825 & 127.6258 \\
\hline \multicolumn{6}{|c|}{ Ondaleta Shannon } \\
\hline$m=2$ & 101 & 91.4163 & 0.20508 & 0.25037 & 103.4576 \\
\hline$m=3$ & 101 & 80.6393 & 0.14482 & 0.28928 & 102.3135 \\
\hline $\mathrm{m}=4$ & 101 & 71.4335 & 0.08844 & 0.32749 & 112.3736 \\
\hline $\mathrm{m}=5$ & 101 & 9.8118 & 0.00890 & 0.33700 & $89.2837\left(^{*}\right)$ \\
\hline $\mathrm{m}=6$ & 214 & -43.7301 & 0.00074 & 0.26925 & 112.8055 \\
\hline
\end{tabular}


Serie 1
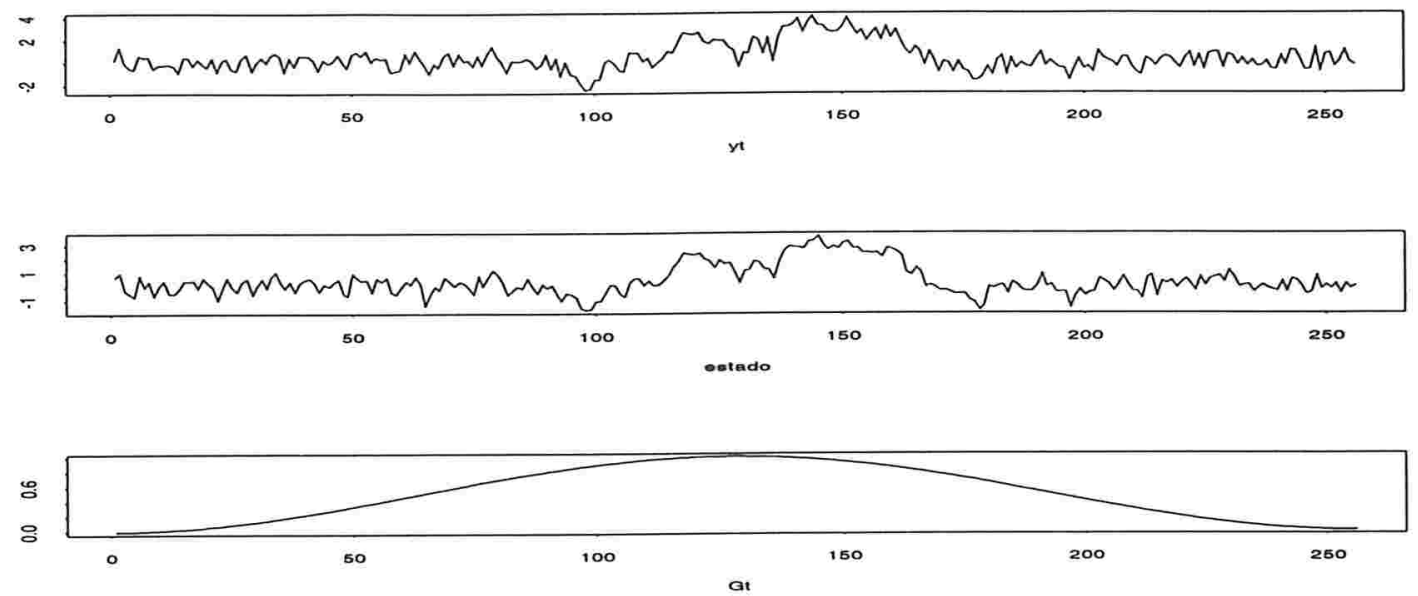

Figura 4.1: Gráficos da série $y_{t}$, estado $\theta_{t}$ e matriz de transição $G_{t}$ (Série 1).
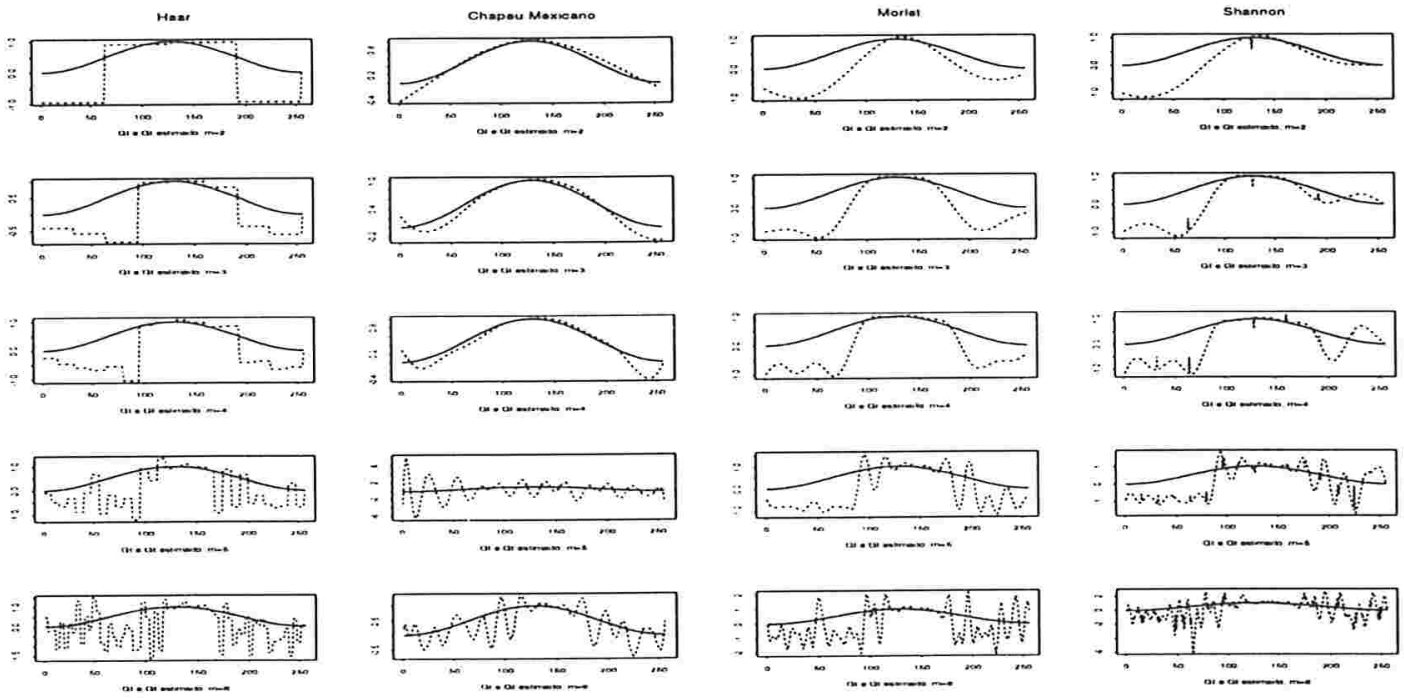

Figura 4.2: Gráficos das estimativas de $G_{t}$ para os diversos modelos ( $m=2$ até 6) utilizando as ondaletas Haar, Chapéu Mexicano, Morlet e Shannon (Série 1). 

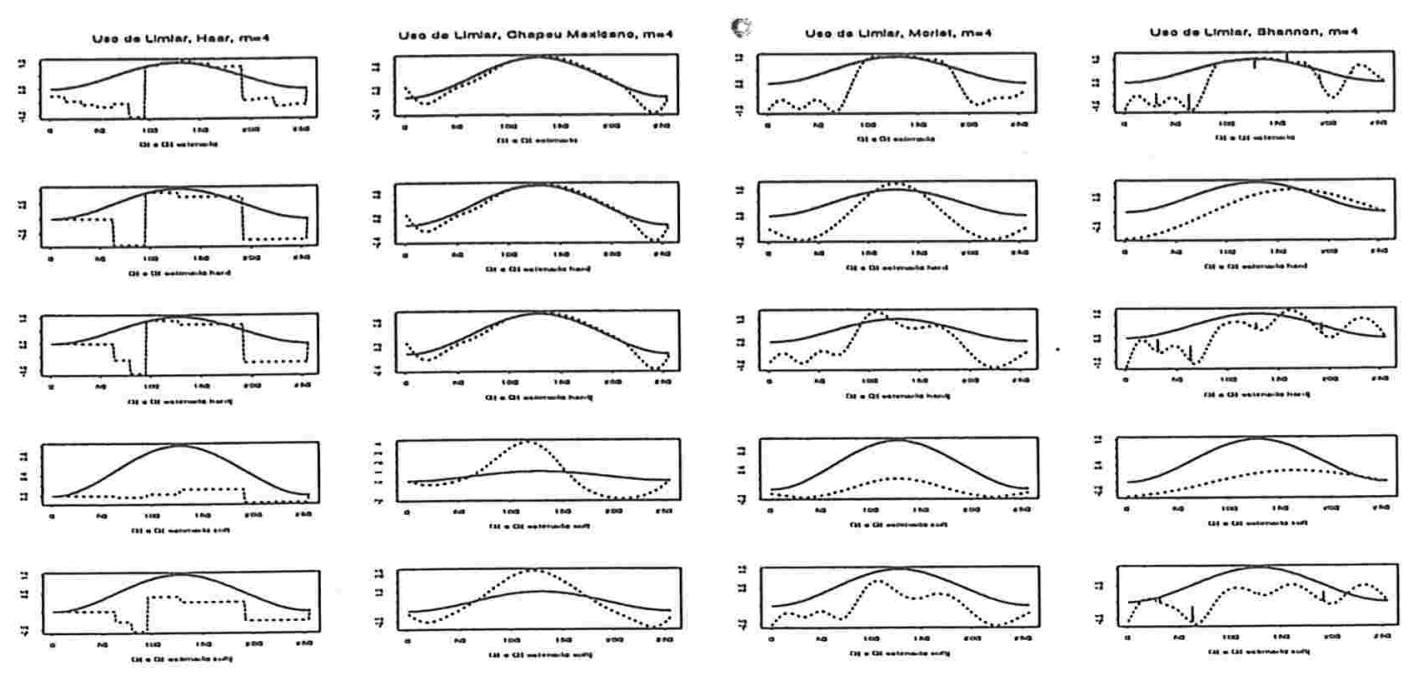

Figura 4.3: Gráficos das estimativas de $G_{t}$ utilizando limiares para os modelos com $m=4$ e as ondaletas Haar, Chapéu Mexicano, Morlet e Shannon (Série 1).
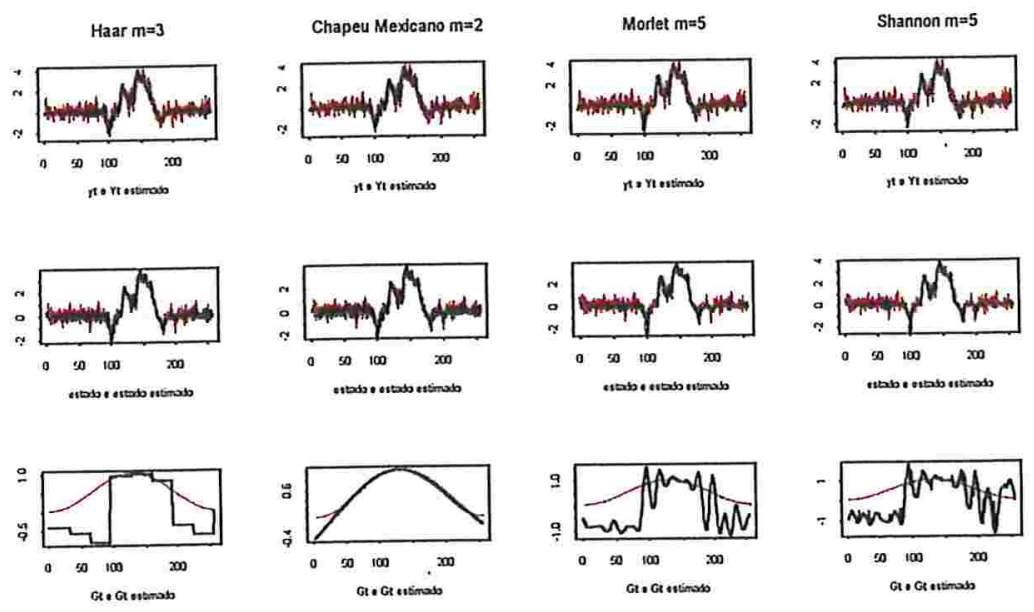

Figura 4.4: Gráficos da série $y_{t}$, estado $\theta_{t}$ e matriz de transição $G_{t}$ (vermelho) e suas respectivas estimativas (preto) para os modelos escolhidos como melhores segundo o critério BIC utilizando as ondaletas Haar, Chapéu Mexicano, Morlet e Shannon (Série 1). 
Série 2:

A Figura 4.5 apresenta a série, o estado e $G_{t}$. A Tabela 4.3 apresenta os resultados obtidos para todos os modelos utilizados. Observamos o mesmo comportamento da série anterior quanto aos valores da função $-2 \log L$ (diminuem para cada ondaleta com o aumento do número de coeficientes considerados).

As estimativas de $V$ e $W$ são razoáveis para todos os modelos (menos para o que utiliza a ondaleta Chapéu Mexicano com $m=2$ ).

As estimativas de $G_{t}$ são apresentadas na Figura 4.6 e a menos do modelo Chapéu Mexicano com $m=2$, todos os demais apresentam estimativas que acompanham o "desenho" da série $G_{t}$ original. Aqui também para $m=6$ encontramos um excesso de ruídos nas estimativas para todas as ondaletas.

A Figura 4.7 apresenta as estimativas de $G_{t}$ para $m=4$ para as 4 ondaletas e a utilização de limiares hard, hardj, soft e softj. Os limiares hard e soft tornaram zero os coeficientes estimados para as ondaletas Haar e Shannon. E de um modo geral pioraram o resultado obtido sem a utilização dos limiares.

A Figura 4.8 apresenta os melhores modelos (segundo o critério BIC) para as 4 ondaletas. Também aqui o melhor modelo foi o que considerou a ondaleta Chapéu Mexicano com $m=3$. Os ajustes foram muito bons, tanto para a série e o estado quanto para $G_{t}$. 
Tabela 4.3: Resultados obtidos para a Série 2 utilizando os modelos com ondaletas Haar, Chapéu Mexicano, Morlet e Shannon, respectivamente $(m=2, \ldots, 6)$.

$\left.{ }^{*}\right)$ Melhor modelo segundo o BIC.

\begin{tabular}{c|c|c|c|c|c}
\hline \hline Modelo & Iteração & $-2 \log L$ & $\mathrm{~W}$ & $\mathrm{~V}$ & $\mathrm{BIC}$ \\
& \multicolumn{5}{c}{ Ondaleta Haar } \\
\hline \hline $\mathrm{c}$ & & & & \\
\hline \hline $\mathrm{m}=2$ & 101 & 270.8763 & 0.10574 & 0.75370 & 282.9175 \\
$\mathrm{~m}=3$ & 101 & 108.7543 & 0.20884 & 0.24836 & 130.4285 \\
$\mathrm{~m}=4$ & 101 & 73.2336 & 0.30192 & 0.14003 & $114.1738\left(^{*}\right)$ \\
$\mathrm{m}=5$ & 101 & 51.8311 & 0.26460 & 0.13834 & 131.3031 \\
$\mathrm{~m}=6$ & 51 & 6.9101 & 0.15551 & 0.16453 & 163.4457 \\
\hline \hline \multicolumn{6}{c}{ Ondaleta Chapéu Mexicano } \\
\hline $\mathrm{m}=2$ & 157 & 305.0010 & 0.02377 & 1.12021 & 317.0422 \\
$\mathrm{~m}=3$ & 101 & 76.1537 & 0.27850 & 0.16325 & $97.8279\left(^{*}\right)$ \\
$\mathrm{m}=4$ & 102 & 69.3621 & 0.28121 & 0.15293 & 110.3023 \\
$\mathrm{~m}=5$ & 101 & 57.7630 & 0.27325 & 0.14257 & 137.2350 \\
$\mathrm{~m}=6$ & 52 & 42.3198 & 0.29817 & 0.10496 & 198.8554 \\
\hline \hline \multicolumn{6}{c}{ Ondaleta Morlet } \\
\hline \hline $\mathrm{m}=2$ & 101 & 173.2001 & 0.37963 & 0.25688 & 185.2413 \\
$\mathrm{~m}=3$ & 101 & 88.5536 & 0.35234 & 0.12688 & 110.2278 \\
$\mathrm{~m}=4$ & 101 & 66.0517 & 0.21607 & 0.19570 & $106.9919\left(^{*}\right)$ \\
$\mathrm{m}=5$ & 101 & 49.1009 & 0.14117 & 0.24189 & 128.5729 \\
$\mathrm{~m}=6$ & 210 & -39.2269 & 0.00143 & 0.27464 & 117.3087 \\
\hline \hline $\mathrm{m}=6$ & 51 & 32.7300 & 0.25050 & 0.11251 & 189.2656 \\
\hline \hline $\mathrm{m}=2$ & 101 & 156.7981 & 0.56186 & 0.08753 & 168.8394 \\
$\mathrm{~m}=3$ & 101 & 122.7814 & 0.51698 & 0.06152 & $144.4556\left(^{*}\right)$ \\
$\mathrm{m}=5$ & 101 & 112.5836 & 0.45943 & 0.08624 & 153.5237 \\
\hline \hline
\end{tabular}


Serie 2
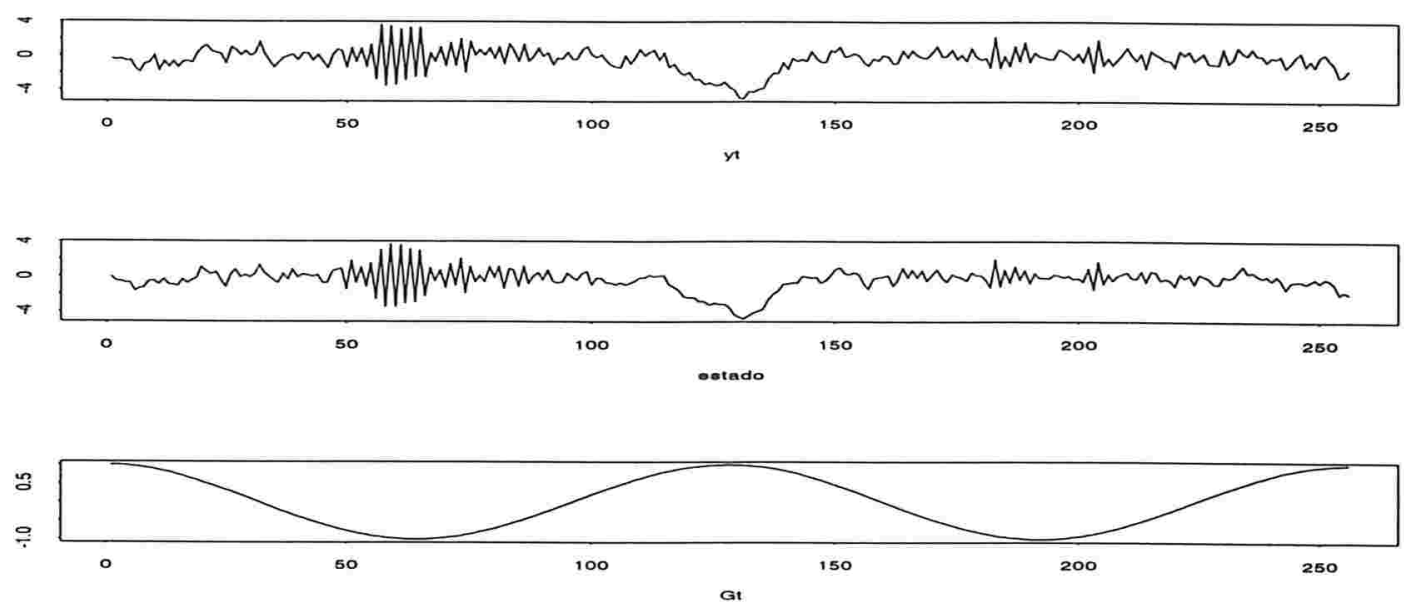

Figura 4.5: Gráficos da série $y_{t}$, estado $\theta_{t}$ e matriz de transição $G_{t}$ (Série 2).
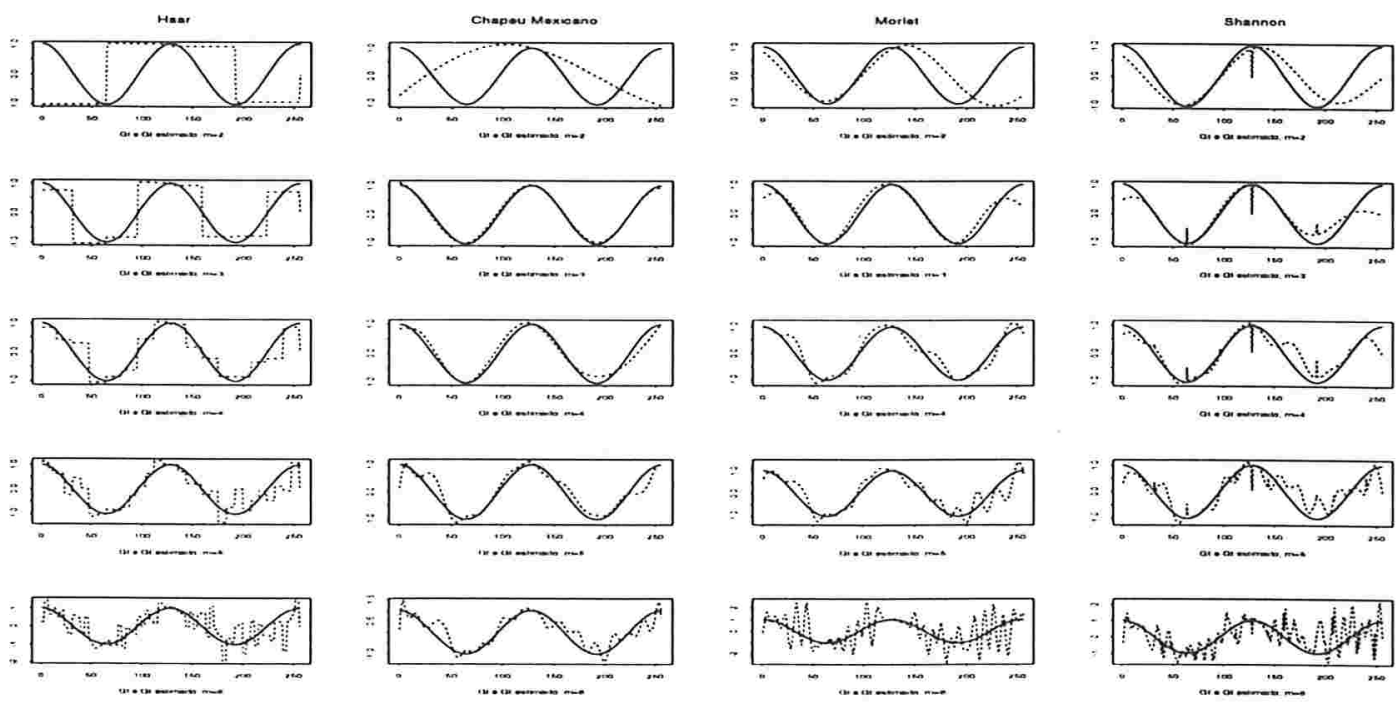

Figura 4.6: Gráficos das estimativas de $G_{t}$ para os diversos modelos ( $m=2$ até 6) utilizando as ondaletas Haar, Chapéu Mexicano, Morlet e Shannon (Série 2). 

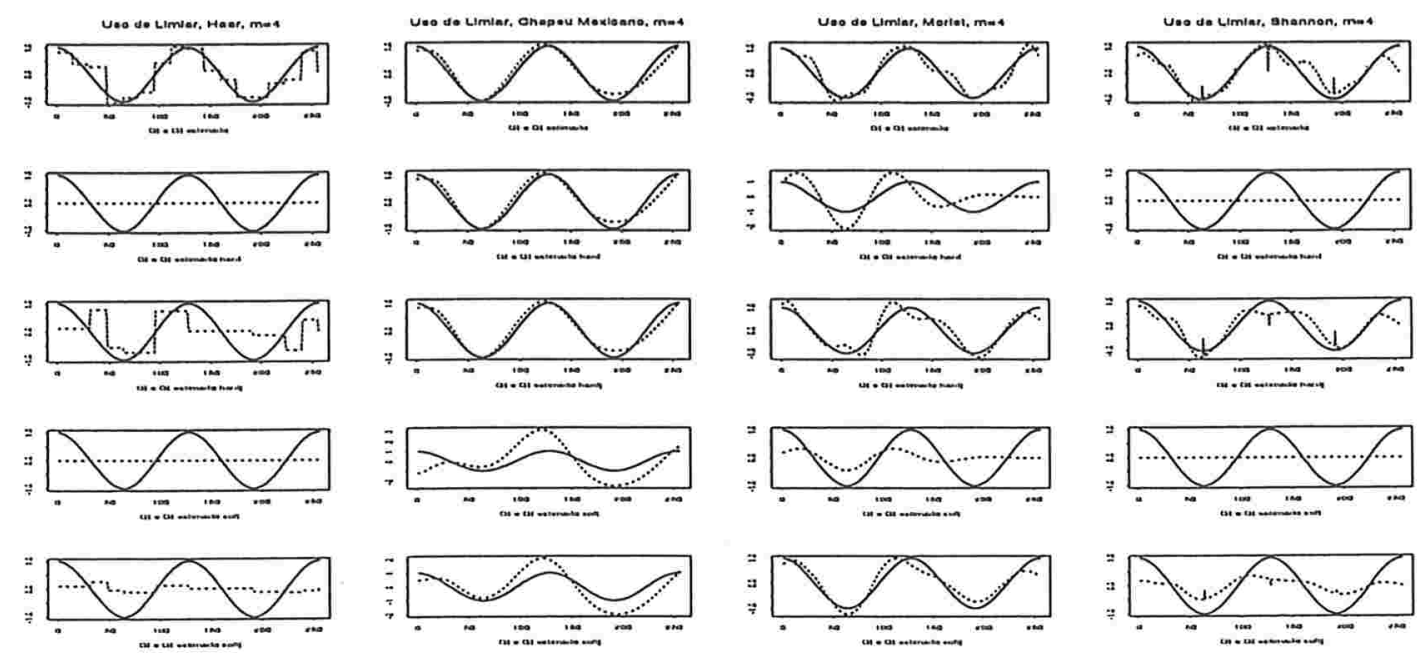

Figura 4.7: Gráficos das estimativas de $G_{t}$ utilizando limiares para os modelos com $m=4$ e as ondaletas Haar, Chapéu Mexicano, Morlet e Shannon (Série 2).

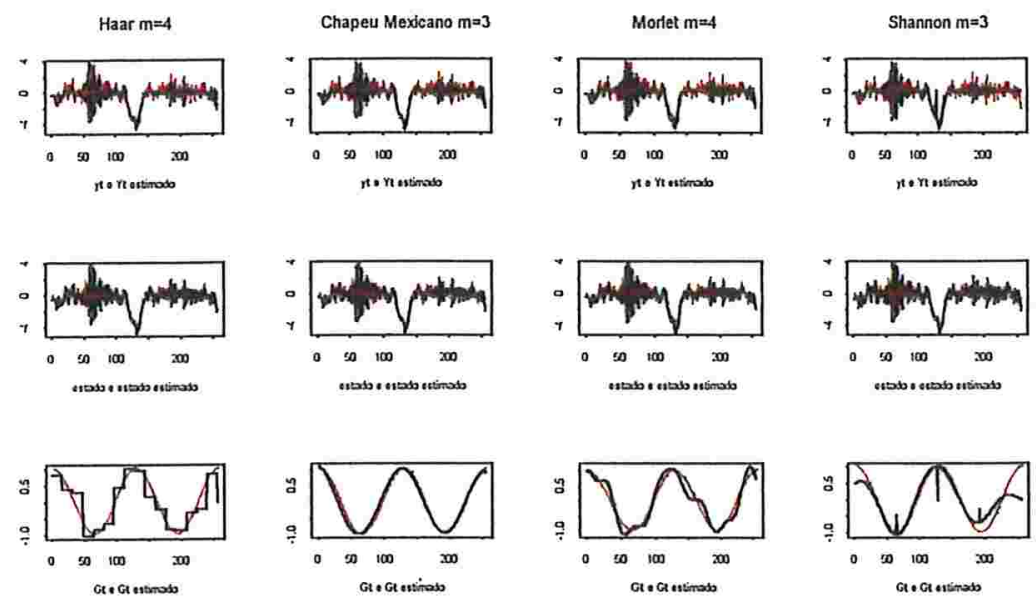

Figura 4.8: Gráficos da série $y_{t}$, estado $\theta_{t}$ e matriz de transição $G_{t}$ (vermelho) e suas respectivas estimativas (preto) para os modelos escolhidos como melhores segundo o critério BIC utilizando as ondaletas Haar, Chapéu Mexicano, Morlet e Shannon (Série 2). 
Série 3:

A Figura 4.9 apresenta a série 3 com seu respectivo estado e matriz de transição $G_{t}$.

Os resultados obtidos foram muito semelhantes aos da série 1, devido a estrutura adotada para $G_{t}$ que no caso anterior era mais suave.

Observamos da Tabela 4.4 que as estimativas de $W$ diminuem com o acréscimo de $m$ para as ondaletas Morlet e Shannon e este efeito não é observado para a variância $V$.

As Figura 4.10 e a Figura 4.11 apresentam o mesmo comportamento da Figura 4.2 e da Figura 4.3 respectivamente (relativas à série 1) para todas as ondaletas, menos para a Chapéu Mexicano.

Para esta série, o melhor modelo, segundo o critério BIC é o que utiliza a ondaleta Chapéu Mexicano com $m=2$. A Figura 4.12 traz as estimativas da série, do estado e de $G_{t}$ para os melhores modelos para cada tipo de ondaleta considerado.

\section{Comentários gerais:}

De um modo geral, as ondaletas consideradas na estimação conseguiram captar as mudanças da série $G_{t}$ ao longo do tempo. Como utilizamos séries suaves, não foi necessária a utilização de muitos coeficientes de ondaletas. O acréscimo de coeficientes acarretou uma diminuição da função $-2 \log L$ e do valor da estimativa da variância do estado $\theta_{t}, W$.

A utilização de limiares hard, hardj, soft e softj apresentou resultados muito diferentes dependendo do caso considerado, só melhorando a estimativa de $G_{t}$ quando a estimativa sem o limiar já era razoável (piorou nos outros casos).

O BIC como critério de escolha de melhor modelo pode estar sendo afetado pelo problema de diminuição da função $-2 \log L$ e da estimativa do parâmetro $W$ com o aumento da parametrização das ondaletas.

De um modo geral, obtemos boas estimativas das séries $y_{t}$ e dos estados $\theta_{t}$ (mesmo quando $G_{t}$ não era bem estimado). 
Tabela 4.4: Resultados obtidos para a Série 3 utilizando os modelos com ondaletas Haar, Chapéu Mexicano, Morlet e Shannon, respectivamente $(m=2, \ldots, 6)$.

$\left(^{*}\right)$ Melhor modelo segundo o BIC.

\begin{tabular}{|c|c|c|c|c|c|}
\hline Modelo & Iteração & $-2 \log L$ & W & V & $\mathrm{BIC}$ \\
\hline \multicolumn{6}{|c|}{ Ondaleta Haar } \\
\hline$m=2$ & 101 & 87.2046 & 0.07301 & 0.35151 & 99.2458 \\
\hline $\mathrm{m}=3$ & 101 & 57.6381 & 0.09955 & 0.30786 & $79.3123\left(^{*}\right)$ \\
\hline$m=4$ & 101 & 53.7639 & 0.07794 & 0.32396 & 94.9040 \\
\hline $\mathrm{m}=5$ & 101 & 33.2914 & 0.12849 & 0.24221 & 112.7634 \\
\hline $\mathrm{m}=6$ & 79 & -20.7488 & 0.03211 & 0.26073 & 135.7867 \\
\hline \multicolumn{6}{|c|}{ Ondaleta Chapéu Mexicano } \\
\hline $\mathrm{m}=2$ & 101 & 51.7052 & 0.15010 & 0.25352 & 63.7464(*) \\
\hline $\mathrm{m}=3$ & 101 & 49.7769 & 0.16790 & 0.23724 & 71.4511 \\
\hline $\mathrm{m}=4$ & 101 & 48.9981 & 0.18081 & 0.22404 & 89.9382 \\
\hline $\mathrm{m}=5$ & 101 & 47.3275 & 0.22683 & 0.18268 & 126.7995 \\
\hline $\mathrm{m}=6$ & 53 & 22.9681 & 0.21058 & 0.16025 & 179.5037 \\
\hline \multicolumn{6}{|c|}{ Ondaleta Morlet } \\
\hline$m=2$ & 101 & 82.4980 & 0.15342 & 0.30242 & $994.5392\left(^{*}\right)$ \\
\hline $\mathrm{m}=3$ & 101 & 75.9157 & 0.10487 & 0.33350 & 97.5899 \\
\hline $\mathrm{m}=4$ & 101 & 69.1832 & 0.07728 & 0.34370 & 110.1234 \\
\hline $\mathrm{m}=5$ & 101 & 14.1166 & 0.01525 & 0.33975 & 94.5885 \\
\hline $\mathrm{m}=6$ & 101 & -43.0084 & 0.00157 & 0.27358 & 113.5270 \\
\hline \multicolumn{6}{|c|}{ Ondaleta Shannon } \\
\hline$m=2$ & 101 & 80.9682 & 0.16510 & 0.28612 & 93.0094 \\
\hline$m=3$ & 101 & 73.5420 & 0.12627 & 0.30471 & 95.2161 \\
\hline$m=4$ & 101 & 65.6392 & 0.07218 & 0.34432 & 106.5793 \\
\hline$m=5$ & 101 & 11.1524 & 0.00831 & 0.39590 & $90.6243\left(^{*}\right)$ \\
\hline $\mathrm{m}=6$ & 208 & -55.4635 & 0.00070 & 0.26155 & 101.0720 \\
\hline
\end{tabular}



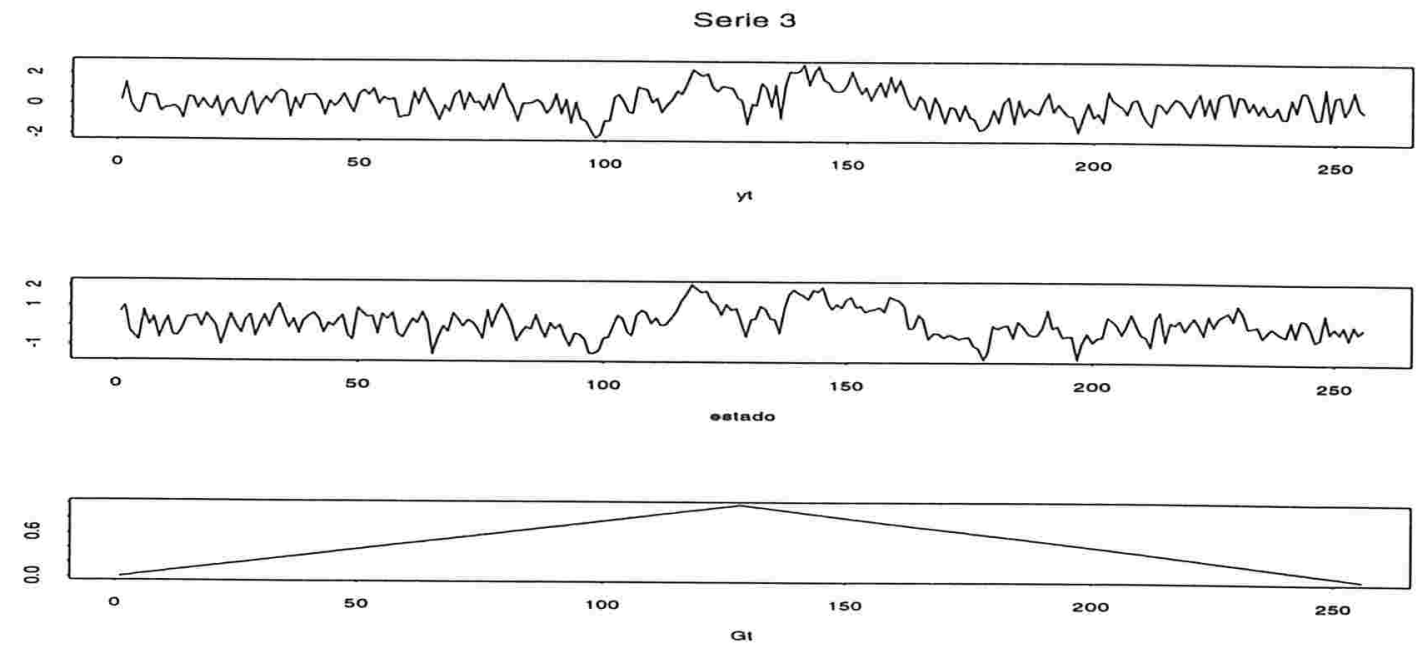

Figura 4.9: Gráficos da série $y_{t}$, estado $\theta_{t}$ e matriz de transição $G_{t}$ (Série 3).
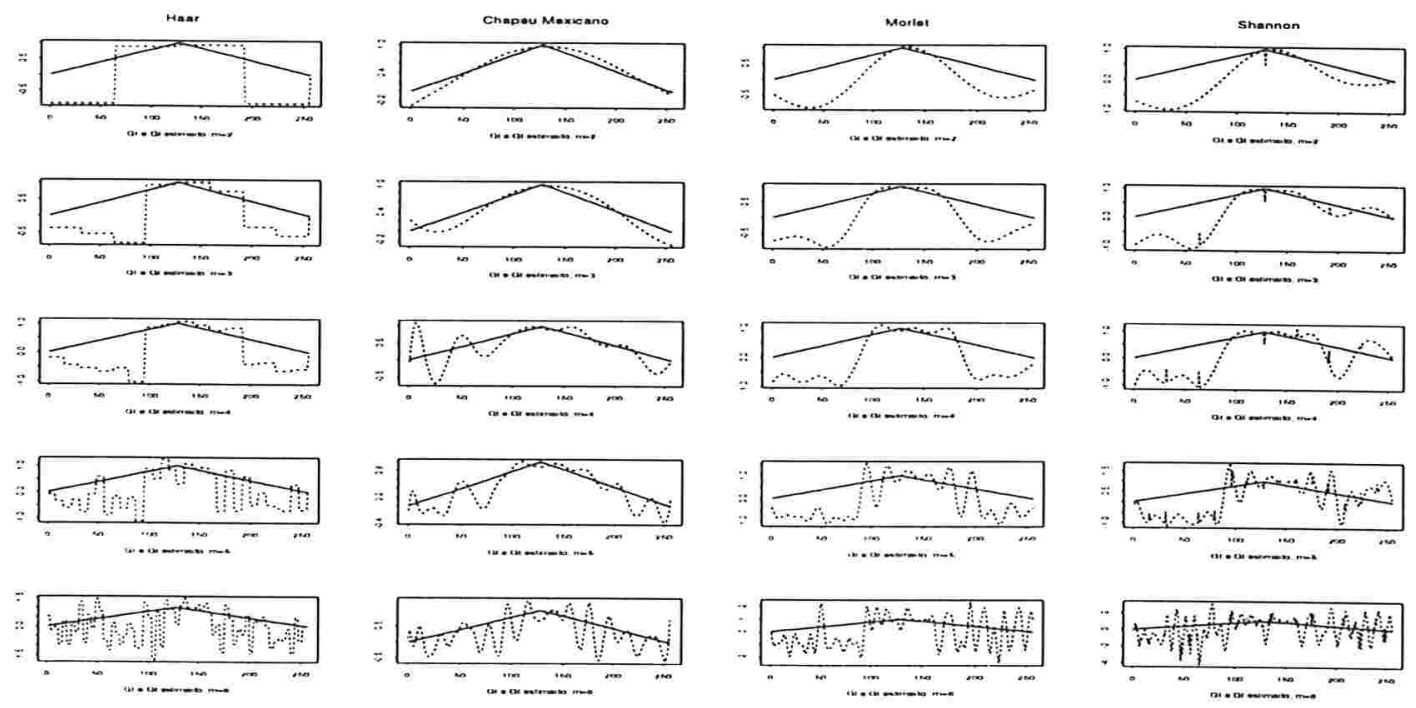

Figura 4.10: Gráficos das estimativas de $G_{t}$ para os diversos modelos ( $m=2$ até 6) utilizando as ondaletas Haar, Chapéu Mexicano, Morlet e Shannon (Série 3). 


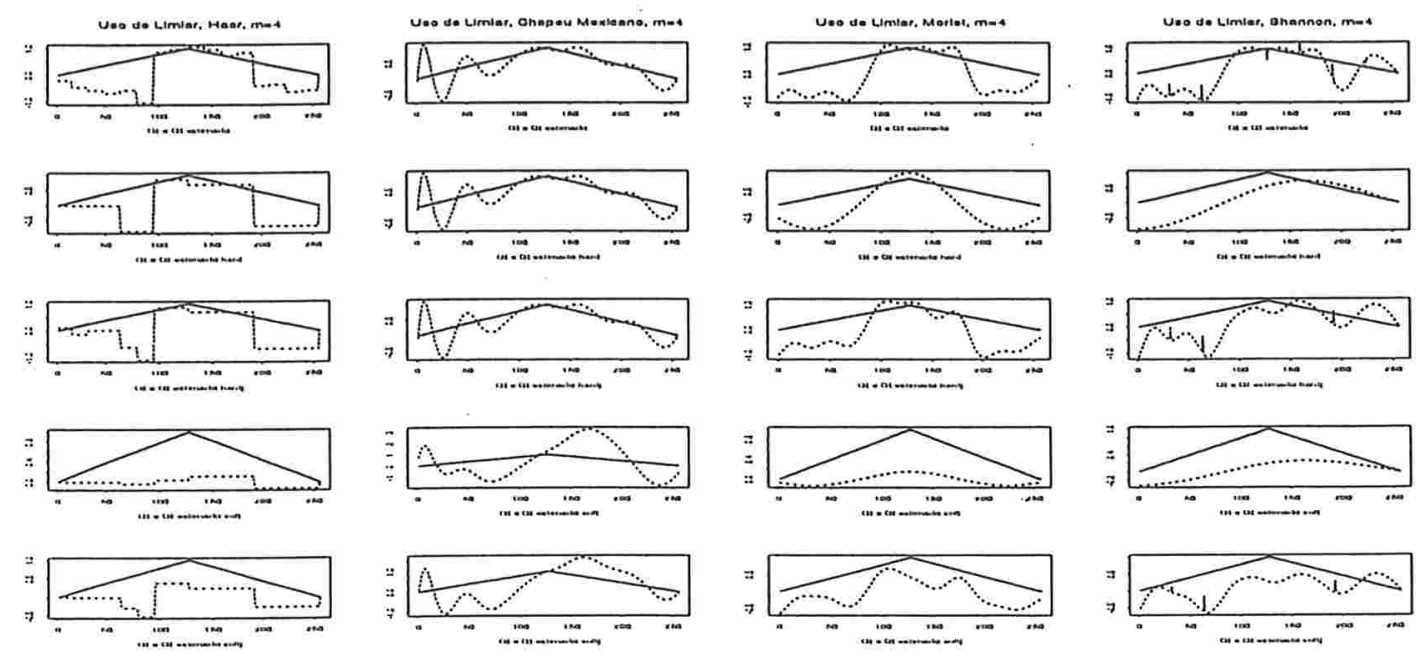

Figura 4.11: Gráficos das estimativas de $G_{t}$ utilizando limiares para os modelos com $m=4$ e as ondaletas Haar, Chapéu Mexicano, Morlet e Shannon (Série 3).

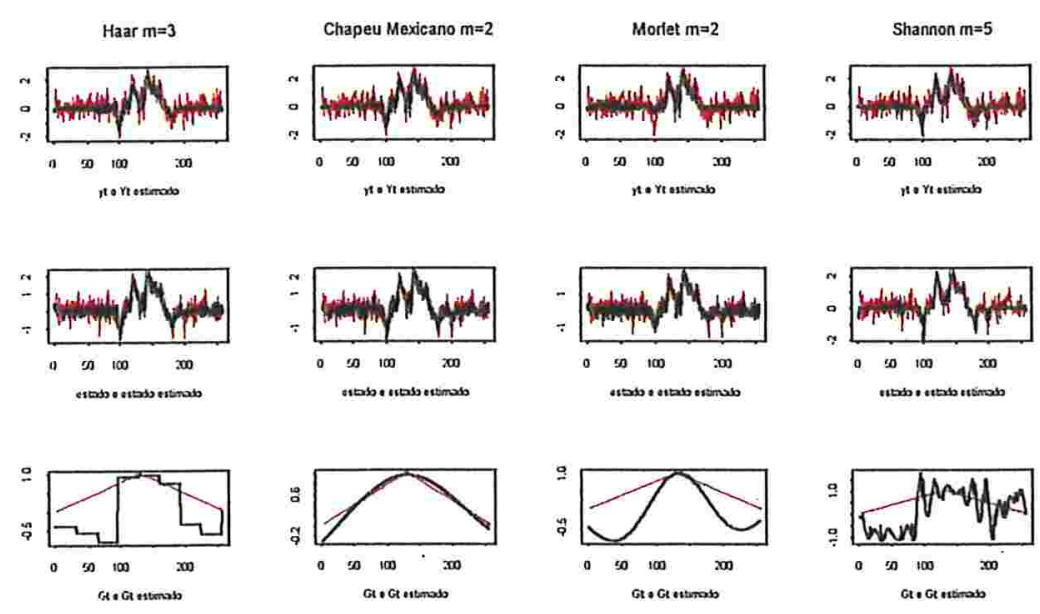

Figura 4.12: Gráficos da série $y_{t}$, estado $\theta_{t}$ e matriz de transição $G_{t}$ (vermelho) e suas respectivas estimativas (preto) para os modelos escolhidos como melhores segundo o critério BIC utilizando as ondaletas Haar, Chapéu Mexicano, Morlet e Shannon (Série 3). 


\section{Capítulo 5}

\section{Modelos com Mudança de Regime}

\subsection{Introdução}

Existem séries temporais que apresentam mudanças bruscas de comportamento ao longo do tempo. Muitos exemplos são encontrados em séries econométricas, onde guerras, mudanças de política econômica, política internacional, etc... podem causar essas mudanças.

Uma maneira de modelar mudanças de regime em séries temporais é assumir que existe variação de componentes do modelo em tempos indeterminados. $\mathrm{Na}$ maioria dos casos os pesquisadores têm pouca informação sobre os parâmetros causadores da mudança e existem testes estatísticos para detectar mudanças estruturais.

Pode-se abordar esse tipo de problema por diversas metodologias, tais como:

- associando teorias de cadeias de Markov e séries temporais;

- trabalhando com misturas de distribuição de probabilidade;

- utilizando a estrutura de modelos lineares dinâmicos na forma de espaço de estados.

Apresentamos a seguir algumas dessas abordagens.

O artigo de Shumway e Stoffer (1991) apresenta o problema de modelar mudanças de regime em séries temporais utilizando o modelo linear dinâmico na 
forma de espaço de estados. Neste modelo, a matriz do sistema $F_{t}$ varia no tempo de acordo com um processo aleatório independente.

O modelo é definido da seguinte forma:

$$
\begin{aligned}
& y_{t}=F_{t} \theta_{t}+v_{t} \mathrm{e} \\
& \theta_{t}=G_{t} \theta_{t-1}+w_{t},
\end{aligned}
$$

onde

i) $t=1, \ldots, n$;

ii) $v_{t} \sim$ iid $N\left(0, V_{t}\right)$;

iii) $w_{t} \sim \operatorname{iid} N\left(0, W_{t}\right)$;

iv) $v_{t}$ independente de $w_{t}$;

v) $\theta_{0} \sim N(\mu, \Sigma)$.

Para incorporar uma estrutura de variação ("switching") na matriz do sistema $F_{t}$, os autores assumiram que existem $M$ configurações possíveis que são estados de um processo não estacionário independente definido pelas probabilidades

$$
\pi_{J}(t)=P\left(F_{t}=M_{J}\right), \quad J=1, \ldots, M \text { e } t=1, \ldots, n,
$$

independentes das matrizes $F_{1}, \ldots, F_{t-1}$ e dos dados passados $y_{1}, \ldots, y_{t-1}$.

Uma informação importante sobre o estado atual é dado pelas probabilidades filtradas definidas para o estado $J$ como a probabilidade condicional

$$
\pi_{J}(t \mid t)=P\left(F_{t}=M_{J} \mid Y_{t}\right)
$$

onde $Y_{s}=\left\{y_{1}, y_{2}, \ldots, y_{s}\right\}, \quad s=1, \ldots, n$.

Shumway e Stoffer (1991) obtiveram estimadores (filtrados) para o vetor de estado e para as probabilidades associadas à forma da matriz $F_{t}$, usando o Filtro de Kalman e utilizaram um procedimento de estimação de máxima verossimilhança, o pseudo-algoritmo EM para a estimação dos parâmetros.

O artigo de Kim (1994) estende a idéia de Shumway e Stoffer (1991), introduzindo dependência no processo de mudança de regime e permitindo que a variação ocorra tanto na matriz do sistema $F_{t}$ quanto na matriz de transição $G_{t}$. O modelo usado foi 


$$
\begin{aligned}
& y_{t}=F_{S_{t}} \theta_{t}+\beta_{S_{t}} z_{t}+v_{t}, \\
& \theta_{t}=G_{S_{t}} \theta_{t-1}+\gamma_{S_{t}} z_{t}+w_{t}
\end{aligned}
$$

onde

i) $t=1, \ldots, n$;

ii) $v_{t} \sim$ iid $N\left(0, V_{t}\right)$;

iii) $w_{t} \sim$ iid $N\left(0, W_{t}\right)$;

iv) $v_{t}$ independente de $w_{t}$;

v) $\theta_{0} \sim N(\mu, \Sigma)$

vi) $z_{t}$ são variáveis exógenas defasadas ou não;

vii) $S_{t}$ é a variável aleatória que indica qual regime está em vigor no tempo $t$;

viii) $F_{S_{t}}, \beta_{S_{t}}, G_{S_{t}}$ e $\gamma_{S_{t}}$ são parâmetros que dependem de $S_{t}$.

Kim (1994) apresenta um algoritmo de filtragem e suavização para o modelo de espaço de estado com "switching" markoviano e obtém estimadores de máxima verossimilhança para os parâmetros desconhecidos. Ele utiliza o modelo para $S_{t}$ proposto por Hamilton (1989), que considera $S_{t}$ como resultado de um processo de Markov discreto de primeira ordem e $M$ estados cuja matriz de probabilidade de transição $\mathbb{P}$ é

$$
\mathbb{P}=\left(\begin{array}{cccc}
p_{11} & p_{12} & \ldots & p_{1 M} \\
p_{21} & p_{22} & \ldots & p_{2 M} \\
\vdots & \vdots & \ldots & \vdots \\
p_{M 1} & p_{M 2} & \ldots & p_{M M}
\end{array}\right)
$$

$\operatorname{com} p_{i j}=P\left(S_{t}=j \mid S_{t-1}=i\right)$ e $\sum_{j=1}^{M} p_{i j}=1, \quad \forall i$.

Os parâmetros $F_{S_{t}}, \beta_{S_{t}}, G_{S_{t}}$ e $\gamma_{S_{t}}$ são conhecidos para cada regime ou estado, mas o modelo é apropriado também para possíveis elementos desconhecidos.

O Capítulo 22 de Hamilton (1994) trata de modelos para séries temporais com mudanças de regime. Em Rabi (1998) encontramos um resumo das principais metodologias aplicadas a mudanças de regimes em séries temporais financeiras. 


\subsection{Modelagem com Ondaletas}

Seguindo a abordagem de Shumway e Stoffer (1991), propomos um modelo para as probabilidades $\pi_{J}(t)$ utilizando ondaletas. As ondaletas acrescentam uma variação ao longo do tempo nas probabilidades $\pi_{J}(t)$.

Para garantir que as estimativas pertençam ao intervalo $[0,1]$, apropriado para probabilidades, e a soma das probabilidades seja 1, utilizaremos uma reparametrização (ver página 134 de Agresti (1990)). A seguir apresentamos o modelo e os estimadores de máxima verossimilhança para os parâmetros. Utilizaremos o algoritmo EM e o Filtro de Kalman.

Considere o modelo

$$
\begin{aligned}
& y_{t}=F_{t} \theta_{t}+v_{t} \mathrm{e} \\
& \theta_{t}=G_{t} \theta_{t-1}+w_{t},
\end{aligned}
$$

sujeito às seguintes hipóteses:

i) $t=1, \ldots, n$;

ii) $v_{t} \sim$ iid $N\left(0, V_{t}\right)$;

iii) $w_{t} \sim$ iid $N\left(0, W_{t}\right)$;

iv) $v_{t}$ independente de $w_{t}$;

v) $\theta_{0} \sim N(\mu, \Sigma)$;

vi) $\pi_{J}(t)=P\left(F_{t}=M_{J}\right), \quad J=1, \ldots, M$ e $t=1, \ldots, n$;

vii) $\sum_{J=1}^{M} \pi_{J}(t)=1$;

viii) $\pi_{J}(t)=\frac{\exp \left(r_{J}(t)\right)}{\sum_{I=1}^{M} \exp \left(r_{I}(t)\right)}, \quad J=1, \ldots, M$;

ix) $r_{J}(t)=\sum_{l=1}^{s} \beta_{l}^{(J)} \psi_{l}(t / T), \quad J=1, \ldots, M-1$ e $r_{M}(t)=0$,

isto é,

$$
\begin{aligned}
\pi_{J}(t) & =\frac{\exp \left(\sum_{l=1}^{s} \beta_{l}^{(J)} \psi_{l}(t / T)\right)}{1+\sum_{I=1}^{M-1} \exp \left(\sum_{l=1}^{s} \beta_{l}{ }^{(I)} \psi_{l}(t / T)\right)}, \quad J=1, \ldots, M-1 \\
& =\frac{1}{1+\sum_{I=1}^{M-1} \exp \left(\sum_{l=1}^{s} \beta_{l}{ }^{(I)} \psi_{l}(t / T)\right)}, \quad J=M
\end{aligned}
$$


onde

$l=1, \ldots, s$ e $s$ é o número total de parâmetros $\beta_{j k}$;

$\beta_{j, k}$ são parâmetros associados às ondaletas;

$\psi_{j, k}(t / T)$ é a ondaleta no tempo $(t / T)$, fator de escala j e fator de translação $\mathrm{k}, \operatorname{com} T=n$.

Queremos estimar o vetor de estado $\theta_{t}$ e o vetor de parâmetros $\varphi=\left(\beta_{l}^{(J)}, G_{t}, V_{t}, W_{t}\right)$.

\subsection{Estimação dos Parâmetros}

A estimação dos parâmetros para o modelo apresentado em (5.1) será feita baseada em Shumway e Stoffer (1991) da seguinte forma:

i) Estimação do vetor de estados $\theta_{t}$ não observado:

Utilizaremos as equações do Filtro e Suavizador de Kalman que fornecem estimadores de mínimos quadrados. Estas equações consideram uma combinação ponderada dos $M$ possíveis valores da matriz $F_{t}$ e são uma extensão das equações clássicas do Filtro de Kalman. A dedução das equações encontra-se em Shumway e Stoffer (1991) e utiliza os estimadores para as probabilidades filtradas $\pi_{J}(t \mid t)$ dadas pelo modelo.

Considere a probabilidade condicional $\pi_{J}(t \mid t)$ dada pelo Teorema de Bayes (probabilidade a posteriori):

$$
\begin{gathered}
\pi_{J}(t \mid t)=P\left(F_{t}=M_{J} \mid Y_{t}\right), \quad \text { com } \quad Y_{t}=\left\{y_{1}, \ldots, y_{t}\right\} \\
\pi_{J}(t \mid t)=\frac{\pi_{J}(t) f_{J}(t \mid t-1)}{\sum_{i=1}^{M} \pi_{i}(t) f_{i}(t \mid t-1)},
\end{gathered}
$$

onde $f_{J}(t \mid t-1)$ é a densidade condicional de $y_{t}$ dado $F_{t}=M_{J}$ e o passado. Para o caso da hipótese de normal multivariada, supomos

$$
f_{J}(t \mid t-1) \sim N\left(M_{J} x_{t}^{t-1} ; \Sigma_{t J}=M_{J} P_{t}^{t-1} M_{J}^{\prime}+V_{t}\right),
$$

onde

i) $x_{t}^{t-1}=E\left(\theta_{t} \mid Y_{t-1}\right) \mathrm{e}$ 
ii) $P_{t}^{t-1}=\operatorname{cov}\left(\theta_{t} \mid Y_{t-1}\right)$, obtidos pelo filtro de Kalman.

Sejam as seguintes equações obtidas pelo filtro e suavizador de Kalman:

$$
\begin{gathered}
x_{t}^{t-1}=E\left(\theta_{t} \mid Y_{t-1}\right)=G_{t} x_{t-1}^{t-1}, \\
P_{t}^{t-1}=\operatorname{cov}\left(\theta_{t} \mid Y_{t-1}\right)=G_{t} P_{t-1}^{t-1} G_{t}^{\prime}+W_{t} \\
x_{t}^{t}=E\left(\theta_{t} \mid Y_{t}\right)=x_{t}^{t-1}+\sum_{J=1}^{M} \pi_{J}(t \mid t) K_{t J}\left(y_{t}-M_{J} x_{t}^{t-1}\right) \mathrm{e} \\
P_{t}^{t}=\operatorname{cov}\left(\theta_{t} \mid Y_{t}\right)=\sum_{J=1}^{M} \pi_{J}(t \mid t)\left(I-K_{t J} M_{J}\right) P_{t}^{t-1},
\end{gathered}
$$

onde

$$
\begin{aligned}
& Y_{t}=\left\{y_{1}, y_{2}, \ldots, y_{t}\right\}, \\
& K_{t J}=P_{t}^{t-1} M_{J}^{\prime}\left(M_{J} P_{t}^{t-1} M_{J}^{\prime}+V_{t}\right)^{-1} \text { é o ganho do filtro, } \\
& x_{0}^{0}=\mu \mathrm{e} \\
& P_{0}^{0}=\Sigma .
\end{aligned}
$$

Equações de Suavização: para $t=n, n-1, \ldots$, , (1)

$$
\begin{gathered}
x_{t-1}^{n}=x_{t-1}^{t-1}+J_{t-1}\left(x_{t}^{n}-x_{t}^{t-1}\right) \mathrm{e} \\
P_{t-1}^{n}=P_{t-1}^{t-1}+J_{t-1}\left(P_{t}^{n}-P_{t}^{t-1}\right) J_{t-1}^{\prime},
\end{gathered}
$$

onde

$$
\begin{aligned}
& J_{t-1}=P_{t-1}^{t-1} G_{t}^{\prime}\left(P_{t}^{t-1}\right)^{-1} \\
& P_{t-1, t-2}^{n}=P_{t-1}^{t-1} J_{t-2}^{\prime}+J_{t-1}\left(P_{t, t-1}^{n}-G_{t} P_{t-1}^{t-1}\right) J_{t-2}^{\prime} \mathrm{e} \\
& P_{n, n-1}^{n}=\sum_{J=1}^{M} \pi_{J}(n \mid n)\left(I-K_{n J} M_{J}\right) G_{t} P_{n-1}^{n-1}, \\
& \mathrm{I}=\text { matriz identidade. }
\end{aligned}
$$


ii) Estimação dos parâmetros relacionados com as ondaletas, $\beta_{l}^{(J)}$, as matrizes de covariância dos erros, $V_{t}$ e $W_{t}$ e a matriz de transição $G_{t}$ :

Utilizaremos o algoritmo EM.

Caso Geral. PASSO ZERO:

Calcular a função $\log$-verossimilhança conjunta completa $(\log L)$ de $\theta_{0}, \ldots, \theta_{n}$, $y_{1}, \ldots, y_{n} \operatorname{com}\left(\theta_{0} \mid D_{0}\right) \sim N(\mu, \Sigma)$ e $D_{0}$ é o conjunto das informações no tempo $t=0$.

$$
\begin{aligned}
\log L= & -(1 / 2) \log |\Sigma|-(1 / 2)\left(\theta_{0}-\mu\right)^{\prime} \Sigma^{-1}\left(\theta_{0}-\mu\right)-(n / 2) \log \left|W_{t}\right| \\
& -(1 / 2) \sum_{t=1}^{n}\left(\theta_{t}-G_{t} \theta_{t-1}\right)^{\prime} W_{t}^{-1}\left(\theta_{t}-G_{t} \theta_{t-1}\right) \\
& +\sum_{t=1}^{n} \sum_{J=1}^{M} I\left(F_{t}=M_{J}\right) \log \left(\pi_{J}(t)\right)-(n / 2) \log \left|V_{t}\right| \\
& -(1 / 2) \sum_{t=1}^{n} \sum_{J=1}^{M} I\left(F_{t}=M_{J}\right)\left(y_{t}-F_{t} \theta_{t}\right)^{\prime} V_{t}^{-1}\left(y_{t}-F_{t} \theta_{t}\right)
\end{aligned}
$$

onde $I\left(F_{t}=M_{J}\right)$ é uma função indicadora.

Como $\log L$ depende da série $\theta_{t}, t=1, \ldots, n$ não observada, aplicamos o algoritmo EM condicional com relação a $y_{1}, \ldots, y_{n}$.

PASSO E:

Sejam $H=E\left(\log L \mid y_{1}, \ldots, y_{n}\right)$, isto é, a esperança condicional da função $\log L$ e $E\left(I\left(F_{t}=M_{J}\right) \mid Y_{n}\right)=\pi_{J}(t \mid n)$.

Então:

$$
\begin{aligned}
H= & -(1 / 2) \log |\Sigma|-(1 / 2) \operatorname{tr}\left[\Sigma^{-1}\left(P_{0}^{n}+\left(x_{0}^{n}-\mu\right)\left(x_{0}^{n}-\mu\right)^{\prime} \mid\right]\right. \\
& -(n / 2) \log \left|W_{t}\right|-(1 / 2) \operatorname{tr}\left[W_{t}^{-1}\left(C-B G_{t}^{\prime}-G_{t} B^{\prime}+G_{t} A G_{t}^{\prime}\right)\right] \\
& +\sum_{t=1}^{n} \sum_{J=1}^{M} \pi_{J}(t \mid n) \log \left(\pi_{J}(t)\right)-(n / 2) \log \left|V_{t}\right| \\
& -(1 / 2) \operatorname{tr}\left[\sum_{t=1}^{n} \sum_{J=1}^{M} V_{t}^{-1} \pi_{J}(t \mid n)\left(\left(y_{t}-M_{J} x_{t}^{n}\right)\left(y_{t}-M_{J} x_{t}^{n}\right)^{\prime}+M_{J} P_{t}^{n} M_{J}^{\prime}\right)\right]
\end{aligned}
$$


onde

$$
\begin{aligned}
& A=\sum_{t=1}^{n}\left(P_{t-1}^{n}+x_{t-1}^{n} x_{t-1}^{n^{\prime}}\right), \\
& B=\sum_{t=1}^{n}\left(P_{t, t-1}^{n}+x_{t}^{n} x_{t-1}^{n^{\prime}}\right), \\
& C=\sum_{t=1}^{n}\left(P_{t}^{n}+x_{t}^{n} x_{t}^{n^{\prime}}\right) \mathrm{e}
\end{aligned}
$$

$x_{t}^{n}, P_{t}^{n}$ e $P_{t, t-1}^{n}$ são obtidos pelo suavizador de Kalman.

Shumway e Stoffer (1991) utilizam o que eles chamaram de "pseudo-EM" devido à substituição de $\pi_{J}(t \mid n)$ por $\pi_{J}(t \mid t)$. A recursão para trás das probabilidades filtradas envolve integração de misturas de normais e pode ser complicado obtêla. Os autores ainda sugerem a utilização de técnicas de integração via Monte Carlo como o amostrador de Gibbs, embora utilizem a aproximação mencionada. $\pi_{J}(t \mid t)$ será obtida pela probabilidade condicional (usar $\pi_{J}(t)$ no passo anterior).

PASSO M:

Maximizar $H$ com relação a $G_{t}, V_{t}, W_{t}$ e $\beta_{r}^{(J)}$ que é equivalente a resolver as seguintes equações para $t=1, \ldots, n$ :

$$
\frac{\partial H}{\partial G_{t}}=0, \quad \frac{\partial H}{\partial W_{t}}=0, \quad \frac{\partial H}{\partial V_{t}}=0 \quad \text { e } \frac{\partial H}{\partial \beta_{r}^{(J)}}=0
$$

i) Estimativa do parâmetro $G_{t}$ :

$$
\hat{G}_{t}=B A^{-1}
$$

ii) Estimativa do parâmetro $W_{t}$ :

$$
\hat{W}_{t}=(1 / n)\left(C-B \hat{G}_{t}{ }^{\prime}-\hat{G}_{t} B^{\prime}+\hat{G}_{t} A \hat{G}_{t}{ }^{\prime}\right)
$$

iii) Estimativa do parâmetro $V_{t}$ : 


$$
\hat{V}_{t}=(1 / n) \sum_{t=1}^{n} \sum_{J=1}^{M} \pi_{J}(t \mid t)\left[\left(y_{t}-M_{J} x_{t}^{n}\right)\left(y_{t}-M_{J} x_{t}^{n}\right)^{\prime}+M_{J} P_{t}^{n} M_{J}^{\prime}\right]
$$

vi) Estimativa dos parâmetros $\beta_{r}^{(J)}$ :

Maximizar $H$ em relação a $\pi_{J}(t)$; considerar

$$
\pi_{J}(t)=\frac{\exp \left(\sum_{l=1}^{s} \beta_{l}^{(J)} \psi_{l}(t / T)\right)}{1+\sum_{I=1}^{M-1} \exp \left(\sum_{l=1}^{s} \beta_{l}{ }^{(I)} \psi_{l}(t / T)\right)}, \quad J=1, \ldots, M-1
$$

e

$$
\pi_{M}(t)=\frac{1}{1+\sum_{I=1}^{M-1} \exp \left(\sum_{l=1}^{s} \beta_{l}^{(I)} \psi_{l}(t / T)\right)} .
$$

Então,

$$
\log \left(\pi_{J}(t)\right)=\left(\sum_{l=1}^{s} \beta_{l}^{(J)} \psi_{l}(t / T)\right)-\log \left(1+\sum_{I=1}^{M-1} \exp \left(\sum_{l=1}^{s} \beta_{l}{ }^{(I)} \psi_{l}(t / T)\right)\right), \quad J=1, \ldots, M-1
$$

$\mathrm{e}$

$$
\log \left(\pi_{M}(t)\right)=-\log \left(1+\sum_{I=1}^{M-1} \exp \left(\sum_{l=1}^{s} \beta_{l}{ }^{(I)} \psi_{l}(t / T)\right)\right)
$$

A derivada parcial de $H$ em relação a $\beta_{r}^{(J)}$, para $J=1, \ldots, M-1$ e $r=1, \ldots, s$, é dada por:

$$
\frac{\partial H}{\partial \beta_{r}^{(J)}}=\sum_{t=1}^{n}\left[\frac{\partial}{\partial \beta_{r}^{(J)}} \sum_{I=1}^{M} \pi_{I}(t \mid t) \log \left(\pi_{I}(t)\right)\right]=0,
$$

onde

$$
\begin{gathered}
\sum_{t=1}^{n} \sum_{I=1}^{M} \pi_{I}(t \mid t) \log \left(\pi_{I}(t)\right)=\sum_{t=1}^{n}\left[\sum_{I=1}^{M-1} \pi_{I}(t \mid t) \sum_{l=1}^{s}\left(\beta_{l}{ }^{(I)} \psi_{l}(t / T)\right)-\right. \\
\log \left(1+\sum_{I=1}^{M-1} \exp \left(\sum_{l=1}^{s} \beta_{l}{ }^{(I)} \psi_{l}(t / T)\right) \cdot \sum_{I=1}^{M} \pi_{I}(t \mid t)\right]
\end{gathered}
$$




$$
\begin{gathered}
\frac{\partial}{\partial \beta_{r}^{(J)}} \sum_{I=1}^{M} \pi_{I}(t \mid t) \log \left(\pi_{I}(t)\right)=\pi_{J}(t \mid t) \psi_{r}(t / T)-\left(1+\sum_{I=1}^{M-1} \exp \left(\sum_{l=1}^{s} \beta_{l}^{(I)} \psi_{l}(t / T)\right)\right)^{-1} \times \\
\exp \left(\sum_{l=1}^{s} \beta_{l}^{(J)} \psi_{l}(t / T)\right) \psi_{r}(t / T) .
\end{gathered}
$$

Temos que resolver um sistema não linear com $(M-1) . s$ equações dadas abaixo:

$$
\sum_{t=1}^{n}\left[\frac{\exp \left(\sum_{l=1}^{s} \beta_{l}^{(J)} \psi_{l}(t / T)\right) \psi_{r}(t / T)}{\left(1+\sum_{I=1}^{M-1} \exp \left(\sum_{l=1}^{s} \beta_{l}^{(I)} \psi_{l}(t / T)\right)\right.}-\pi_{J}(t \mid t) \psi_{r}(t / T)\right]=0
$$

Sejam

$$
\hat{\beta}^{(J)}=\left(\begin{array}{c}
\hat{\beta}_{1}^{(J)} \\
\hat{\beta}_{2}^{(J)} \\
\vdots \\
\hat{\beta}_{s}^{(J)}
\end{array}\right) \quad \text { e } \quad X_{t}=\left(\begin{array}{c}
\psi_{1}(t / T) \\
\psi_{2}(t / T) \\
\vdots \\
\psi_{s}(t / T)
\end{array}\right)
$$

Desta forma, o sistema passa a ser

$$
\begin{gathered}
\sum_{t=1}^{n}\left[\frac{\left(\exp \left(X_{t}^{\prime} \hat{\beta}^{(J)}\right) \psi_{r}(t / T)\right.}{\left(1+\sum_{I=1}^{M-1} \exp \left(X_{t}^{\prime} \hat{\beta}^{(I)}\right)\right.}-\pi_{J}(t \mid t) \psi_{r}(t / T)\right]=0 . \\
J=1, \ldots, M-1 \text { e } r=1, \ldots, s .
\end{gathered}
$$

Para resolvermos esse sistema devemos recorrer a algum procedimento numérico.

CASO PARTICULAR: $M=2, J=1$.

$$
\pi_{1}(t)=\pi(t)=\frac{\exp \left(\sum_{l=1}^{s} \beta_{l} \psi_{l}(t / T)\right)}{1+\exp \left(\sum_{l=1}^{s} \beta_{l} \psi_{l}(t / T)\right)}
$$




$$
\begin{gathered}
\pi_{2}(t)=1-\pi_{1}(t) \\
=1-\frac{\exp \left(\sum_{l=1}^{s} \beta_{l} \psi_{l}(t / T)\right)}{1+\exp \left(\sum_{l=1}^{s} \beta_{l} \psi_{l}(t / T)\right)} \\
=\frac{1}{1+\exp \left(\sum_{l=1}^{s} \beta_{l} \psi_{l}(t / T)\right)}, \\
\pi_{1}(t \mid t)=\pi(t \mid t)=\frac{\pi(t) f_{1}(t \mid t-1)}{\pi(t)\left(f_{1}(t \mid t-1)-f_{2}(t \mid t-1)\right)+f_{2}(t \mid t-1)} \mathrm{e} \\
\pi_{2}(t \mid t)=\frac{(1-\pi(t)) f_{2}(t \mid t-1)}{\pi(t)\left(f_{1}(t \mid t-1)-f_{2}(t \mid t-1)\right)+f_{2}(t \mid t-1)} .
\end{gathered}
$$

Temos que resolver um sistema não linear com $s$ equações, dadas abaixo:

$r=1, \ldots, s$.

$$
\sum_{t=1}^{n}\left[\frac{\exp \left(\sum_{l=1}^{s} \beta_{l} \psi_{l}(t / T)\right) \psi_{r}(t / T)}{\left(1+\exp \left(\sum_{l=1}^{s} \beta_{l} \psi_{l}(t / T)\right)\right.}-\pi(t \mid t) \psi_{r}(t / T)\right]=0,
$$

Considerando

temos

$$
\hat{\beta}=\left(\begin{array}{c}
\hat{\beta}_{1} \\
\hat{\beta}_{2} \\
\vdots \\
\hat{\beta}_{s}
\end{array}\right) \quad \text { e } \quad X_{t}=\left(\begin{array}{c}
\psi_{1}(t / T) \\
\psi_{2}(t / T) \\
\vdots \\
\psi_{s}(t / T)
\end{array}\right)
$$

$$
\begin{aligned}
& \sum_{t=1}^{n}\left(\frac{\exp \left(X_{t}^{\prime} \hat{\beta}\right) \psi_{1}(t / T)}{1+\exp \left(X_{t}^{\prime} \hat{\beta}\right)}\right) \sum_{t=1}^{n} \pi(t \mid t) \psi_{1}(t / T) \\
& \vdots \vdots \\
& \sum_{t=1}^{n}\left(\frac{\exp \left(X_{t}^{\prime} \hat{\beta}\right) \psi_{s}(t / T)}{1+\exp \left(X_{t}^{\prime} \hat{\beta}\right)}\right)=\sum_{t=1}^{n} \pi(t \mid t) \psi_{s}(t / T)
\end{aligned}
$$




\subsection{Aplicação do Modelo com Ondaletas em Exem- plo do Artigo Shumway e Stoffer (1991)}

Shumway e Stoffer (1991) apresentaram um exemplo de modelos com mudança de regime na forma dinâmica de espaço de estados.

O problema consiste em detectar um grande número de movimentos de alvos $\left(\theta_{t}\right)$ usando um vetor de sensores $\left(y_{t}\right)$. Não se conhece, em dado tempo, qual alvo foi detectado por determinado sensor. Utilizaram o modelo na forma de espaço de estados (5.1) onde $y_{t}$ é um vetor de sensores, $\theta_{t}$ o vetor de alvos e $F_{t}$ a matriz do sistema que captará as mudanças de posição.

Suponha um vetor $(3 \times 1)$ de um satélite $y_{t}=\left(y_{t_{1}}, y_{t_{2}}, y_{t_{3}}\right)^{\prime}$ que está observando uma combinação de sinais (ou alvos) $\theta_{t}^{\prime}=\left(\theta_{t_{1}}, \theta_{t_{2}}, \theta_{t_{3}}\right)$. Por exemplo, para uma matriz do sistema dada por

$$
F_{t}=\left(\begin{array}{lll}
1 & 0 & 0 \\
1 & 0 & 0 \\
1 & 0 & 0
\end{array}\right),
$$

interpretamos que todos os sensores detectaram o sinal $\theta_{t_{1}}$ e para a matriz

$$
F_{t}=\left(\begin{array}{lll}
0 & 1 & 0 \\
1 & 0 & 0 \\
0 & 0 & 1
\end{array}\right)
$$

interpretamos que o primeiro sensor $\left(y_{t_{1}}\right)$ observou o segundo alvo $\left(\theta_{t_{2}}\right)$, o segundo sensor $\left(y_{t_{2}}\right)$ observou o primeiro alvo $\left(\theta_{t_{1}}\right)$ e o terceiro sensor $\left(y_{t_{3}}\right)$ observou o terceiro alvo $\left(\theta_{t_{3}}\right)$.

Para o exemplo, Shumway e Stoffer (1991) consideraram $M=10$ possíveis valores para a matriz $F_{t}$. A Tabela 5.1 apresenta as matrizes, $M_{1}, M_{2}, \ldots, M_{10}$, que são formadas a partir dos alvos detectados por cada sensor. Note que as matrizes exemplificadas acima são identificadas como $M_{1}$ e $M_{7}$ respectivamente.

Simulamos no programa S-Plus o modelo na forma de espaço de estados

$$
\begin{aligned}
& y_{t}=F_{t} \theta_{t}+v_{t} \mathrm{e} \\
& \theta_{t}=G_{t} \theta_{t-1}+w_{t},
\end{aligned}
$$

supondo 
Tabela 5.1: Valores de $F_{t}$ para o exemplo Shumway e Stoffer (1991).

\begin{tabular}{c|ccc}
\hline \hline & & & \\
Matriz & $y_{t_{1}}$ & $y_{t_{2}}$ & $y_{t_{3}}$ \\
\hline \hline & & & \\
$M_{1}$ & $\theta_{t_{1}}$ & $\theta_{t_{1}}$ & $\theta_{t_{1}}$ \\
$M_{2}$ & $\theta_{t_{1}}$ & $\theta_{t_{1}}$ & $\theta_{t_{2}}$ \\
$M_{3}$ & $\theta_{t_{1}}$ & $\theta_{t_{2}}$ & $\theta_{t_{1}}$ \\
$M_{4}$ & $\theta_{t_{2}}$ & $\theta_{t_{1}}$ & $\theta_{t_{1}}$ \\
$M_{5}$ & $\theta_{t_{1}}$ & $\theta_{t_{3}}$ & $\theta_{t_{2}}$ \\
$M_{6}$ & $\theta_{t_{1}}$ & $\theta_{t_{2}}$ & $\theta_{t_{3}}$ \\
$M_{7}$ & $\theta_{t_{2}}$ & $\theta_{t_{1}}$ & $\theta_{t_{3}}$ \\
$M_{8}$ & $\theta_{t_{2}}$ & $\theta_{t_{3}}$ & $\theta_{t_{1}}$ \\
$M_{9}$ & $\theta_{t_{3}}$ & $\theta_{t_{2}}$ & $\theta_{t_{1}}$ \\
$M_{10}$ & $\theta_{t_{3}}$ & $\theta_{t_{1}}$ & $\theta_{t_{2}}$ \\
& & & \\
\hline \hline
\end{tabular}

i) $v_{t} \sim$ iid $N(0, V)$;

ii) $w_{t} \sim$ iid $N(0, W)$;

iii) $v_{t}$ independente de $w_{t}$;

iv) $\theta_{0} \sim N(\mu, \Sigma)$;

com

$$
G_{t}=\left(\begin{array}{ccc}
1.005 & 0 & 0 \\
0 & 0.990 & 0 \\
0 & 0 & 1.00
\end{array}\right),
$$

que corresponde a um passeio aleatório com um pequeno acréscimo e decréscimo ao longo do tempo;

$$
V=\left(\begin{array}{ccc}
0.0625 & 0 & 0 \\
0 & 0.0625 & 0 \\
0 & 0 & 0.0625
\end{array}\right)
$$




$$
\begin{gathered}
W=\left(\begin{array}{ccc}
0.0025 & 0 & 0 \\
0 & 0.0025 & 0 \\
0 & 0 & 0.0025
\end{array}\right) ; \\
\mu=\left(\begin{array}{llll}
5 & 5 & 5)^{\prime} & \text { e } n=100 .
\end{array}\right.
\end{gathered}
$$

Em cada exemplo, utilizamos somente dois dos 10 possíveis valores da matriz $F_{t}$. A Tabela 5.2 resume as informações sobre $F_{t}$ para os três exemplos considerados:

\begin{tabular}{|c|c|}
\hline Exemplos & $F_{t}$ \\
\hline Exemplo 1 & $\begin{array}{c}M_{6}, \quad 1 \leq t \leq 49 \\
M_{7}, \quad 50 \leq t \leq 100\end{array}$ \\
\hline Exemplo 2 & $\begin{array}{c}M_{5}, \quad 1 \leq t \leq 30 \text { e } 50 \leq t \leq 69 \\
M_{9}, \quad 31 \leq t \leq 49 \text { e } 70 \leq t \leq 100\end{array}$ \\
\hline Exemplo 3 & $\begin{array}{c}M_{1}, \quad 1 \leq t \leq 30 \text { e } 50 \leq t \leq 69 \\
M_{9}, \quad 31 \leq t \leq 49 \text { e } 70 \leq t \leq 100\end{array}$ \\
\hline
\end{tabular}

Tabela 5.2: Valores de $F_{t}$ para os exemplos considerados.

Implementamos na linguagem Fortran 90 um programa que calcula as estimativas dos parâmetros a partir do algoritmo EM e calcula o Filtro de Kalman no processo iterativo para estimar o estado. As equações utilizadas para os estimadores foram deduzidas na seção (5.3).

Analisamos a estimação de cinco (5) modelos para cada exemplo: o modelo de Shumway e Stoffer (1991), que considera as probabilidades associadas às 
matrizes $F_{t}$ utilizadas em cada exemplo como 0.5 e os quatro (4) outros que utilizam as ondaletas Haar, Chapéu Mexicano, Morlet e Shannon para modelar estas probabilidades.

Para todos os modelos com ondaletas, expressamos as probabilidades como

$$
\pi(t)=\frac{\exp \left(\sum_{l=1}^{s} \beta_{l} \psi_{l}(t / T)\right)}{1+\exp \left(\sum_{l=1}^{s} \beta_{l} \psi_{l}(t / T)\right)} .
$$

Determinamos que $s=3$, isto é, consideramos as três primeiras ondaletas com fator de escala $j=1,2$ e fator de translação $k=1, \ldots, 2^{j-1}$. Escolhemos este valor para $s$ depois de alguns testes com outros valores. O que observamos é que não seriam necessárias muitas ondaletas para cada probabilidade.

Para chegarmos às estimativas da probabilidades $\pi(t)$, tivemos que resolver um sistema não-linear com $s=3$ equações dado pelas equações em (5.12). Utilizamos a subrotina "newt", p. 379 de Press, Teukolsky, Vetterling e Flannery (1992) que encontra raízes de um sistema de equações não-lineares.

Apresentamos a seguir alguns comentários sobre os exemplos. 
Exemplo 1:

Todos os modelos apresentaram boas estimativas das séries (Figura 5.1). Em relação aos estados, o modelo que utilizou a ondaleta Chapéu Mexicano não estimou bem $\theta_{t_{1}}$ e $\theta_{t_{2}}$ (Figura 5.2).

Da Tabela 5.3 observamos que o modelo que apresentou maior valor da função log-verossimilhança foi o que utilizou a ondaleta Shannon. O modelo Haar apresentou coeficientes estimados das ondaletas muito pequenos (próximo de zero) e o modelo Chapéu Mexicano, coeficientes muito grandes. As demais estimativas estão razoáveis.
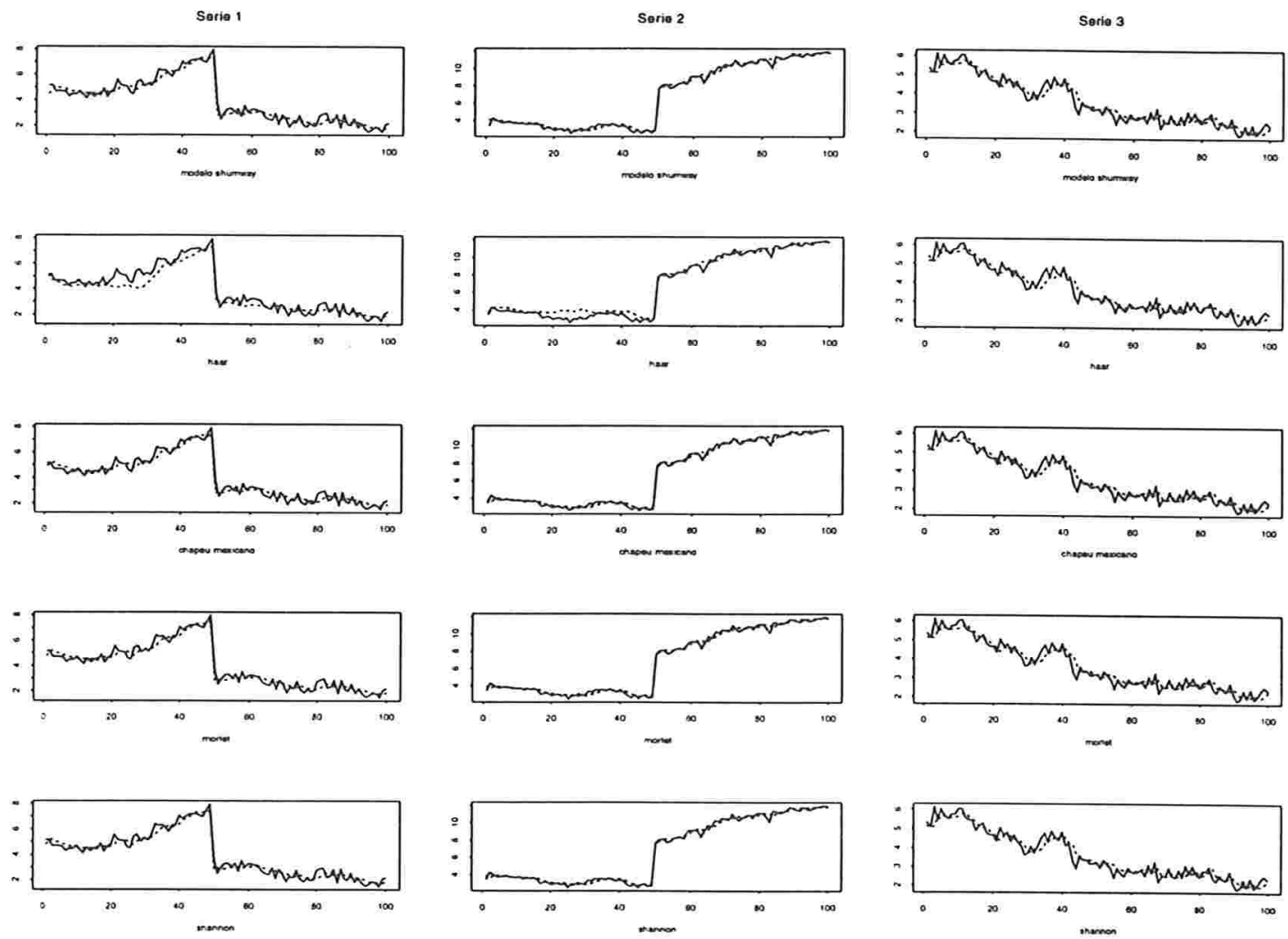

Figura 5.1: Gráficos das séries $y_{t_{1}}, y_{t_{2}}$ e $y_{t_{3}}$ e suas respectivas estimativas para os modelos Shumway e Stoffer (1991), ondaletas Haar, Chapéu Mexicano, Morlet e Shannon (Exemplo 1). 
Estado 1
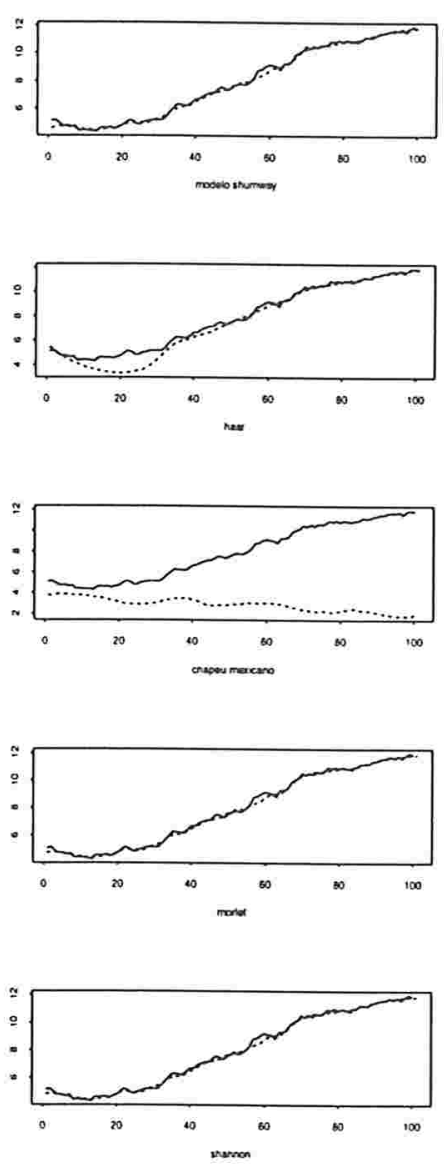

Estado 2
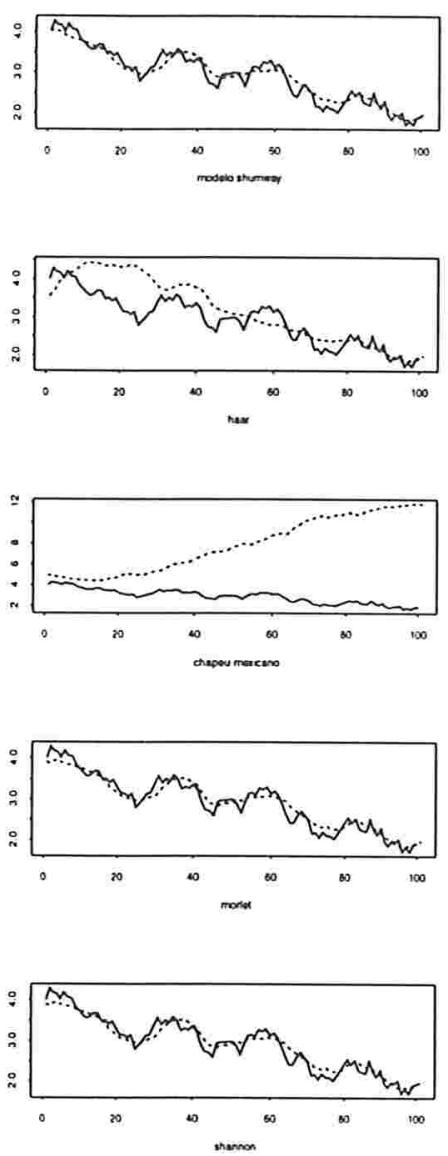

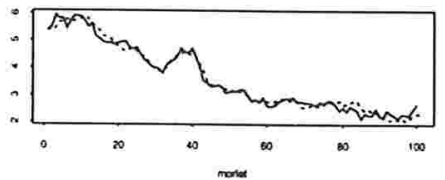

Estado 3
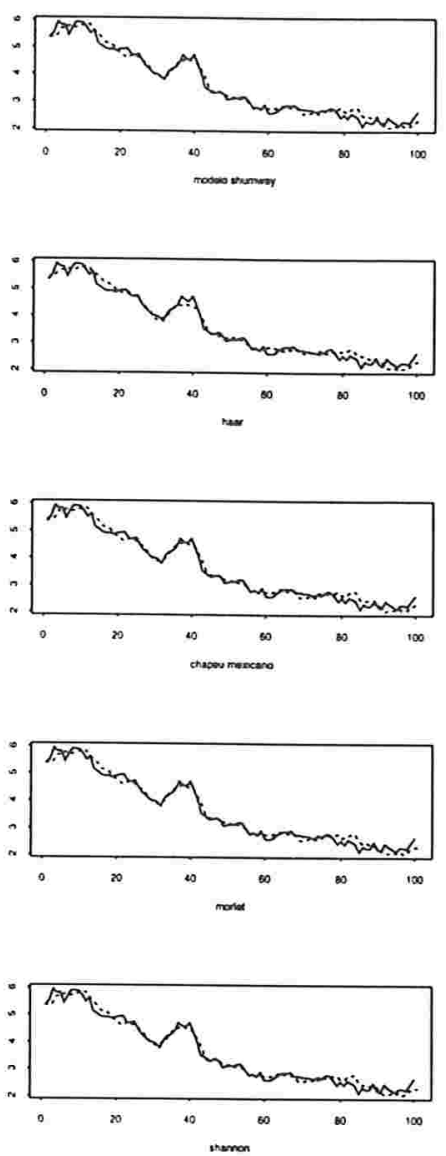

Figura 5.2: Gráficos dos estados $\theta_{t_{1}}, \theta_{t_{2}}$ e $\theta_{t_{3}}$ e suas respectivas estimativas para os modelos Shumway e Stoffer (1991), ondaletas Haar, Chapéu Mexicano, Morlet e Shannon (Exemplo 1).

A Figura 5.3 apresenta as estimativas das probabilidades e probabilidades filtradas. Em relação às probabilidades filtradas, só existe diferença no modelo Chapéu Mexicano que inverte o sentido das probabilidades e no modelo Haar que apresenta problemas de estimação dos primeiros 20 valores. Notamos ainda que os modelos Morlet e Shannon captaram as diferenças de uso das matrizes $M_{6}$ e $M_{7}$ manifestados em $P 6(t)$ e $P 7(t)$. 
$P G(t)$
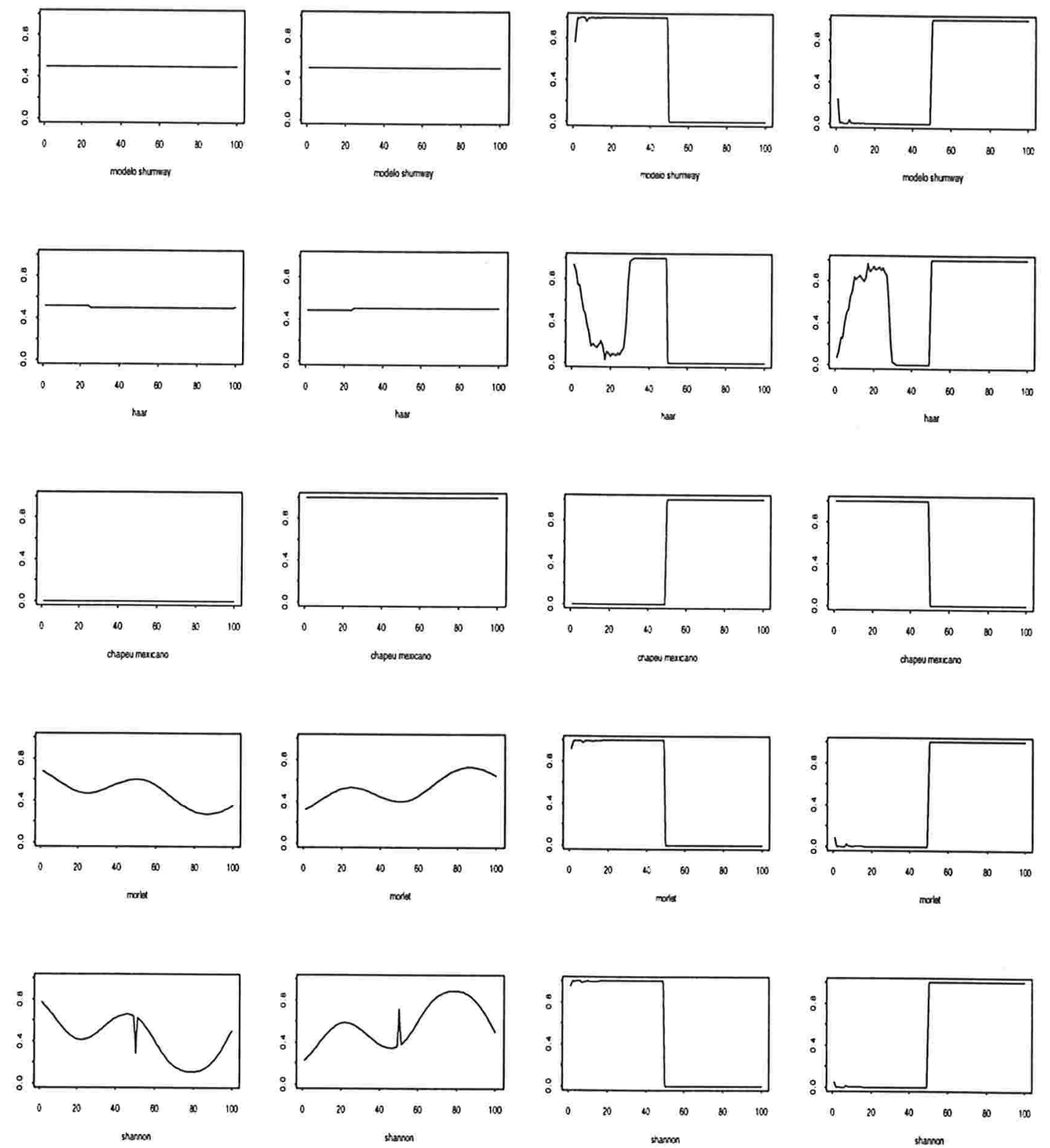

Figura 5.3: Gráficos das estimativas das probabilidades $P 6(t)$ e $P 7(t)$ e das probabilidades filtradas $P 6(t \mid t)$ e $P 7(t \mid t)$ para os modelos Shumway e Stoffer (1991), ondaletas Haar, Chapéu Mexicano, Morlet e Shannon (Exemplo 1). 
Tabela 5.3: Resultados obtidos para o exemplo 1 utilizando os modelos Shumway e Stoffer (1991), ondaletas Haar, Chapéu Mexicano, Morlet e Shannon, respectivamente. (*) Modelo com log verossimilhança maior.

\begin{tabular}{c||c|c|c|c|c}
\hline \hline Parâmetros & Shumway & Haar & Chapéu & Morlet & Shannon \\
\hline \hline Iteração $_{\text {log veros }}$ & 589 & 219 & 133 & 183 & 150 \\
& -101.3268 & -150.5020 & -518.6409 & -89.6873 & $-80.7094\left(^{*}\right)$ \\
\hline \hline & & & & & \\
$G_{11}$ & 0.9979 & 0.9999 & 0.9725 & 0.9976 & 0.9983 \\
$G_{22}$ & 0.9821 & 0.8991 & 0.9968 & 0.9817 & 0.9769 \\
$G_{33}$ & 0.9360 & 1.0380 & 0.9497 & 0.9471 & 0.9411 \\
& & & & & \\
\hline \hline & & & & & \\
$V_{11}$ & 0.1075 & 0.2426 & 0.0955 & 0.1015 & 0.0997 \\
$V_{22}$ & 0.0606 & 0.1916 & 0.0478 & 0.0547 & 0.0538 \\
$V_{33}$ & 0.0602 & 0.0682 & 0.0609 & 0.0293 & 0.0600 \\
& & & & & \\
\hline \hline & & & & & \\
$W_{11}$ & 0.0147 & 0.0122 & 0.0155 & 0.0187 & 0.0191 \\
$W_{22}$ & 0.0125 & 0.0080 & 0.0253 & 0.0133 & 0.0142 \\
$W_{33}$ & 0.0303 & 0.0225 & 0.0298 & 0.0293 & 0.0310 \\
$\beta_{3}$ & & & & & \\
$\beta_{3}$ & - & 0.0206 & -69.9560 & 0.6694 & 1.4428 \\
\hline \hline
\end{tabular}


Exemplo 2:

A Tabela 5.4 apresenta os resultados para os modelos analisados. O modelo com maior log da verossimilhança foi o que utilizou a ondaleta Morlet. Da mesma forma que no exemplo anterior, os coeficientes estimados para as ondaletas Haar e Chapéu Mexicano apresentam problemas (na Haar os coeficientes são muito pequenos e no Chapéu Mexicano muito grandes). O modelo Haar difere pouco do modelo de Shumway e Stoffer (1991) por causa dos valores estimados dos coeficientes.

As Figuras 5.4 e 5.5 mostram as estimativas das séries e estados respectivamente para os modelos analisados. As estimativas são muito boas a menos do modelo Chapéu Mexicano.
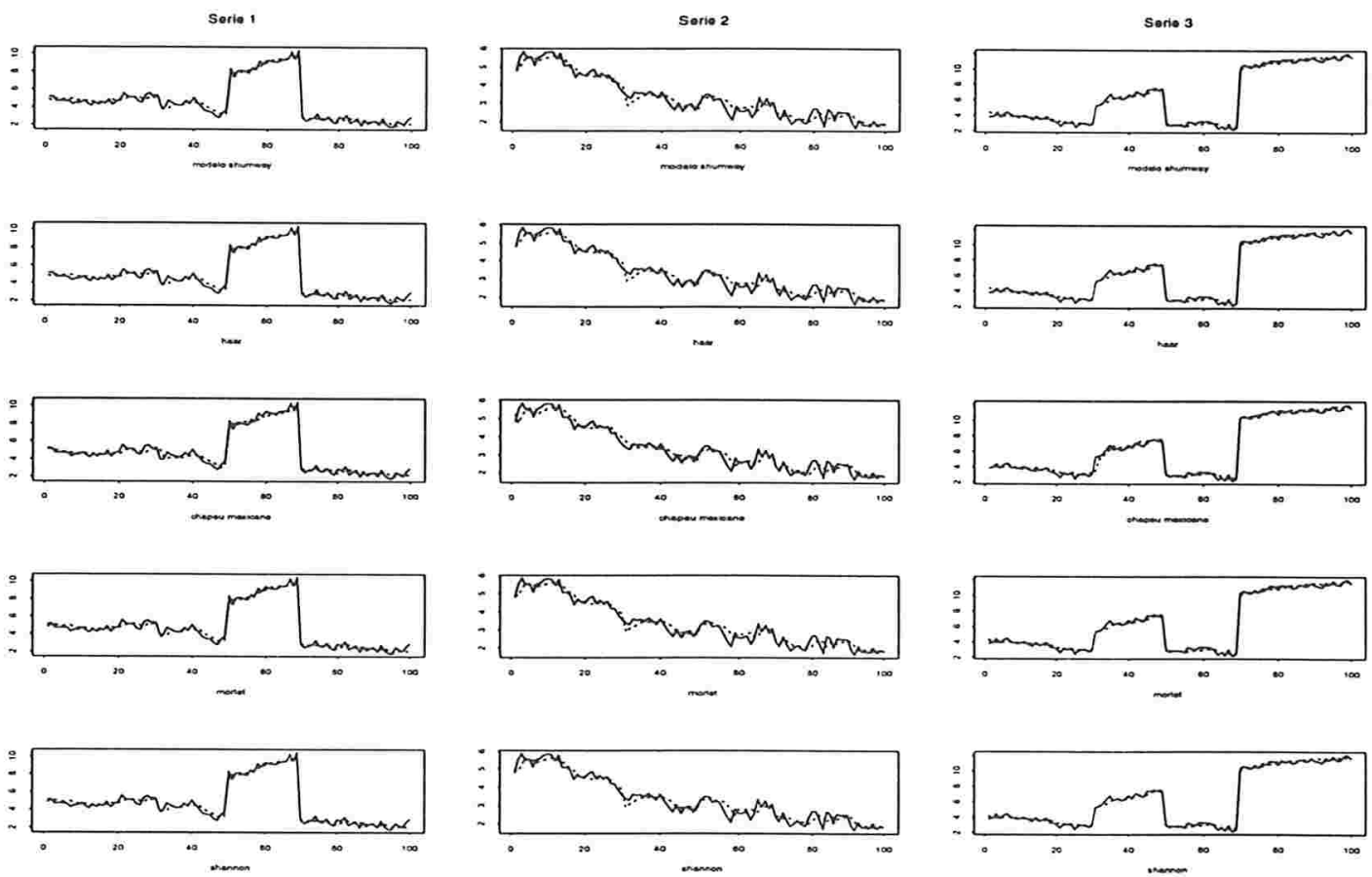

Figura 5.4: Gráficos das séries $y_{t_{1}}, y_{t_{2}}$ e $y_{t_{3}}$ e suas respectivas estimativas para os modelos Shumway e Stoffer (1991), ondaletas Haar, Chapéu Mexicano, Morlet e Shannon (Exemplo 2). 

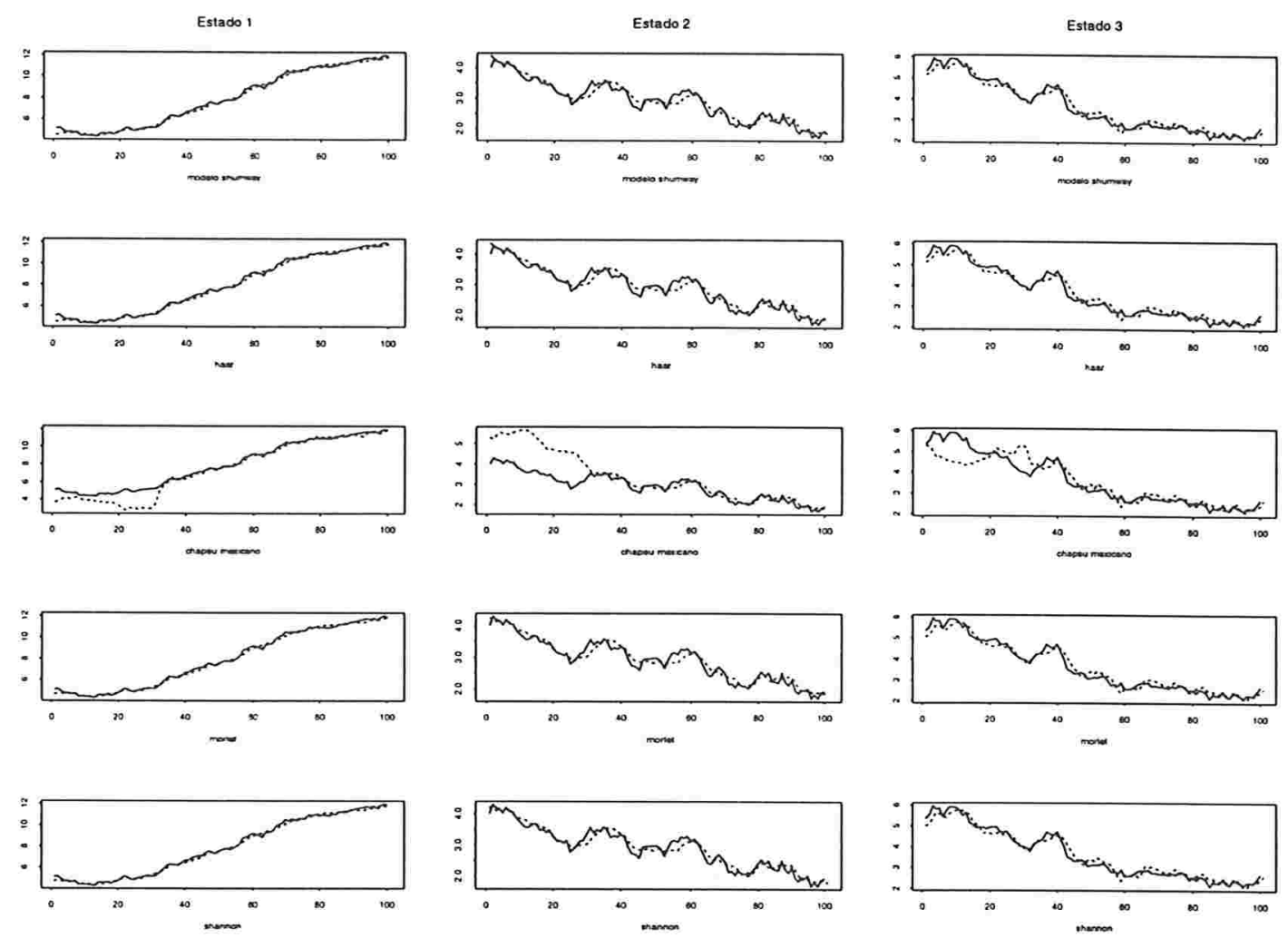

Figura 5.5: Gráficos dos estados $\theta_{t_{1}}, \theta_{t_{2}}$ e $\theta_{t_{3}}$ e suas respectivas estimativas para os modelos Shumway e Stoffer (1991), ondaletas Haar, Chapéu Mexicano, Morlet e Shannon (Exemplo 2).

A Figura 5.6 apresenta as estimativas das probabilidades filtradas para os modelos considerados. Também aqui observamos problemas no modelo que utilizou a ondaleta Chapéu Mexicano. 
P5(I)

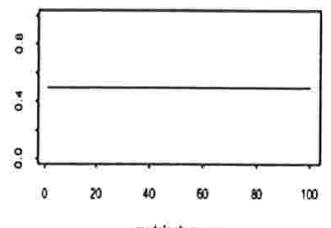

modeb ithnsasy
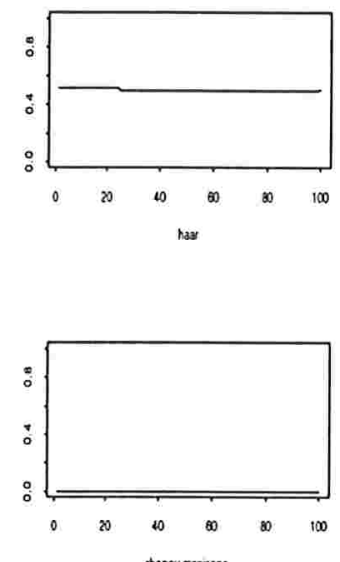

chapsu nerciano
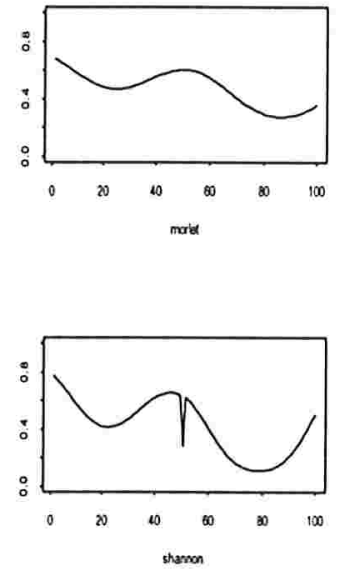

P9(II)
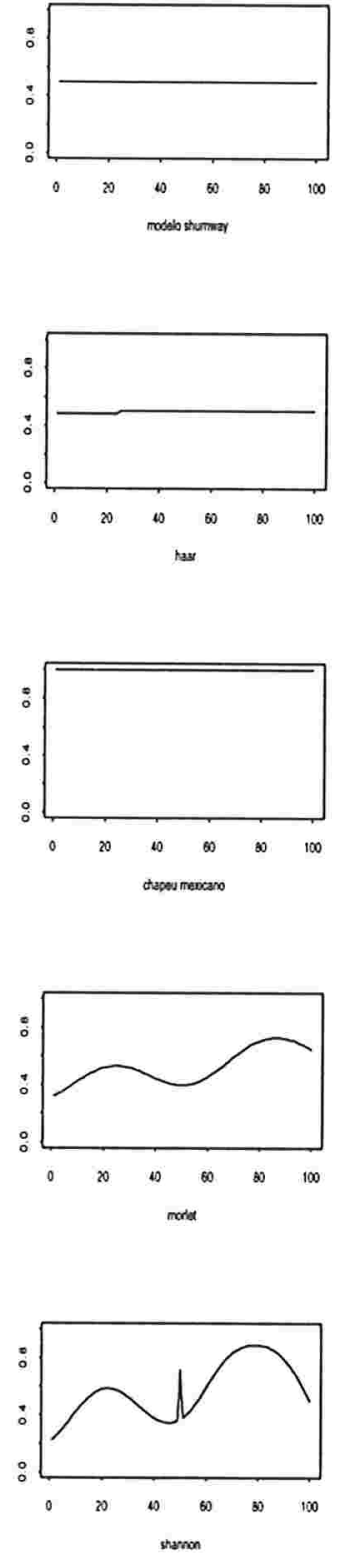

$P 5($ (Ii)
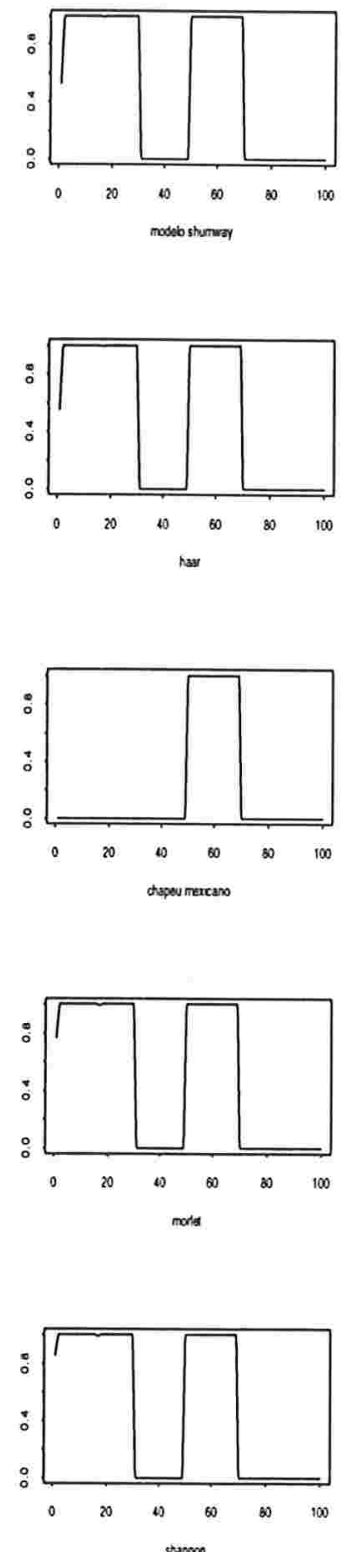
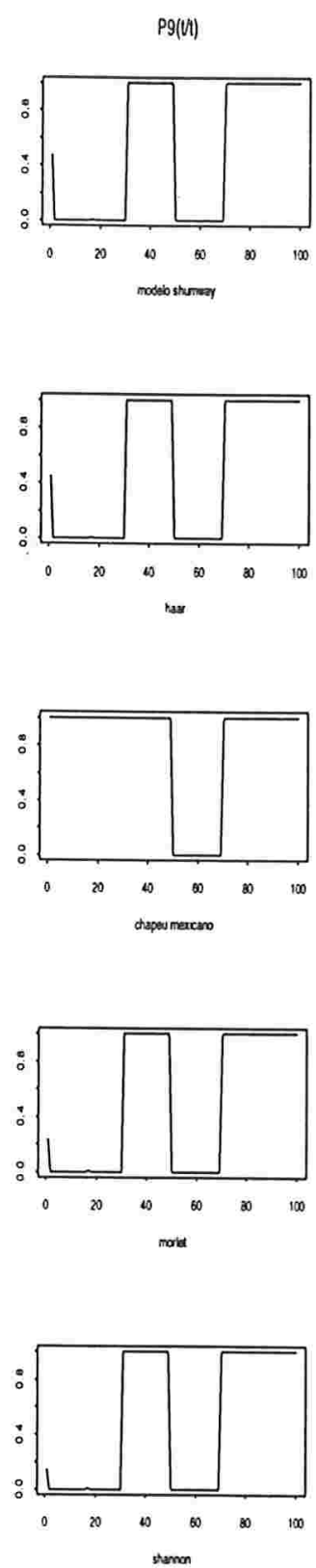

Figura 5.6: Gráficos das estimativas das probabilidades $P 5(t)$ e $P 9(t)$ e das probabilidades filtradas $P 5(t \mid t)$ e $P 9(t \mid t)$ para os modelos Shumway e Stoffer (1991), ondaletas Haar, Chapéu Mexicano, Morlet e Shannon (Exemplo 2). 
Tabela 5.4: Resultados obtidos para o exemplo 2 utilizando os modelos Shumway e Stoffer (1991), ondaletas Haar, Chapéu Mexicano, Morlet e Shannon, respectivamente. $\left({ }^{*}\right)$ Modelo com log verossimilhança maior.

\begin{tabular}{|c|c|c|c|c|c|}
\hline Parâmetros & Shumway & Haar & Chapéu & Morlet & Shannon \\
\hline $\begin{array}{l}\text { Iteração } \\
\log \text { veros }\end{array}$ & $\begin{array}{c}51 \\
-98.4054\end{array}$ & $\begin{array}{c}51 \\
-97.4826\end{array}$ & $\begin{array}{c}51 \\
-286.2793\end{array}$ & $\begin{array}{c}51 \\
-88.1812(*)\end{array}$ & $\begin{array}{c}51 \\
-95.4079\end{array}$ \\
\hline $\begin{array}{l}G_{11} \\
G_{22} \\
G_{33}\end{array}$ & $\begin{array}{l}1.0035 \\
0.9376 \\
0.8732\end{array}$ & $\begin{array}{l}1.0036 \\
0.9380 \\
0.8729\end{array}$ & $\begin{array}{l}0.9979 \\
0.9826 \\
0.8954\end{array}$ & $\begin{array}{l}1.0043 \\
0.9401 \\
0.8686\end{array}$ & $\begin{array}{l}1.0046 \\
0.9398 \\
0.8655\end{array}$ \\
\hline $\begin{array}{l}V_{11} \\
V_{22} \\
V_{33}\end{array}$ & $\begin{array}{l}0.0930 \\
0.0487 \\
0.0685\end{array}$ & $\begin{array}{l}0.0927 \\
0.0487 \\
0.0683\end{array}$ & $\begin{array}{l}0.0843 \\
0.0485 \\
0.0565\end{array}$ & $\begin{array}{l}0.0887 \\
0.0473 \\
0.0662\end{array}$ & $\begin{array}{l}0.0866 \\
0.0460 \\
0.0646\end{array}$ \\
\hline $\begin{array}{l}W_{11} \\
W_{22} \\
W_{33}\end{array}$ & $\begin{array}{l}0.0164 \\
0.0184 \\
0.0387\end{array}$ & $\begin{array}{l}0.0166 \\
0.0184 \\
0.0389\end{array}$ & $\begin{array}{l}0.0771 \\
0.0225 \\
0.0463\end{array}$ & $\begin{array}{l}0.0185 \\
0.0189 \\
0.0419\end{array}$ & $\begin{array}{l}0.0198 \\
0.0194 \\
0.0440\end{array}$ \\
\hline $\begin{array}{l}\beta_{1} \\
\beta_{2} \\
\beta_{3}\end{array}$ & $\begin{array}{l}- \\
- \\
-\end{array}$ & $\begin{array}{c}0.0206 \\
0.0291 \\
0\end{array}$ & $\begin{array}{c}-69.9560 \\
11.8477 \\
22.4574\end{array}$ & $\begin{array}{l}0.6694 \\
0.3199 \\
0.5935\end{array}$ & $\begin{array}{c}1.4428 \\
-0.0876 \\
1.0337\end{array}$ \\
\hline
\end{tabular}


Exemplo 3:

Para os dados deste exemplo não conseguimos estimar a matriz de covariância $W$ para nenhum dos modelos. Assim, fixamos a matriz $W$ como diagonal, cujos valores na diagonal são 0.1 e estimamos os demais parâmetros.

Da Tabela 5.5 observamos que o modelo com maior log da verossimilhança foi o que utilizou a ondaleta Haar. E para todos os modelos as estimativas dos parâmetros diferiam na quinta ou sexta casa decimal.

As Figuras 5.7 e 5.8 apresentam as estimativas das séries e dos estados respectivamente para todos os modelos. Observamos o mesmo comportamento para todos os modelos.
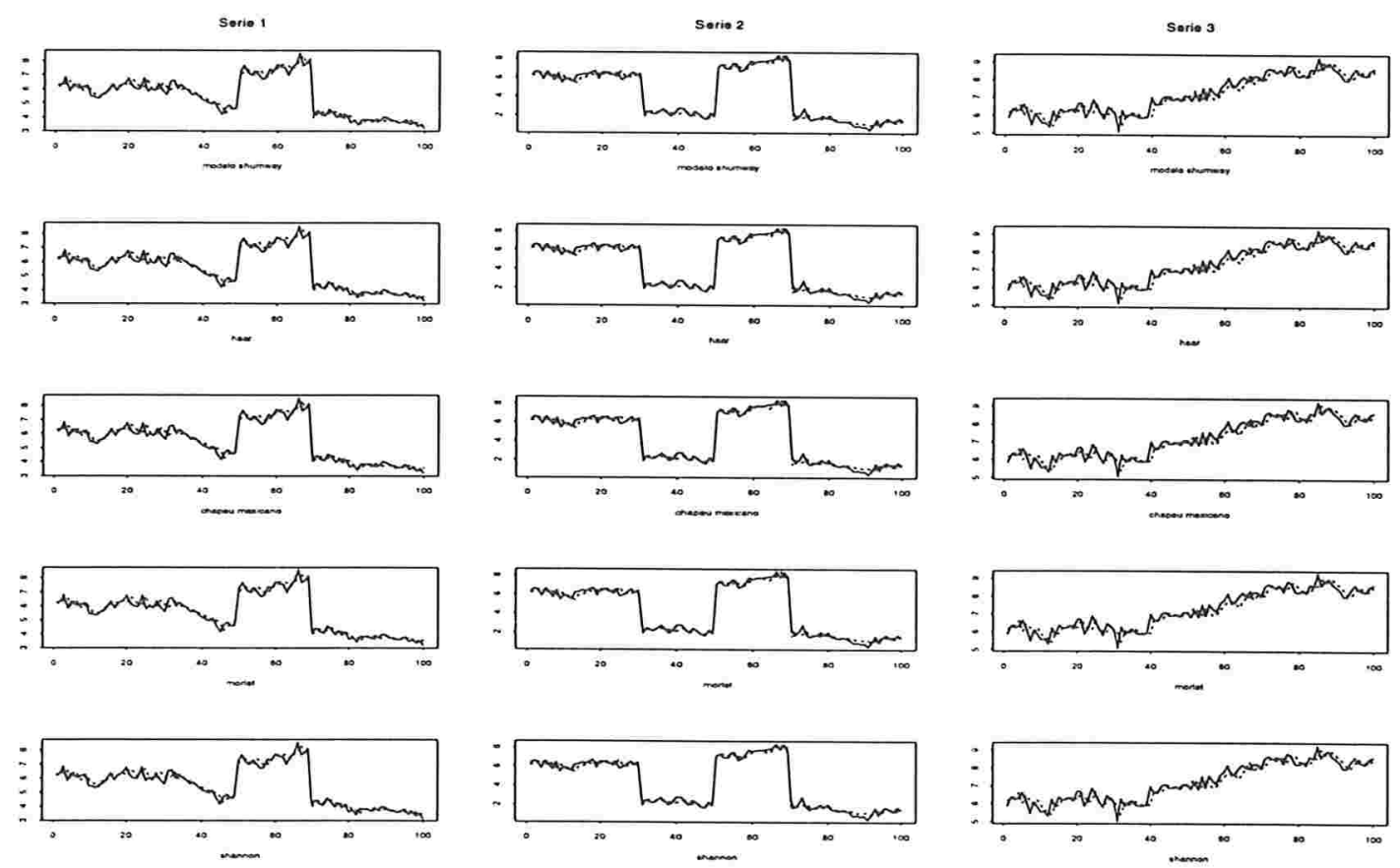

Figura 5.7: Gráficos das séries $y_{t_{1}}, y_{t_{2}}$ e $y_{t_{3}}$ e suas respectivas estimativas para os modelos Shumway e Stoffer (1991), ondaletas Haar, Chapéu Mexicano, Morlet e Shannon (Exemplo 3). 

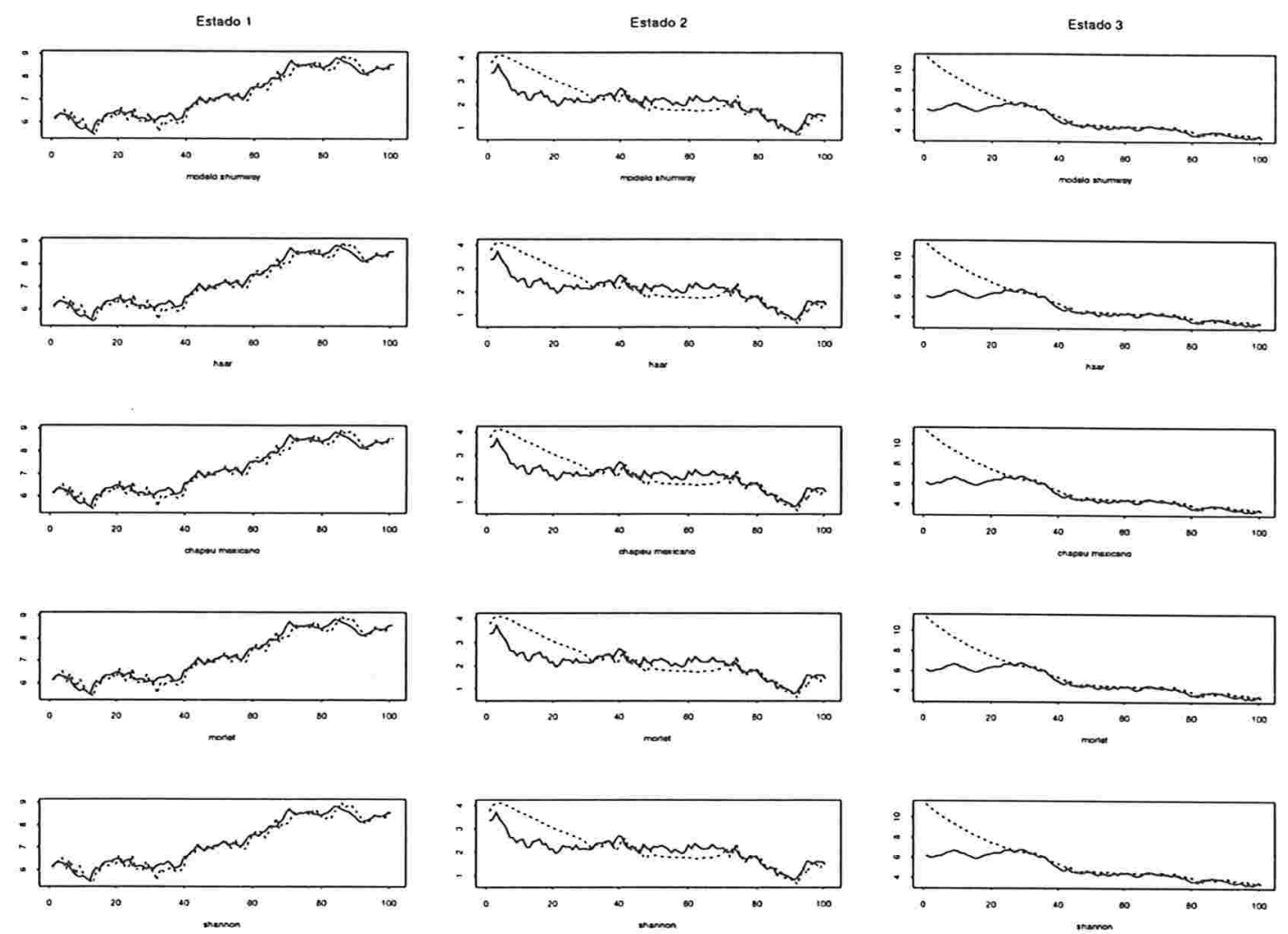

Figura 5.8: Gráficos dos estados $\theta_{t_{1}}, \theta_{t_{2}}$ e $\theta_{t_{3}}$ e suas respectivas estimativas para os modelos Shumway e Stoffer (1991), ondaletas Haar, Chapéu Mexicano, Morlet e Shannon (Exemplo 3).

A Figura 5.9 apresenta as estimativas das probabilidades e probabilidades filtradas relativas ao exemplo. Observamos que todos os modelos que utilizam ondaletas acompanham as mudanças da matriz $F_{t}$ expressas nas estimativas de $P 1(t)$ e $P 9(t)$. Para todos os modelos encontramos as mesmas estimativas para as probabilidades filtradas. 
P1(t)

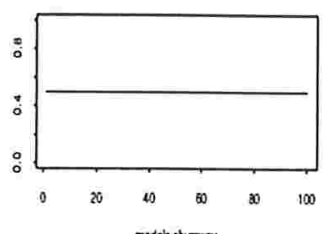

nodeb stumay
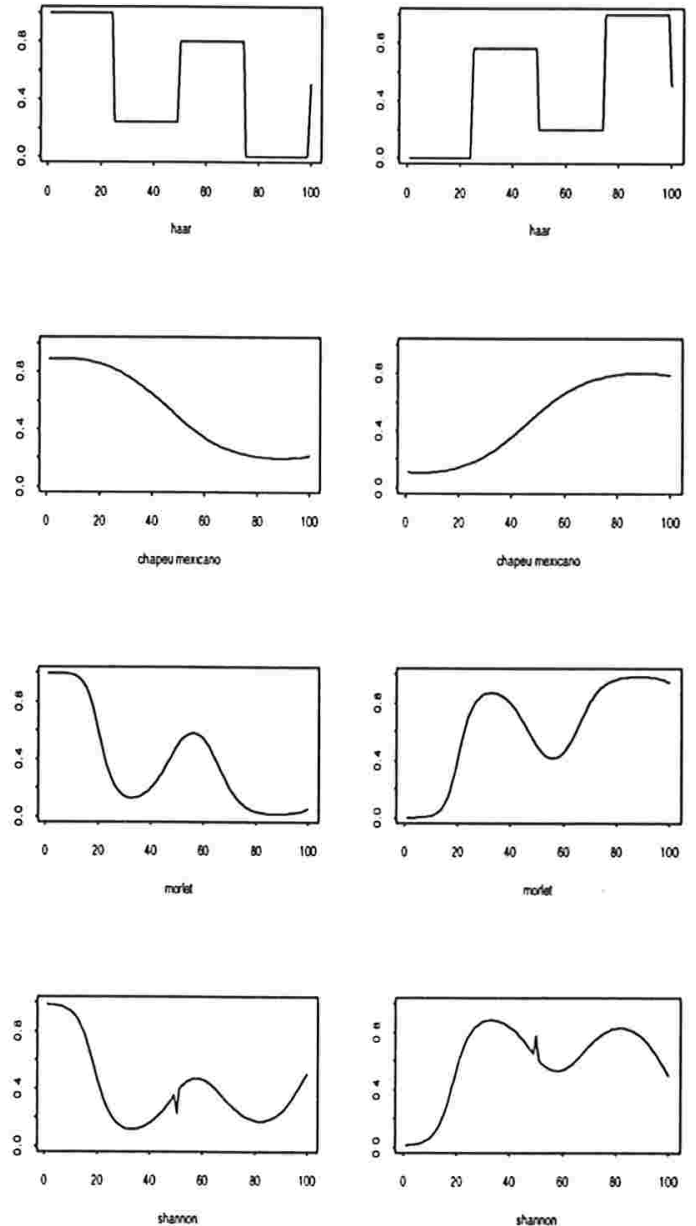

$P 9(1)$
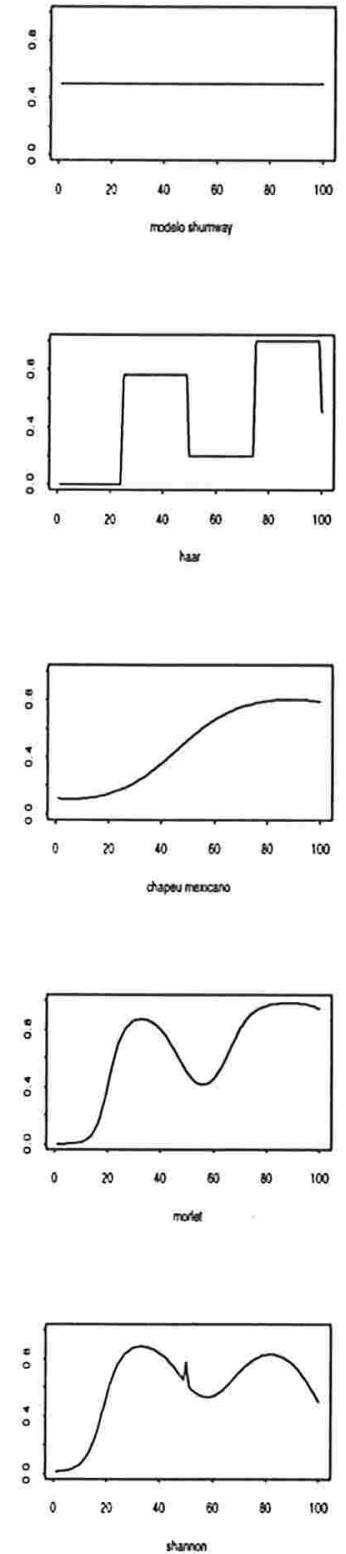
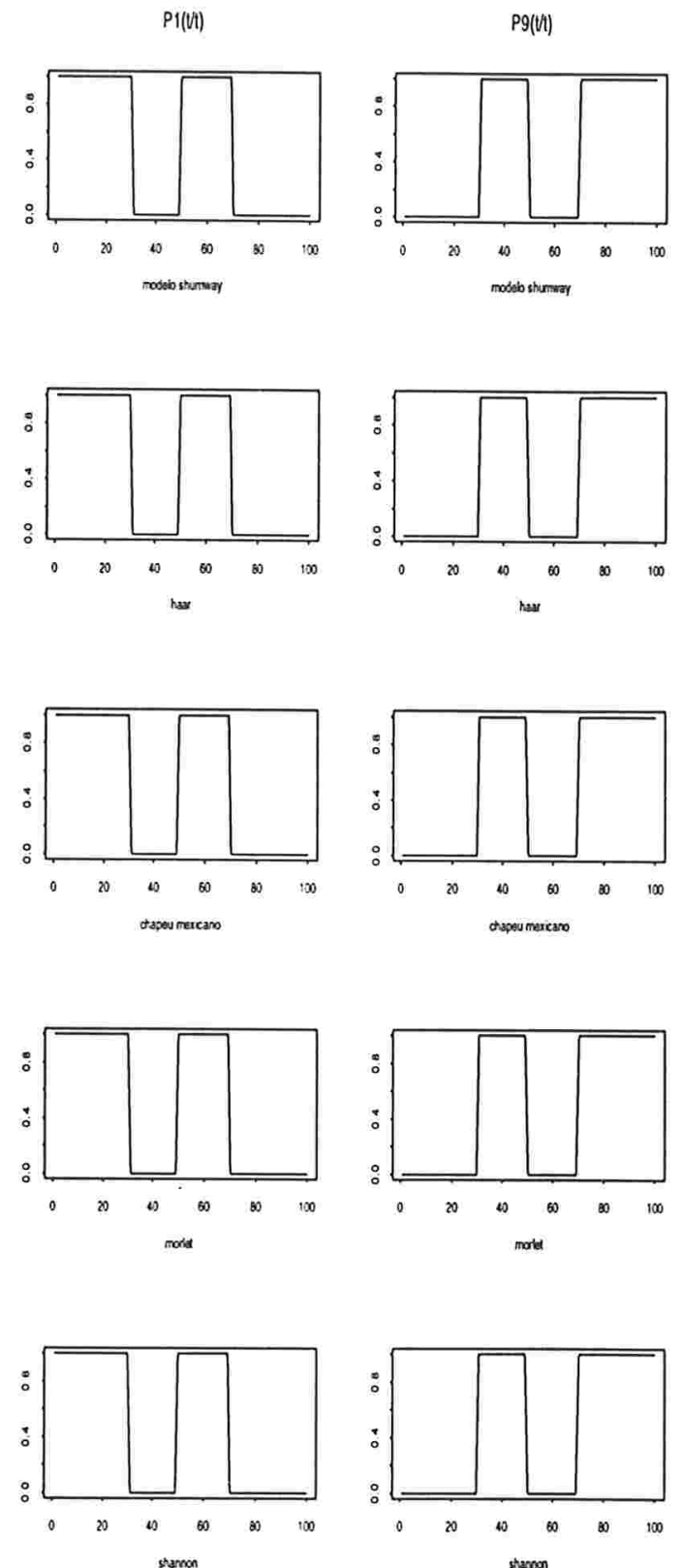

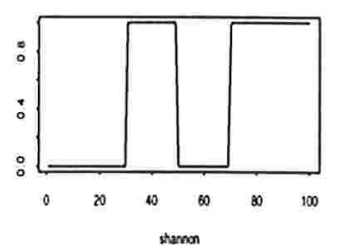

Figura 5.9: Gráficos das estimativas das probabilidades $P 1(t)$ e $P 9(t)$ e das probabilidades filtradas $P 1(t \mid t)$ e $P 9(t \mid t)$ para os modelos Shumway e Stoffer (1991), ondaletas Haar, Chapéu Mexicano, Morlet e Shannon (Exemplo 3). 
Tabela 5.5: Resultados obtidos para o exemplo 3 utilizando os modelos Shumway e Stoffer (1991), ondaletas Haar, Chapéu Mexicano, Morlet e Shannon, respectivamente. (*) Modelo com log verossimilhança maior.

\begin{tabular}{c||c|c|c|c|c}
\hline \hline Parâmetros & Shumway & Haar & Chapéu & Morlet & Shannon \\
\hline \hline Iteração & 51 & 51 & 51 & 51 & 51 \\
log veros & -103.8511 & $-61.5200\left(^{*}\right)$ & -87.4213 & -72.7203 & -85.6158 \\
\hline \hline & & & & & \\
$G_{11}$ & 1.0023 & 1.0023 & 1.0023 & 1.0023 & 1.0023 \\
$G_{22}$ & 0.6204 & 0.6204 & 0.6204 & 0.6204 & 0.6204 \\
$G_{33}$ & 0.9331 & 0.9331 & 0.9331 & 0.9331 & 0.9331 \\
& & & & & \\
\hline \hline & & & & & \\
$V_{11}$ & 0.0369 & 0.0369 & 0.0369 & 0.0369 & 0.0369 \\
$V_{22}$ & 0.0519 & 0.0519 & 0.0519 & 0.0519 & 0.0519 \\
$V_{33}$ & 0.0564 & 0.0564 & 0.0564 & 0.0564 & 0.0564 \\
& & & & & \\
\hline \hline$W_{11}$ & - & - & - & - & - \\
$W_{22}$ & - & - & - & - & - \\
$W_{33}$ & - & - & -1.1618 & 3.7146 & 1.9011 \\
$\beta_{3}$ & - & 3.9090 & 0.4908 & 2.3323 & 0.4908 \\
$\beta_{1}$ & - & & & & \\
\hline \hline
\end{tabular}




\section{Capítulo 6}

\section{Modelos com Ondaletas na Matriz do Sistema}

\subsection{Introdução}

Como vimos no capítulo anterior, as séries temporais com mudança de regime podem ser colocadas na forma de espaço de estados, dentro da estrutura de modelos lineares dinâmicos.

Shumway e Stoffer (1991) utilizaram um modelo na forma de espaço de estados cuja matriz do sistema $F_{t}$ varia no tempo de acordo com um processo aleatório. A incorporação da estrutura de variação ("switching") na matriz $F_{t}$ acontece supondo que existem $M$ configurações possíveis que são estados de um processo não-estacionário independente definido por um conjunto de probabilidades. No capítulo anterior utilizamos ondaletas no conjunto de probabilidades supondo variação ao longo do tempo.

Neste capítulo propomos uma metodologia alternativa para séries com mudança de regime. Utilizamos os modelos na forma de espaço de estados e consideramos que a matriz do sistema $F_{t}$ pode incorporar a estrutura de variação ("switching") sendo modelada por uma soma de ondaletas.

A proxima seção apresenta a formulação do modelo proposto. Utilizamos também o algoritmo EM e o Filtro de Kalman para obter estimadores dos parâmetros e do vetor de estado, respectivamente. Os estimadores obtidos são descritos na seção 6.3. Encontramos problemas na estimação de parâmetros de variância e a seção 6.4 trata deste problema. 


\subsection{Formulação do Modelo}

Seja o seguinte modelo na forma de espaço de estados:

$$
\begin{aligned}
y_{t} & =F_{t} \theta_{t}+v_{t} \\
\theta_{t} & =G_{t} \theta_{t-1}+w_{t} \quad \mathrm{e} \\
F_{t}^{(l, m)} & =\sum_{j, k} \gamma_{j k}^{(l, m)} \psi_{j, k}(t / T)
\end{aligned}
$$

onde

$y_{t}$ é o vetor de observações $(r \times 1), t=1, \ldots, n$;

$F_{t}$ é a matriz do sistema $(r \times p)$;

$\theta_{t}$ é o vetor de estado $(p \times 1)$;

$G_{t}$ é a matriz de transição $(p \times p)$;

$(l, m)$ denota o elemento $(l, m)$ da matriz $F_{t}$;

$\gamma_{j k}$ são parâmetros associados às ondaletas da matriz $F_{t}$;

$\psi_{j, k}(t / T)$ é a ondaleta no tempo $(t / T)$, fator de escala j e fator de translação $\mathrm{k}, \operatorname{com} T=n$;

$v_{t}$ é o vetor de erros associado às observações $(r \times 1)$;

$w_{t}$ é o vetor de erros associado ao estado $(p \times 1)$;

Sejam as seguintes hipóteses associadas ao modelo:

i) $v_{t} \sim$ iid $N\left(0, V_{t}\right)$;

ii) $w_{t} \sim$ iid $N\left(0, W_{t}\right)$;

iii) $v_{t}$ independente de $w_{t}$;

iv) $\left(\theta_{0} \mid D_{0}\right) \sim N(\mu, \Sigma)$, onde $D_{0}$ é o conjunto de informações no tempo $t=0$.

Queremos estimar o vetor de estado $\theta_{t}$ e o vetor de parâmetros $\varphi=\left(\gamma_{j k}, G_{t}, V_{t}, W_{t}\right)$.

\subsection{Estimação dos Parâmetros}

Seguindo o mesmo procedimento de estimação de máxima verossimilhança dos parâmetros dado na seção 4.3, isto é, a utilização do Algoritmo EM e do 
Filtro de Kalman, obtemos a seguinte solução das equações do passo M para uma particularização do modelo (6.1) supondo:

- uma única série temporal observada, isto é, $r=1$;

- o vetor de estado univariado, isto é, $\mathrm{p}=1$;

- $F_{t}=\sum_{j, k} \gamma_{j k} \psi_{j, k}(t / T)$;

- $G_{t}=G$;

- $V_{t}=V$;

- $W_{t}=W$.

i) Estimativas dos parâmetros $\gamma_{j k}$ :

Vamos substituir os índices $\mathrm{j}, \mathrm{k}$ de $\gamma_{j k}$ e $\psi_{j, k}(t / T)$ por um único índice $l$, onde $l=1, \ldots, s$ e $s$ é o número total de parâmetros $\gamma_{j k}$. Deste modo teremos que resolver $s$ equações do tipo:

$$
\sum_{t=1}^{n}\left[y_{t} x_{t}^{n} \psi_{l}(t / T)\right]-\sum_{t=1}^{n}\left[C_{t}\left(\sum_{i=1}^{s}\left(\hat{\gamma}_{i} \psi_{i}(t / T)\right) \psi_{l}(t / T)\right]=0, \quad l=1, \ldots, s,\right.
$$

onde

$$
\begin{aligned}
& x_{t}^{n}=E\left(\theta_{t} \mid y_{1}, \ldots, y_{n}\right) ; \\
& P_{t}^{n}=\operatorname{cov}\left(\theta_{t} \mid y_{1}, \ldots, y_{n}\right) ; \\
& C_{t}=P_{t}^{n}+x_{t}^{n} x_{t}^{n^{\prime}},
\end{aligned}
$$

Podemos montar um sistema para o conjunto das $s$ equações tal que $Z \hat{\gamma}=U$, onde

$$
\begin{gathered}
Z=\left(\begin{array}{cccc}
\sum_{t=1}^{n} C_{t} \psi_{1}^{2}(t / T) & \sum_{t=1}^{n} C_{t} \psi_{1}(t / T) \psi_{2}(t / T) & \ldots & \sum_{t=1}^{n} C_{t} \psi_{1}(t / T) \psi_{s}(t / T) \\
\sum_{t=1}^{n} C_{t} \psi_{1}(t / T) \psi_{2}(t / T) & \sum_{t=1}^{n} C_{t} \psi_{2}^{2}(t / T) & \ldots & \sum_{t=1}^{n} C_{t} \psi_{2}(t / T) \psi_{s}(t / T) \\
\vdots & \vdots & \ddots & \vdots \\
\sum_{t=1}^{n} C_{t} \psi_{1}(t / T) \psi_{s}(t / T) & \sum_{t=1}^{n} C_{t} \psi_{2}(t / T) \psi_{s}(t / T) & \ldots & \sum_{t=1}^{n} C_{t} \psi_{s}^{2}(t / T)
\end{array}\right) \\
\hat{\gamma}=\left(\begin{array}{c}
\hat{\gamma}_{1} \\
\hat{\gamma}_{2} \\
\vdots \\
\hat{\gamma}_{s}
\end{array}\right) \quad \mathrm{e} \\
U=\left(\begin{array}{c}
\sum_{t=1}^{n} y_{t} x_{t}^{n} \psi_{1}(t / T) \\
\sum_{t=1}^{n} y_{t} x_{t}^{n} \psi_{2}(t / T) \\
\vdots \\
\sum_{t=1}^{n} y_{t} x_{t}^{n} \psi_{s}(t / T)
\end{array}\right)
\end{gathered}
$$


E se existir a inversa de $Z$, temos $\hat{\gamma}=Z^{-1} U$.

ii) Estimativa do parâmetro $G$ :

$$
\hat{G}=\frac{\sum_{t=1}^{n} B_{t}}{\sum_{t=1}^{n} A_{t}},
$$

onde

$$
\begin{aligned}
& A_{t}=P_{t-1}^{n}+x_{t-1}^{n} x_{t-1}^{n^{\prime}}, \\
& B_{t}=P_{t, t-1}^{n}+x_{t}^{n} x_{t-1}^{n^{\prime}}, \\
& x_{t}^{n}, P_{t}^{n} \text { e } P_{t, t-1}^{n} \text { são obtidos pelo suavizador de Kalman. }
\end{aligned}
$$

iii) Estimativa do parâmetro W:

$$
\hat{W}=(1 / n) \sum_{t=1}^{n}\left[C_{t}-2 B_{t} \hat{G}_{t}+A_{t} \hat{G}_{t}^{2}\right] .
$$

iv) Estimativa do parâmetro V:

$$
\hat{V}=(1 / n) \sum_{t=1}^{n}\left[\left(y_{t}-\hat{F}_{t} x_{t}^{n}\right)^{2}+P_{t}^{n} \hat{F}_{t}^{2}\right],
$$

onde

$$
\hat{F}_{t}=\sum_{l=1}^{r} \hat{\gamma}_{l} \psi_{l}(t / T) .
$$

\subsection{Problemas de Estimação}

Ao implementarmos computacionalmente o procedimento de máxima verossimilhança e o algoritmo EM, não conseguimos estimar o parâmetro de perturbação do vetor de estado (matriz de covariâncias de $\theta_{t}, W$ ).

Observamos dos exemplos simulados que havia uma "compensação" entre as estimativas do parâmetro $F_{t}$ (obtido por máxima verossimilhança, algoritmo EM) e do vetor de estado $\theta_{t}$ (obtido pelo filtro de Kalman) provocada por diversos valores iniciais do parâmetro $W$ (covariância do vetor de estado $\theta_{t}$ ). 
Inicialmente julgamos tratar-se de um problema de identicabilidade como definido em Harvey (1989), mas não foi possível obter uma prova analítica para tal suposição.

Recorremos a métodos alternativos de obtenção de estimadores de máxima verossimilhança, utilizando uma "pseudo-verossimilhança" conhecida como verossimilhança perfilada.

A utilização de uma extensão da definição de verossimilhança (pseudo-verossimilhança) tem os seguintes objetivos:

i) reduzir a dimensão do vetor de parâmetro de perturbação;

ii) simplificar o cálculo através da pseudo-verossimilhança onde a construção da verossimilhança seria difícil;

iii) desenvolver métodos alternativos baseados em propriedades dos momentos, sem a necessidade da suposição de uma distribuição para os dados.

Considere $\phi$ o vetor de parâmetros. Adotamos uma partição $\phi^{\prime}=\left(\gamma^{\prime}, \lambda^{\prime}\right)$ de maneira que a função log-verossimilhança geral, $L(\gamma, \lambda)$, seja unicamente maximizada com relação a estes parâmetros dada a série $Y_{t}$. Seja $\hat{\lambda}_{\gamma}$ a estimativa de máxima verossimilhança dos parâmetros de perturbação quando $\gamma$ é fixado, supondo ainda que seja única. A função log-verossimilhança perfilada para $\gamma$ é definida por

$$
L^{*}(\gamma)=L\left(\gamma, \hat{\lambda}_{\gamma}\right)=\sup _{\lambda} L(\gamma, \lambda)
$$

A expressão (6.2) sugere um procedimento de maximização em duas etapas:

Etapa 1: consiste em achar um único $\hat{\lambda}_{\gamma}$ que maximiza $L(\gamma, \lambda)$ com relação a $\lambda$ supondo $\gamma$ constante;

Etapa 2: consiste em encontrar o valor de $\gamma$ que maximiza $L^{*}(\gamma)$.

Em geral, $\hat{\lambda}_{\gamma}$ diferirá de $\hat{\lambda}$ (estimativa usual de $\lambda$ ) por termos de ordem $O_{p}\left(n^{-1 / 2}\right)$. Os máximos de $L^{*}(\gamma)$ e $L(\gamma, \lambda)$ coincidem e, então, supondo que $\hat{\gamma}$ maximiza $(6.2)$, temos $L^{*}(\hat{\gamma}) \geq L^{*}(\gamma)$ ou $L\left(\hat{\gamma}, \hat{\lambda}_{\hat{\gamma}}\right) \geq L\left(\gamma, \hat{\lambda}_{\gamma}\right) \geq L(\gamma, \lambda)$.

Concluimos que as estimativas de máxima verossimilhança perfilada $\hat{\gamma}$ e $\hat{\lambda}_{\gamma}$ são iguais às estimativas de máxima verossimilhança usuais de $\gamma \mathrm{e} \lambda$, respectivamente.

Mais detalhes sobre as vantagens de tal método e comentários gerais ver Cordeiro (1992). 
O artigo de Richards (1961) apresenta o procedimento de máxima verossimilhança perfilada como um método de estimação de máxima verossimilhança quando o conhecimento de um ou mais parâmetros simplifica a função a ser maximizada. Ele desenvolve as estimativas da matriz de covariâncias assintóticas e aplica em exemplos de regressão.

Em Chan e Mak (1979) encontramos uma aplicação da verossimilhança perfilada em modelos com erros nas variáveis que apresenta problema de identificação nos parâmetros de variância.

No nosso problema, consideramos o parâmetro $W$ (matriz de covariâncias do vetor de estado $\theta_{t}$ ) como o parâmetro de perturbação a ser estimado na etapa 1 do procedimento de obtenção da função de máxima verossimilhança perfilada e os demais parâmetros serão estimados na etapa 2 .

\subsection{Exemplos de Séries Simuladas com Ondale- tas Haar em $F_{t}$}

Simulamos no programa S-plus dois exemplos de séries com mudanças de regime. Caracterizamos esta mudança considerando ondaletas Haar na matriz do sistema $F_{t}$. A Tabela 6.1 apresenta os exemplos e seus respectivos parâmetros simulados.

Implementamos na linguagem Fortran 90 um programa que calcula o filtro de Kalman no processo iterativo para estimar o estado e calcula as estimativas dos parâmetros utilizando o algoritmo EM em duas etapas: a primeira fixa o parâmetro $W$ e estima $F_{t}, G_{t}$ e $V$ e a segunda estima $W$ e fixa os demais parâmetros (verossimilhança perfilada).

Em um estudo anterior, observamos que as estimativas dos parâmetros $W$ obtidas na etapa 2 variavam segundo o valor inicial atribuído a $W$ na etapa 1 . Deste modo, estimamos modelos que supõem diversos valores iniciais de $W(0.001$, $0.01,0.1$ e 1 , respectivamente).

Os modelos estimados propostos neste capítulo supõem que a matriz do sistema $F_{t}$ pode captar a mudança de regime a partir da soma de ondaletas Haar, tal que

$$
F_{t}=\sum_{j, k} \gamma_{j k} \psi_{j, k}(t / T)
$$


Tabela 6.1: Parâmetros dos modelos simulados.

\begin{tabular}{c|c|c|c|c}
\hline \hline Modelo & $G_{t}$ & $F_{t}$ & $W$ & $V$ \\
\hline \hline Exemplo 1 & 1 & $\begin{array}{c}\text { Uma ondaleta Haar } \\
j=1, k=1\end{array}$ & 0.16 & 0.25 \\
\hline Exemplo 2 & 1 & $\begin{array}{c}\text { Três ondaletas Haar } \\
j=1,2, k=1, \ldots, 2^{j-1}\end{array}$ & 0.16 & 0.25 \\
\hline \hline
\end{tabular}

Consideramos variável a quantidade de parâmetros associados à ondaleta. Esta variação está associada ao total de dados $\left(n=256=2^{m}\right)$. O valor máximo de $m$ é a potência de dois do total de dados $n$. Ajustamos modelos para $m$ variando de 2 até 6 tal que

$$
j=1, \cdots, m \text { e } k=1, \cdots, 2^{j-1} \text {. }
$$

Observamos que o valor inicial para o parâmetro $F_{t}$ deve induzir a mudança de regime. Se isso não acontece, por exemplo, supor como valor inicial uma constante, esta variação pode se refletir no estado.

Os resultados obtidos são mostrados em uma tabela contendo o número de iteração total (depois de passar pelas duas etapas), o valor da verossimilhança $(-2 \log L)$ e as estimativas dos parâmetros $W, V$ e $G_{t}$ obtidas pelo Algoritmo EM. Apresentamos o resultado do teste BIC usado como critério para a escolha do melhor modelo (semelhante ao Capítulo 4). As estimativas do parâmetro $F_{t}$ e do estado são apresentadas graficamente, juntamente com o valor ajustado para $y_{t}$. 


\section{Exemplo 1:}

A Tabela 6.2 apresenta os resultados das estimativas para os modelos ajustados. Observamos que existe variação na estimativa de $W$ segundo o valor inicial considerado para o mesmo número de parâmetros de ondaletas utilizados (mesmo valor de $m$ ).

As Figuras 6.1 a 6.5 apresentam os gráficos da série, do estado e da matriz do sistema $F_{t}$ e suas respectivas estimativas para diversos valores iniciais de $W$ $(0.001,0.01,0.1$ e 1$)$. Cada figura especifica a ondaleta Haar com um número de coeficientes determinado $(m=2$ a $m=6)$.

Observamos das Figuras 6.1 a 6.5 conjuntamente com os resultados da Tabela 6.2 que a qualidade do ajuste para os diversos valores iniciais de $W$ se traduz na função BIC via $-2 \log L$. Observamos também que há uma grande variação nos valores estimados para $W$ e o melhor modelo nem sempre é o que apresenta a melhor estimativa de $W$.

A Figura 6.5 mostra as estimativas para diversos valores de $W$ e $m=6$. Da Tabela 6.2 observamos que as funções $-2 \log L$ diminuem à medida que incluímos mais coeficientes de ondaletas, isto é, quando aumentamos o valor de $m$, salvo para estes modelos, onde $-2 \log L$ é muito maior que para os modelos com $m=2$ a $m=5$, significando que não precisamos de tantas ondaletas (63 no total) para estimar $F_{t}$.

Segundo o critério BIC, o melhor modelo (comparando todos os valores de $m$ ) é o que utiliza a ondaleta Haar com $m=4$ e o valor inicial para $W$ igual a 0.01 . 
Tabela 6.2: Resultados obtidos para o exemplo 1 utilizando os modelos com ondaletas Haar, $m=2, \ldots, 6$, respectivamente, para diversos valores iniciais de $W .\left({ }^{*}\right)$ Melhor modelo segundo o BIC.

\begin{tabular}{|c|c|c|c|c|c|c|}
\hline$W$ inicial & $\begin{array}{c}\text { Iteração } \\
\text { Total }\end{array}$ & $-2 \log L$ & W & V & $G_{t}$ & $\mathrm{BIC}$ \\
\hline \multicolumn{7}{|c|}{ Haar, $m=2$} \\
\hline 0.001 & 11 & 170.6974 & 0.0025 & 0.4187 & 0.9809 & 182.7387 \\
\hline 0.01 & 11 & 168.4576 & 0.0249 & 0.4188 & 0.9809 & 180.4989 \\
\hline 0.1 & 11 & 166.9631 & 0.1224 & 0.4077 & 0.9781 & 179.0044 \\
\hline 1 & 11 & 166.1723 & 0.5851 & 0.3979 & 0.9756 & $178.2136\left(^{*}\right)$ \\
\hline \multicolumn{7}{|c|}{ Haar, $\mathrm{m}=3$} \\
\hline 0.001 & 11 & 172.6397 & 0.0029 & 0.4462 & 0.9877 & 194.3139 \\
\hline 0.01 & 11 & 159.6438 & 0.0247 & 0.3916 & 0.9803 & 181.3180 \\
\hline 0.1 & 11 & 157.6788 & 0.1835 & 0.3789 & 0.9779 & 179.3530 \\
\hline 1 & 11 & 154.6053 & 0.7190 & 0.2750 & 0.9617 & $176.2795\left(^{*}\right)$ \\
\hline \multicolumn{7}{|c|}{ Haar, $m=4$} \\
\hline 0.001 & 11 & 155.4737 & 0.0002 & 0.6581 & 0.9917 & 196.4138 \\
\hline 0.01 & 11 & 131.1500 & 0.0208 & 0.2499 & 0.9657 & $172.0901\left(^{*}\right)$ \\
\hline 0.1 & 11 & 134.7714 & 0.1885 & 0.2341 & 0.9627 & 175.7115 \\
\hline 1 & 11 & 134.0386 & 0.8335 & 0.2202 & 0.9568 & 174.9788 \\
\hline \multicolumn{7}{|c|}{ Haar, $m=5$} \\
\hline 0.001 & 11 & 118.5158 & 0.00004 & 0.5126 & 0.9905 & 197.9877 \\
\hline 0.01 & 11 & 119.5431 & 0.0183 & 0.4483 & 0.9850 & 199.0151 \\
\hline 0.1 & 11 & 103.0810 & 0.1820 & 0.2187 & 0.9646 & 182.5530 \\
\hline 1 & 11 & 102.6562 & 0.8424 & 0.2023 & 0.9589 & $182.1282\left(^{*}\right)$ \\
\hline \multicolumn{7}{|c|}{ Haar, $m=6$} \\
\hline 0.001 & 6 & 226.2889 & 0.0031 & "0.2353 & (20.9662 & $382.8246\left(^{*}\right)$ \\
\hline 0.01 & 6 & 232.8214 & 0.0189 & 0.2661 & 0.9678 & 389.3770 \\
\hline 0.1 & 6 & 290.1902 & 0.1785 & 0.3422 & 0.9561 & 446.7258 \\
\hline 1 & 6 & 290.4251 & 1.8799 & 0.2594 & 0.9235 & 446.9607 \\
\hline
\end{tabular}



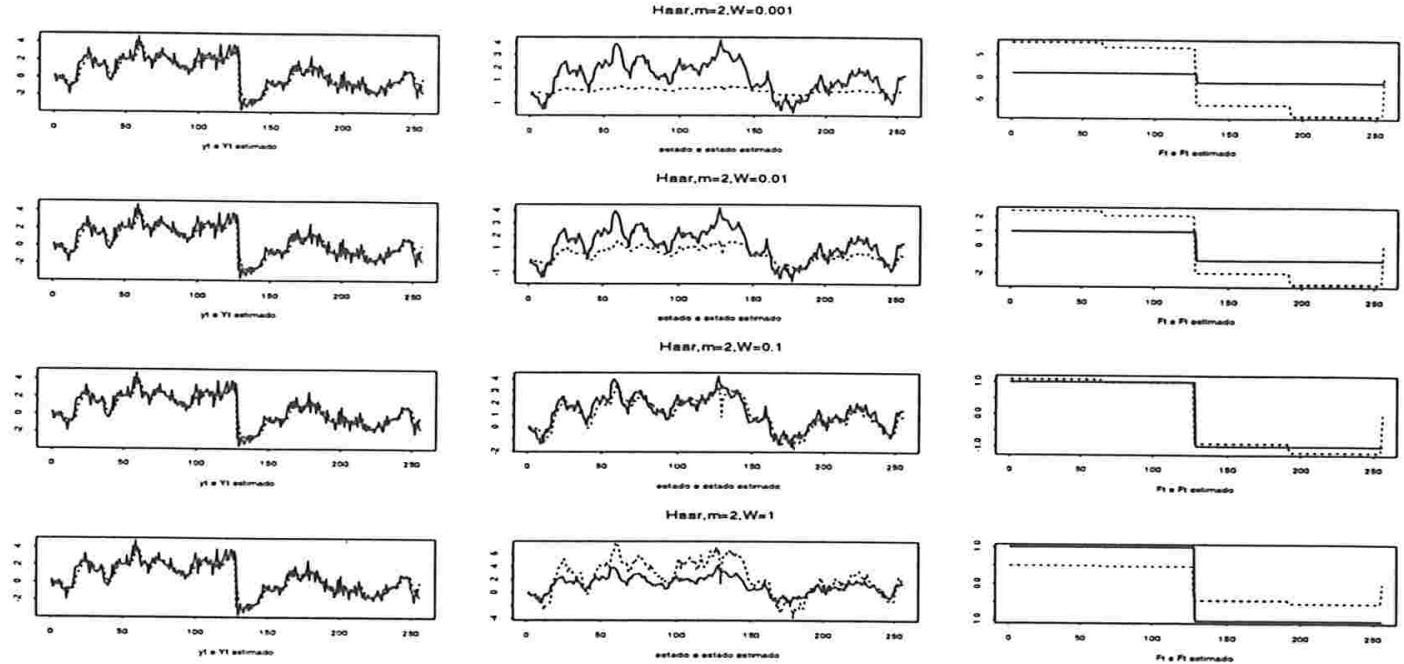

Figura 6.1: Gráficos da série $y_{t}$, estado $\theta_{t}$ e matriz do sistema $F_{t}$ (linha cheia) e suas respectivas estimativas (tracejado) para os modelos utilizando a ondaleta Haar com $\mathrm{m}=2$ para diversos $W$ iniciais (Exemplo 1 ).
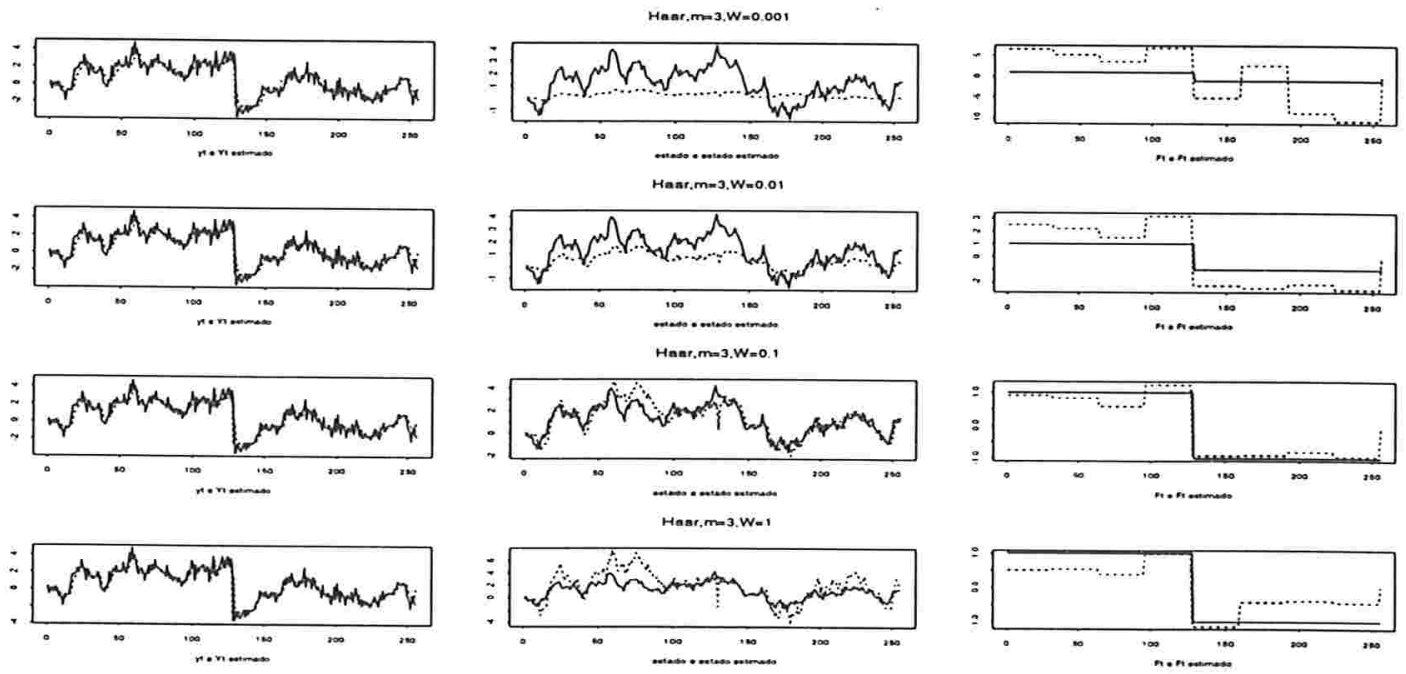

Figura 6.2: Gráficos da série $y_{t}$, estado $\theta_{t}$ e matriz do sistema $F_{t}$ (linha cheia) e suas respectivas estimativas (tracejado) para os modelos utilizando a ondaleta Haar com $\mathrm{m}=3$ para diversos $W$ iniciais (Exemplo 1 ). 

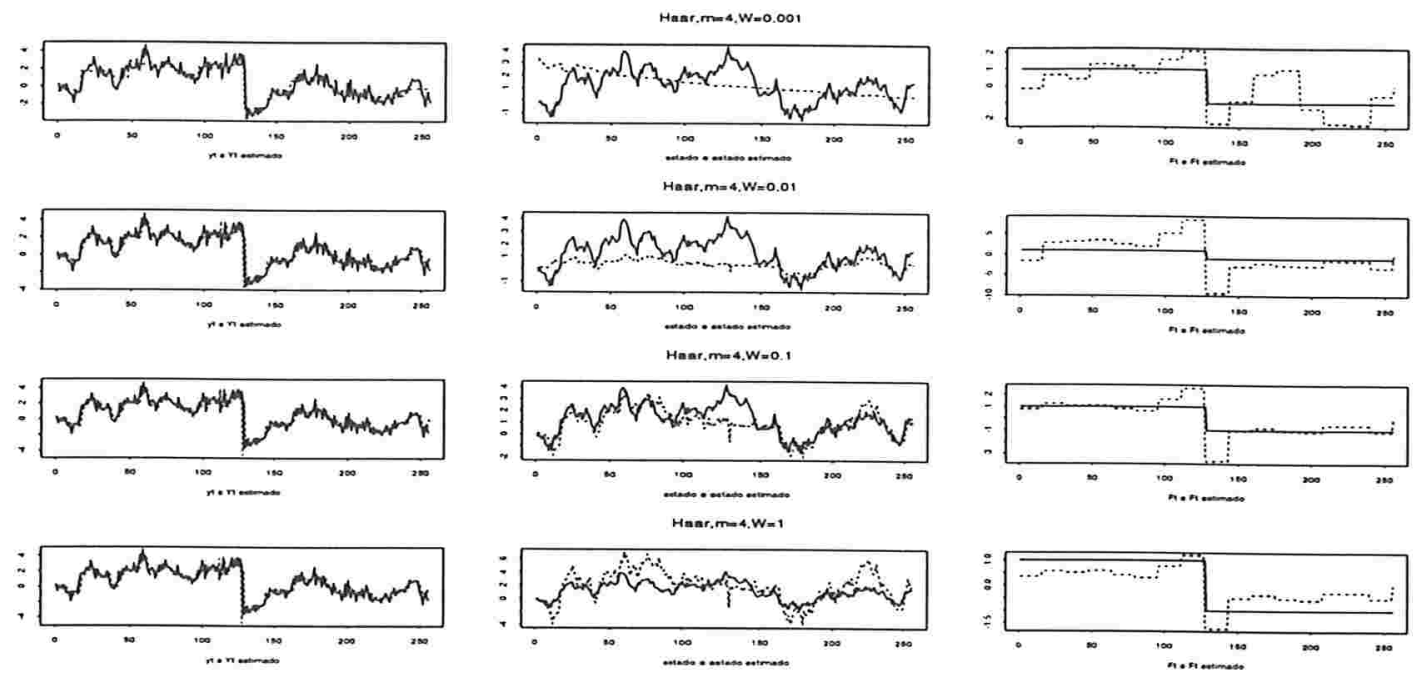

Figura 6.3: Gráficos da série $y_{t}$, estado $\theta_{t}$ e matriz do sistema $F_{t}$ (linha cheia) e suas respectivas estimativas (tracejado) para os modelos utilizando a ondaleta Haar com $\mathrm{m}=4$ para diversos $W$ iniciais (Exemplo 1 ).
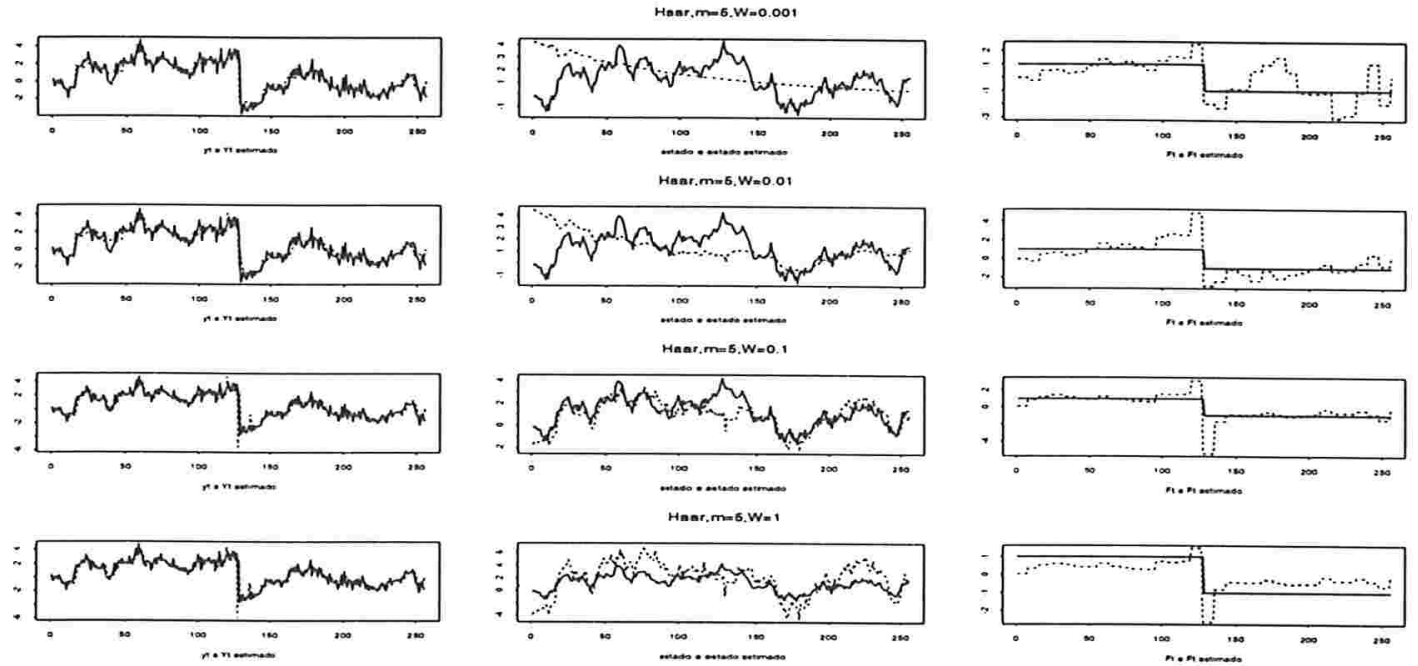

Figura 6.4: Gráficos da série $y_{t}$, estado $\theta_{t}$ e matriz do sistema $F_{t}$ (linha cheia) e suas respectivas estimativas (tracejado) para os modelos utilizando a ondaleta Haar com $\mathrm{m}=5$ para diversos $W$ iniciais (Exemplo 1 ). 

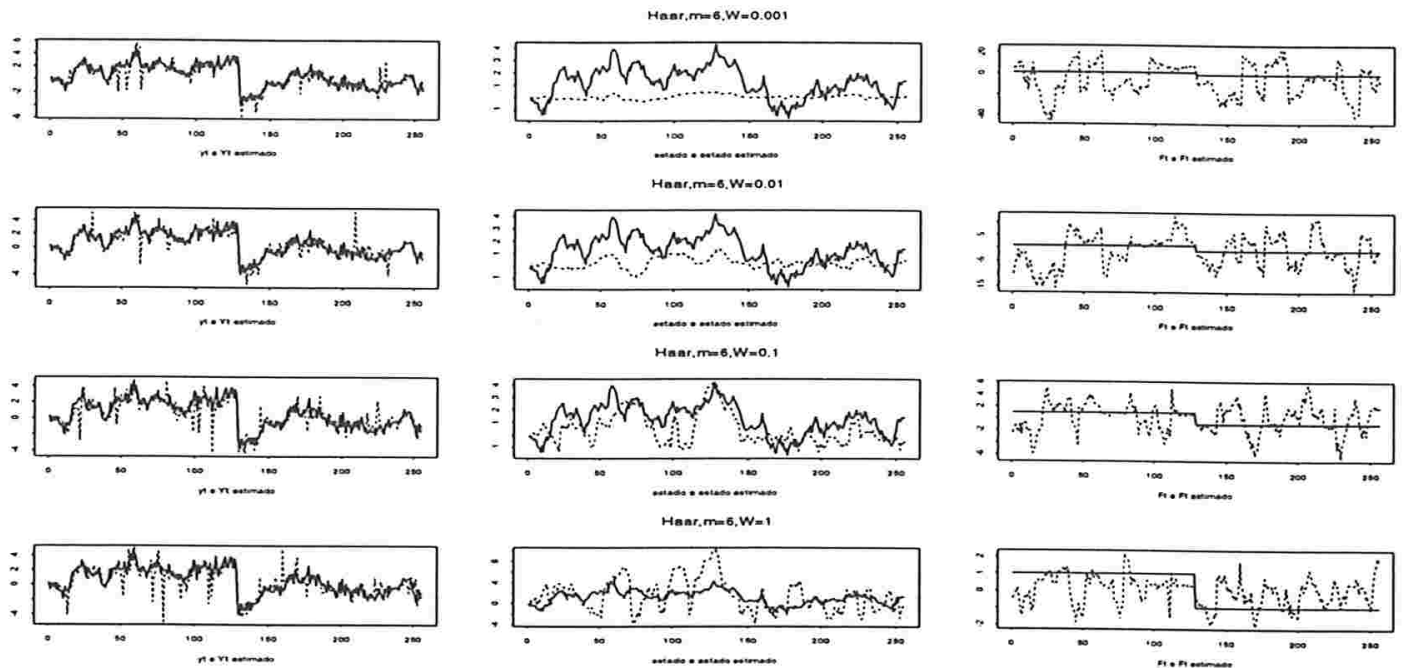

Figura 6.5: Gráficos da série $y_{t}$, estado $\theta_{t}$ e matriz do sistema $F_{t}$ (linha cheia) e suas respectivas estimativas (tracejado) para os modelos utilizando a ondaleta Haar com $m=6$ para diversos $W$ iniciais (Exemplo 1 ).

Exemplo 2:

Da Tabela 6.3 observamos que a função $-2 \log L$ varia muito (para um mesmo valor de $m$ ) segundo o valor inicial do parâmetro $W$. Observamos que para $m=6$ os modelos estimados não apresentaram bons resultados (ver Figura 6.10). E para os demais, os melhores oscilaram entre $W$ inicial igual a 0.1 (para $m=2$ e $m=3$ ) e $W$ inicial igual a 0.01 (para $m=4$ e $m=5$ ).

As Figuras 6.6 a 6.10 apresentam as estimativas da série, do estado e da matriz do sistema $F_{t}$ para os diversos valores iniciais de $W$ e diversos números de parâmetros de ondaletas $(m=2$ a $m=6)$. Observamos que os gráficos que apresentam melhor ajuste em cada grupo (fixar $m$, o número de ondaletas) são os que apresentam menor $-2 \log L$, e conseqüentemente menor BIC.

O melhor modelo, segundo o critério BIC, foi o que utilizou 0.1 como valor inicial para $W$ e apenas três (3) ondaletas Haar para estimar $F_{t}$ (isto é, $m=2$ ). 
Tabela 6.3: Resultados obtidos para o exemplo 2 utilizando os modelos com ondaletas Haar, $m=2, \ldots, 6$, respectivamente, para diversos valores iniciais de $W .\left({ }^{*}\right)$ Melhor modelo segundo o BIC.

\begin{tabular}{c|c|c|c|c|c|c}
\hline \hline inicial & $\begin{array}{c}\text { Iteração } \\
\text { Total }\end{array}$ & $-2 \operatorname{logL}$ & $\mathrm{W}$ & $\mathrm{V}$ & $G_{t}$ & BIC \\
\hline \hline \multicolumn{7}{c}{ Haar, $\mathrm{m}=2$} \\
\hline \hline 0.001 & 11 & 276.3841 & 0.1145 & 0.5673 & 0.9974 & 288.4254 \\
0.01 & 11 & 275.9883 & 0.1645 & 0.5589 & 0.9972 & 288.0296 \\
0.1 & 11 & 275.8095 & 0.1976 & 0.5563 & 0.9971 & $287.8507\left(^{*}\right)$ \\
1 & 11 & 439.0225 & 24.6767 & 0.8286 & 0.9896 & 451.0637 \\
\hline \hline \multicolumn{7}{c}{ Haar, $\mathrm{m}=3$} \\
\hline \hline 0.001 & 11 & 347.4153 & 0.2321 & 0.6532 & 0.9932 & 369.0895 \\
0.01 & 11 & 274.9865 & 0.0607 & 0.5595 & 0.9976 & 296.6607 \\
0.1 & 11 & 274.8048 & 0.0757 & 0.5520 & 0.9974 & $296.4790\left(^{*}\right)$ \\
1 & 11 & 347.1638 & 0.3559 & 0.6457 & 0.9928 & 368.8380 \\
\hline \hline \multicolumn{7}{|c}{ Haar, $\mathrm{m}=4$} \\
\hline \hline 0.001 & 11 & 278.3452 & 0.0115 & 0.6354 & 0.9984 & 319.2853 \\
0.01 & 11 & 277.0348 & 0.1018 & 0.6736 & 0.9984 & $317.9749\left(^{*}\right)$ \\
0.1 & 11 & 414.0240 & 0.3379 & 0.6289 & 0.9939 & 454.9641 \\
1 & 11 & 487.6912 & 2.3368 & 0.7554 & 0.9829 & 528.6313 \\
\hline \hline \multicolumn{7}{c}{ Haar, $\mathrm{m}=5$} \\
\hline \hline 0.001 & 6 & 265.4127 & 0.0065 & 0.6289 & 0.9987 & 344.8847 \\
0.01 & 6 & 264.8161 & 0.0360 & 0.6287 & 0.9987 & $344.2881\left(^{*}\right)$ \\
0.1 & 6 & 384.2977 & 0.1975 & 0.5958 & 0.9964 & 463.7696 \\
1 & 6 & 454.4179 & 1.5424 & 0.7032 & 0.9857 & 533.8899 \\
\hline \hline \multicolumn{7}{c}{ Haar, $\mathrm{m}=6$} \\
\hline 0.001 & 6 & 917.9634 & 0.0006 & 9.4810 & 0.9543 & 1074.4990 \\
0.01 & 6 & 771.2515 & 0.0318 & 2.9074 & 0.9825 & 927.7872 \\
0.1 & 6 & 766.0028 & 0.3089 & 2.0470 & 0.9465 & 922.5385 \\
1 & 6 & 740.2658 & 3.2247 & 2.3098 & 0.9616 & $896.8014\left(^{*}\right)$ \\
\hline \hline
\end{tabular}



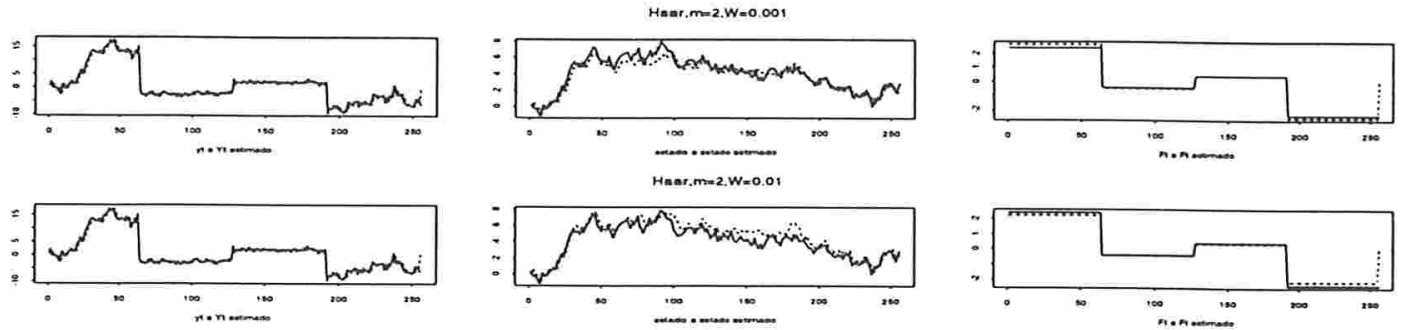

Haar,m=2.W-0.01
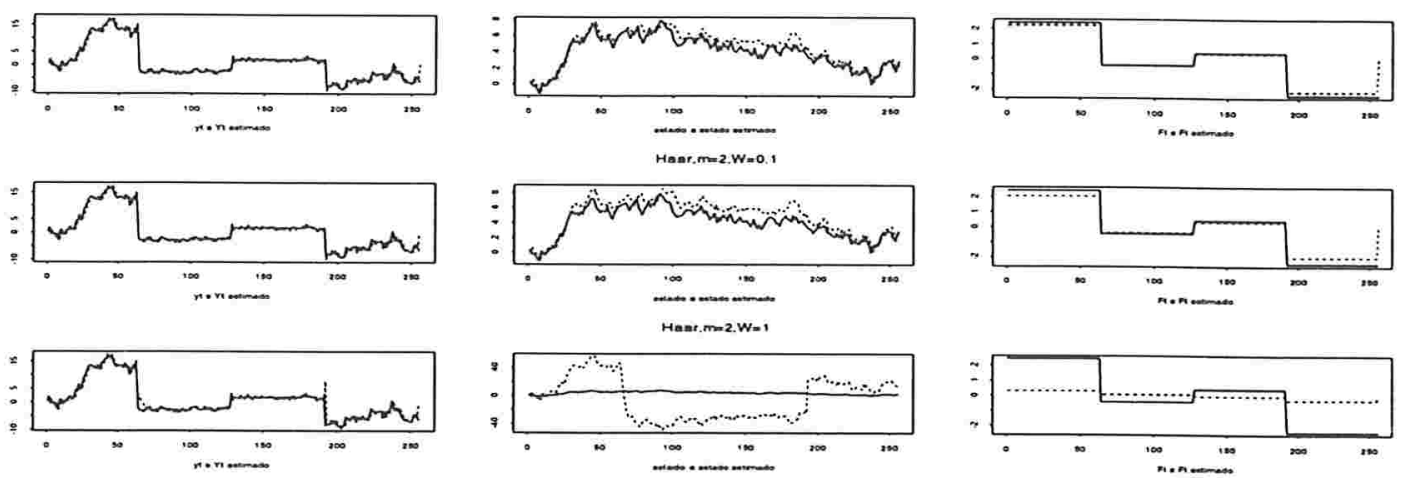

Figura 6.6: Gráficos da série $y_{t}$, estado $\theta_{t}$ e matriz do sistema $F_{t}$ (linha cheia) e suas respectivas estimativas (tracejado) para os modelos utilizando a ondaleta Haar com $\mathrm{m}=2$ para diversos $W$ iniciais (Exemplo 2).
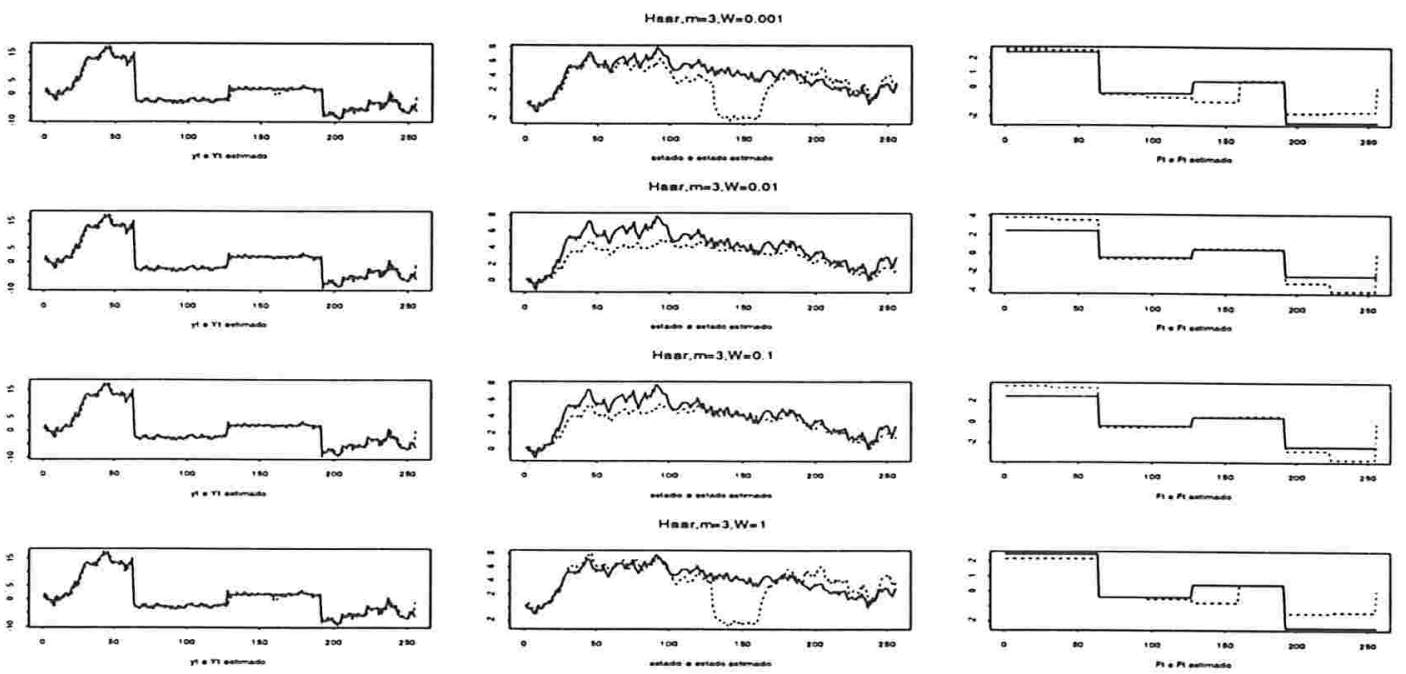

Figura 6.7: Gráficos da série $y_{t}$, estado $\theta_{t}$ e matriz do sistema $F_{t}$ (linha cheia) e suas respectivas estimativas (tracejado) para os modelos utilizando a ondaleta Haar com $\mathrm{m}=3$ para diversos $W$ iniciais (Exemplo 2). 

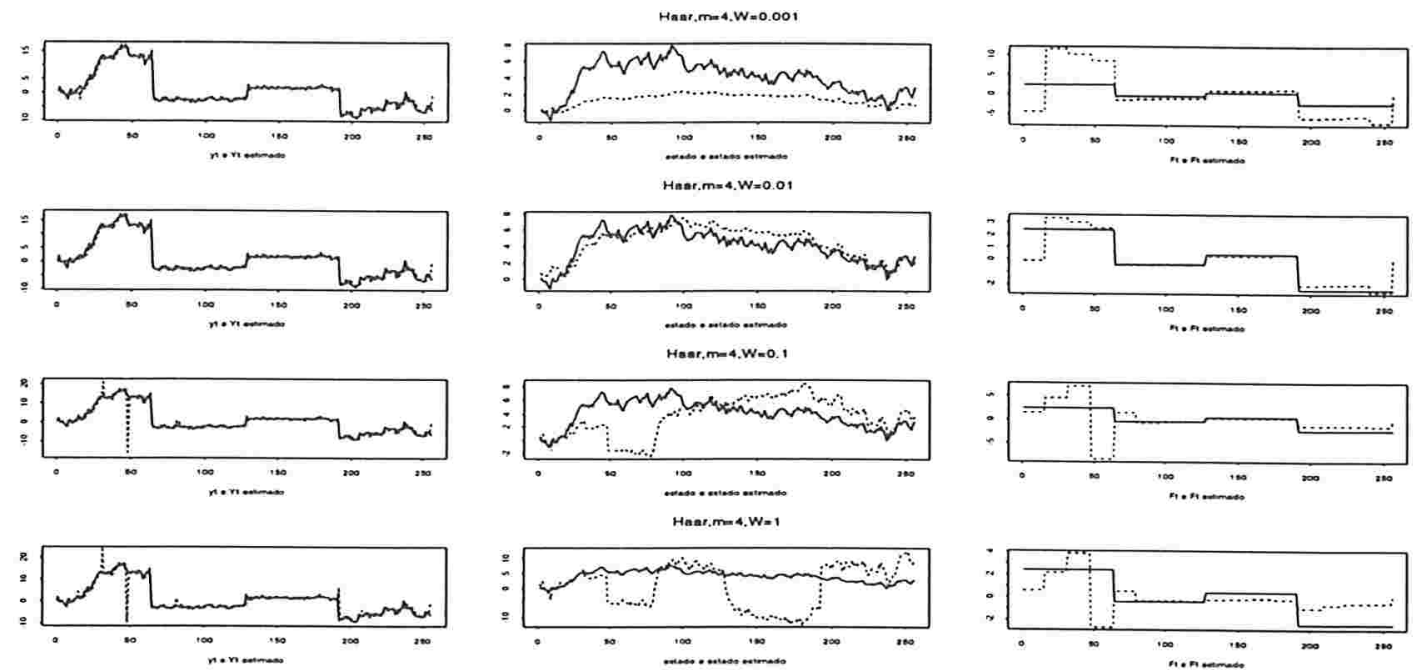

Figura 6.8: Gráficos da série $y_{t}$, estado $\theta_{t}$ e matriz do sistema $F_{t}$ (linha cheia) e suas respectivas estimativas (tracejado) para os modelos utilizando a ondaleta Haar com $\mathrm{m}=4$ para diversos $W$ iniciais (Exemplo 2).
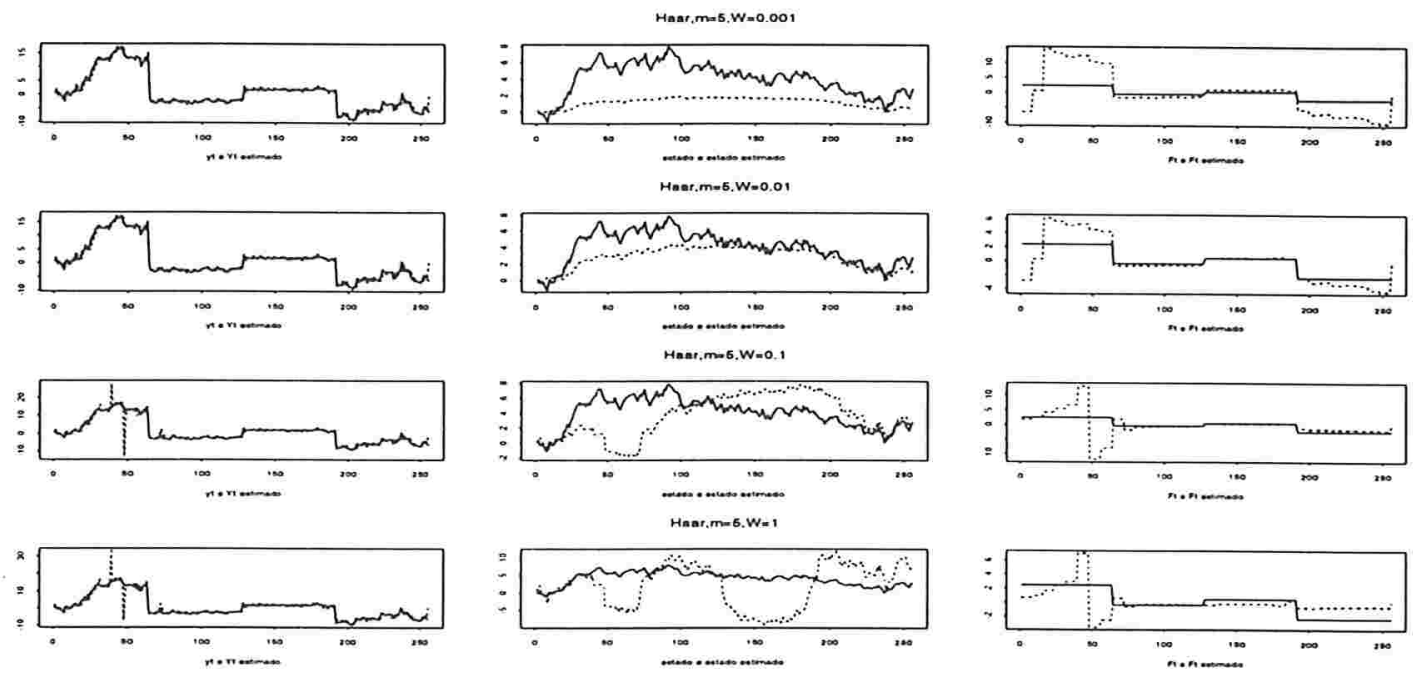

Figura 6.9: Gráficos da série $y_{t}$, estado $\theta_{t}$ e matriz do sistema $F_{t}$ (linha cheia) e suas respectivas estimativas (tracejado) para os modelos utilizando a ondaleta Haar com $\mathrm{m}=5$ para diversos $W$ iniciais (Exemplo 2). 

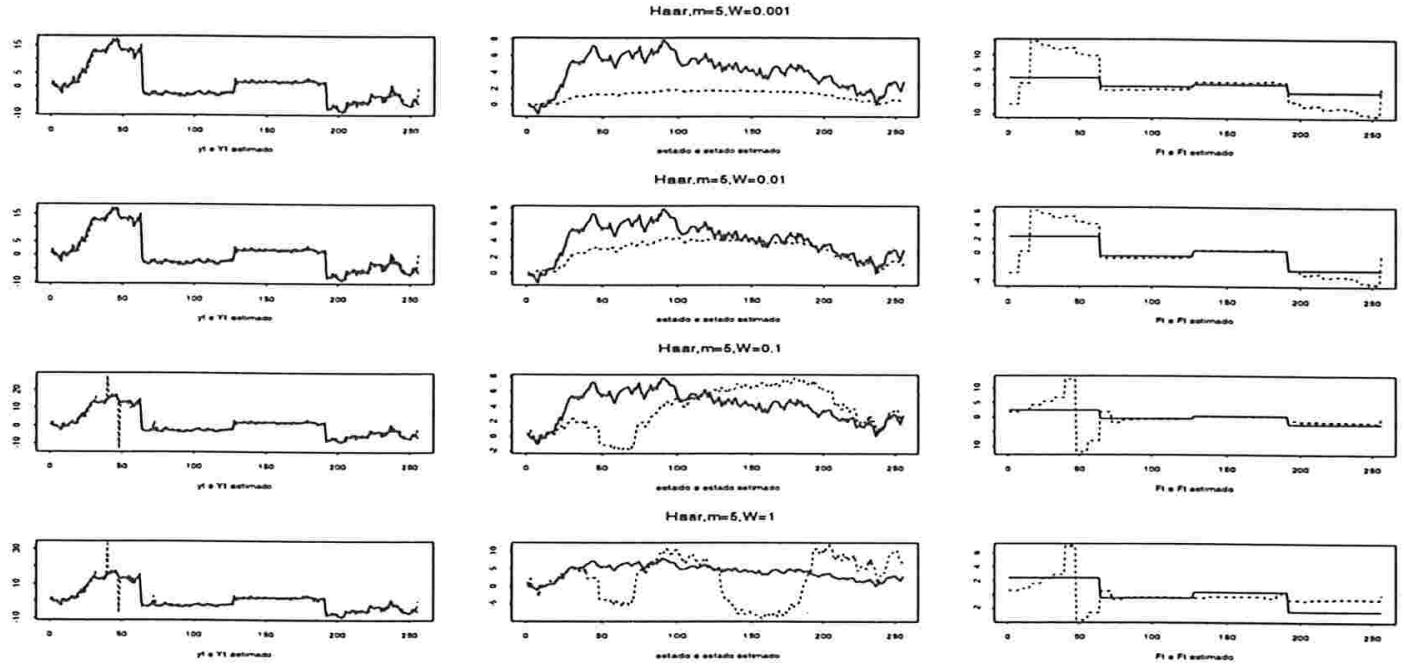

Figura 6.10: Gráficos da série $y_{t}$, estado $\theta_{t}$ e matriz do sistema $F_{t}$ (linha cheia) e suas respectivas estimativas (tracejado) para os modelos utilizando a ondaleta Haar com $\mathrm{m}=6$ para diversos $W$ iniciais (Exemplo 2). 


\section{Capítulo 7}

\section{Propriedades dos Estimadores e Convergência do Algoritmo EM}

\subsection{Introdução}

Problemas de inferência em modelos de espaço de estados são abordados a partir da existência de uma teoria assintótica. Dentro de condições apropriadas, tanto os parâmetros obtidos por máxima verossimilhança quanto o estado estimado via filtro de Kalman, são consistentes e assintoticamente normais. Os artigos Ljung e Caines (1979) e Spall e Wall (1984) abordam a teoria assintótica para estes modelos.

Todos os estimadores (Capítulos 4, 5 e 6 ) foram obtidos pelo algoritmo EM e são estimadores de máxima verossimilhança. Segundo Wu (1983), o algoritmo EM apresenta inúmeras vantagens do ponto de vista computacional, como fácil implementação para os passos E e M, soluções fechadas para o passo $\mathrm{M}$ em diversos casos, utilização de programas estatísticos para obtenção de resultados, o que diminui o tempo de programação e a não necessidade de grande espaço para armazenamento, podendo ser processado em computadores pequenos. Na seção 7.2 discutiremos propriedades de convergência do algoritmo EM.

Como já comentado no Capítulo 2, o algoritmo EM não fornece o erro padrão dos estimadores da forma convencional (não calcula a matriz de segundas derivadas parciais). Deste modo, existem métodos alternativos para a obtenção de tais erros. Na seção 7.3 apresentaremos alguns destes métodos. 
E finalmente na seção 7.4 introduzimos a técnica computacionalmente intensiva, bootstrap, aplicada a modelos de espaço de estados que também fornecerá uma caracterização para os estimadores.

\subsection{Convergência do Algoritmo EM}

O artigo Wu (1983) aborda dois aspectos da convergência do algoritmo EM: i) se o algoritmo EM encontra um máximo local ou um valor estacionário da função de verossimilhança;

ii) se o algoritmo EM converge.

Estas questões foram respondidas analisando-se o algoritmo EM como um algoritmo especial de otimização e utilizando-se resultados existentes na literatura de otimização.

Destacamos os resultados de convergência aplicados a duas situações práticas. Mais detalhes ver $\mathrm{Wu}$ (1983).

i) Se os dados podem ser descritos como pertencentes à família exponencial (nosso caso) com espaço paramétrico compacto, então todos os limites de qualquer seqüência do algoritmo EM são pontos estacionários da função de verossimilhança; ii) se a função de verossimilhança for unimodal e certas condições de diferenciabilidade forem satisfeitas, então o algoritmo EM converge para a única estimativa de máxima verossimilhança.

Mais detalhes sobre convergência do algoritmo EM podem ser encontrados em Dempster et al. (1977), Boyles (1983) e Wu (1983).

\subsection{Erros Padrões dos Estimadores}

Como já vimos, o algoritmo EM fornece um estimador de máxima verossimilhança para os parâmetros. Apesar de todas as vantagens da sua utilização, o algoritmo EM não fornece um estimador consistente para os erros padrões das estimativas de máxima verossimilhança obtidas. A seguir apresentamos alternativas para o cálculo da matriz de segundas derivadas. 
Na seção 4.4 de Tanner (1996), encontramos uma ampla discussão sobre o cálculo dos erros padrões dos estimadores. Os métodos apresentados são o da computação direta (Meilijson, 1989), o método de Louis (Louis, 1982), método de simulação e uso de iteradas EM. A abordagem utilizada é a da Estatística Bayesiana, onde o algoritmo EM fornece a moda da distribuição a posteriori $p(\varphi \mid Y)$, com $\varphi$ o vetor de parâmetros a serem estimados e $Y$ a série observada. Neste contexto, o objetivo é a obtenção da matriz hessiana de $\log p(\varphi \mid Y)$, dada por

$$
\left.\frac{-\partial^{2} \log p(\varphi \mid Y)}{\partial \varphi^{2}}\right|_{\varphi^{*}},
$$

onde $\varphi^{*}$ é a moda da distribuição a posteriori.

Meilijson (1989) sugere uma alternativa numérica que consiste em perturbar $\varphi^{*}=\left(\varphi_{1}^{*}, \ldots, \varphi_{d}^{*}\right)$, onde $d$ é o total de parâmetros, adicionando um valor $\varepsilon>0$ em cada coordenada e avaliar $\partial \log p(\varphi \mid Y) / \partial \varphi$ no parâmetro perturbado $\tilde{\varphi}$. Assim, a i-ésima linha da matriz hessiana é aproximadamente

$$
\frac{1}{\varepsilon}\left[\left.\frac{\partial \log p(\varphi \mid Y)}{\partial \varphi}\right|_{\tilde{\varphi}}-\left.\frac{\partial \log p(\varphi \mid Y)}{\partial \varphi}\right|_{\varphi^{*}}\right]
$$

O método de Louis (1982) baseia a obtenção da matriz de informação no Princípio da Falta de Informação que consiste em considerar

$$
\text { informação observada = informação completa - informação faltando. }
$$

Podemos traduzir este princípio utilizando as funções de probabilidade condicionais

$$
\log p(\varphi \mid Y)=\log p(\varphi \mid Y, \theta)-\log p(\theta \mid Y, \varphi) .
$$

Se integrarmos ambos os lados em relação a $p(\theta \mid Y, \varphi)$ e tomarmos as derivadas segundas em relação a $\varphi$, obtemos

$$
\begin{aligned}
\frac{-\partial^{2} \log p(\varphi \mid Y)}{\partial \varphi^{2}}= & -\int_{\theta} \frac{\partial^{2} \log p(\varphi \mid Y, \theta)}{\partial \varphi^{2}} p(\theta \mid Y, \varphi) d \theta \\
& +\int_{\theta} \frac{\partial^{2} \log p(\theta \mid Y, \varphi)}{\partial \varphi^{2}} p(\theta \mid Y, \varphi) d \theta .
\end{aligned}
$$


O resultado principal de Louis (1982) é dado a seguir. Para mais detalhes e demonstração ver o artigo.

$$
-\frac{\partial^{2} \int_{\theta} \log p(\theta \mid Y, \varphi) p(\theta \mid Y, \varphi) d \theta}{\partial \varphi^{2}}=\operatorname{var}\left[\frac{\partial \log p(\varphi \mid Y, \theta)}{\partial \theta}\right] .
$$

Aplicando (7.2) em (7.1) obtém-se uma alternativa para o cálculo da matriz de informação. Para os casos com dificuldades de uma forma analítica para a matriz de informação calculada dessa forma, Tanner (1996) sugere métodos de simulação.

\subsection{Método Bootstrap}

\subsubsection{Introdução}

O método bootstrap é uma técnica computacionalmente intensiva com recente utilização em estatística. Foi introduzido na literatura por Efron (1979) e permite uma avaliação da variabilidade da estimativa usando somente os dados. A idéia por trás do método é re-amostrar as observações originais de modo adequado com o objetivo de se construir um conjunto de "pseudo-dados". Para este novo conjunto obtém-se novas estimativas. E podemos calcular medidas de variabilidade, intervalos de confiança e estimativa de viés.

A amostragem é feita com reposição e dependendo da informação da distribuição dos dados, o bootstrap é classificado como paramétrico (distribuição conhecida) ou não-paramétrico (distribuição desconhecida). No primeiro caso, amostramos a distribuição e no segundo tiramos uma amostra aleatória dos dados originais.

Em séries temporais no domínio do tempo, existem duas abordagens para a realização da re-amostragem, considerando-se que os dados não são independentes ao longo do tempo. A primeira e mais simples é análoga ao modelo re-amostrado em regressão e consta de três (3) etapas básicas

i) ajustar um modelo aos dados;

ii) construir os resíduos do modelo ajustado;

iii) gerar novas séries incorporando amostras aleatórias dos resíduos no modelo ajustado. 
A segunda abordagem propõe não re-amostrar os resíduos, mas sim blocos de observações consecutivas. No caso de séries com mudanças de regimes, este método pode perder informações importantes sobre o momento da mudança.

Para um aprofundamento da aplicação de bootstrap em séries temporais, sugerimos Efron e Tibshirani (1993), capítulo 8 e Davison e Hinkley (1997), capítulo 8.

O recente trabalho de Franco (1998) aborda a utilização do bootstrap (paramétrico e não-paramétrico) para estudar o comportamento dos hiperparâmetros em modelos de espaço de estados nos casos de nível e tendência linear locais. Também são calculados intervalos de confiança e feitas comparações quanto à probabilidade de cobertura.

O método computacional bootstrap será utilizado neste trabalho com o objetivo de obtermos os erros padrões dos estimadores.

Em Franco (1998) encontramos um estudo de simulação de modelos em espaço de estados com o objetivo de comparar o comportamento das estimativas bootstrap com os resultados assintóticos padrões. Os resultados se mostraram satisfatórios no sentido que os erros padrões obtidos pelo método bootstrap são mais acurados.

\subsubsection{Algoritmo Bootstrap}

Neste trabalho, o bootstrap será utilizado como um método de obtenção de precisão das estimativas de máxima verossimilhança dos parâmetros do modelo linear em espaço de estado. As idéias discutidas aqui podem ser encontradas com mais detalhes em Stoffer e Wall (1991).

Suponha o modelo na forma de espaço de estados já discutido no capítulo 2

$$
\begin{aligned}
& y_{t}=F_{t} \theta_{t}+v_{t}, \\
& \theta_{t}=G_{t} \theta_{t-1}+w_{t} .
\end{aligned}
$$

Definimos como em Anderson e Moore (1979) as inovações $\epsilon_{t}$, a matriz de covariâncias das inovações $\Sigma_{t}$ e o ganho de Kalman, $K_{t}$

$$
\begin{aligned}
\epsilon_{t} & =y_{t}-F_{t} x_{t}^{t-1}, \\
\Sigma_{t} & =F_{t} P_{t}^{t-1} F_{t}^{\prime}+V_{t}, \\
K_{t} & =P_{t}^{t-1} F_{t}^{\prime}\left(\Sigma_{t}\right)^{-1},
\end{aligned}
$$


onde $x_{t}^{t-1}$ e $P_{t}^{t-1}$ são obtidos do filtro de Kalman.

Podemos representar o sistema (7.3) na forma das inovações (ver Anderson e Moore (1979), página 231) e obtemos

$$
\begin{aligned}
y_{t} & =F_{t} x_{t}^{t}+\epsilon_{t}, \\
x_{t}^{t} & =G_{t} x_{t}^{t-1}+G_{t} K_{t} \epsilon_{t} .
\end{aligned}
$$

O procedimento do método de simulação Monte Carlo bootstrap para modelos na forma de espaço de estados é definido por um algoritmo de quatro (4) passos. Considere que as estimativas de $\varphi, \hat{\varphi}=(\hat{F}, \hat{G}, \hat{V}, \hat{W})$ e o filtro de Kalman tenham sido obtidos. Conseqüentemente, temos as inovações estimadas, $\epsilon_{t}$, avaliadas em $\hat{\varphi}, \epsilon_{t}(\hat{\varphi})$.

PASSO 1: Construa as inovações padronizadas,

$$
e_{t}(\hat{\varphi})=\Sigma_{t}^{-1 / 2}(\hat{\varphi}) \epsilon_{t}(\hat{\varphi})
$$

onde $\Sigma_{t}^{-1 / 2}(\hat{\varphi})$ é a inversa da matriz raiz quadrada de $\Sigma_{t}(\hat{\varphi})$. Usando (7.8) garantimos que todos os resíduos têm, pelo menos, os dois primeiros momentos.

PASSO 2: Retire uma amostra de tamanho $T$, com reposição, de $\left\{e_{t}(\hat{\varphi}), 1 \leq t \leq\right.$ $T\}$ e obtenha $\left\{e_{t}^{*}(\hat{\varphi}), \quad 1 \leq t \leq T\right\}$ que é uma amostra bootstrap das inovações padronizadas.

PASSO 3: Construa os dados bootstrap $\left\{y_{t}^{*}, \quad 1 \leq t \leq T\right\}$ a partir da representação das observações sob forma das inovações, (7.7), usando $\left\{e_{t}^{*}(\hat{\varphi}), \quad 1 \leq t \leq T\right\}$ no lugar de $\left\{e_{t}(\hat{\varphi}), \quad 1 \leq t \leq T\right\}$. Obtemos

$$
\begin{aligned}
y_{t}^{*} & =F_{t} x_{t}^{t}+\Sigma_{t}^{1 / 2}(\hat{\varphi}) e_{t}^{*}(\hat{\varphi}), \\
x_{t}^{t} & =G_{t} x_{t}^{t-1}+G_{t} K_{t} \Sigma_{t}^{1 / 2}(\hat{\varphi}) e_{t}^{*}(\hat{\varphi}),
\end{aligned}
$$

onde os valores obtidos pelo Filtro de Kalman, bem como os parâmetros $\hat{\varphi}$ são mantidos fixos.

Com os novos dados bootstrap, calcular novamente o Filtro de Kalman e o algoritmo EM e obter novas estimativas $\hat{\varphi}^{*}$.

PASSO 4: Repetir os passos (2) e (3) um número $N$ de vezes e obter repetições para o conjunto $\left\{\hat{\varphi}^{* i}, 1 \leq i \leq N\right\}$. Estimar a distribuição de $\hat{\varphi}$ a partir de $\hat{\varphi}^{* i}$.

Uma propriedade importante deste método é que o bootstrap aplicado às inovações produzirá erros padrões assintoticamente consistentes. No apêndice de Stoffer e Wall (1991) encontramos a justificativa assintótica do método bootstrap. 


\section{Capítulo 8}

\section{Aplicação em Dados Reais e Conclusão}

\subsection{Introdução}

Este último capítulo apresenta uma aplicação a uma série real dos modelos desenvolvidos neste trabalho, com a implementação computacional do método bootstrap para obtenção do erros padrões dos estimadores.

E finalmente são apontadas conclusões e sugestões para trabalhos posteriores.

\subsection{Aplicação: Spread Diário entre dois Títulos da Dívida Externa Brasileira}

Faremos uma aplicação dos modelos de mudança de regimes em uma série temporal com dinâmica autoregressiva, a série do "spread" Diário entre dois títulos da dívida externa brasileira, o C-Bond e o Par-Bond.

O spread diário é definido como o quociente entre os preços de mercado, apurados no fechamento dos mesmos, dos respectivos títulos. Sejam $c_{t}$ o preço de mercado apurado no fechamento do C-Bond no dia $t$ e $p_{t}$ o preço de mercado apurado no fechamento do Par-Bond no dia $t$. Então, o Spread neste dia é dado por:

$$
y_{t}=\frac{c_{t}}{p_{t}}
$$


A série estudada compreende o período entre 21/01/94 e 13/03/96, perfazendo um total de 476 observações. A Figura 8.1 apresenta o gráfico da série.

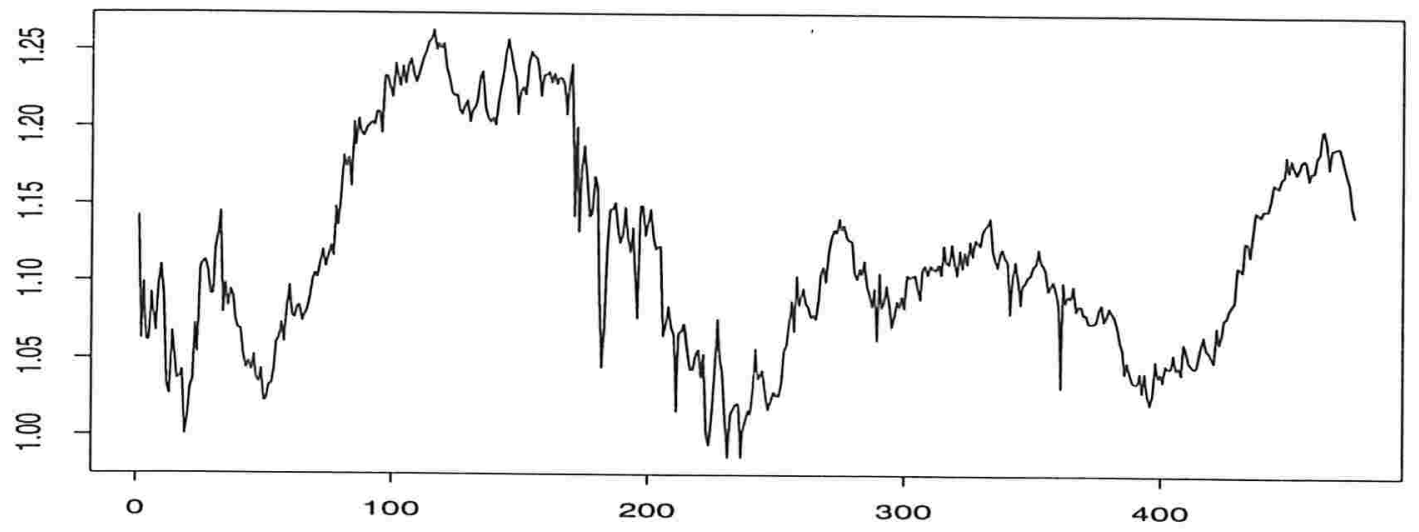

Figura 8.1: Gráfico da Série Spread Diário.

Segundo a análise desta série encontrada em Rabi (1998), "há uma tendência do spread oscilar em torno de um nível médio $(=1.100)$, apresentando porém uma dinâmica autoregressiva na medida em que os afastamentos e os retornos ao nível médio da série ocorrem através de mini-tendências." Esta característica é justificada pela natureza dos dois títulos, que são influenciados conjuntamente por fatores que promovem as alterações.

Rabi (1998) destaca a existência de 3 regimes diferentes:

- o primeiro quando a série oscila num patamar inferior ao nível médio global da série (pequena duração);

- o segundo quando a série oscila ao redor do nível médio global de série (maior duração);

- o terceiro quando as oscilações estão ao redor de um patamar superior ao nível médio global (pequena duração).

Utilizamos dois tipos de abordagem para modelar a série do spread:

a) Aplicar ondaletas às probabilidades associadas a valores da matriz $F_{t}$ (modelos do Capítulo 5);

b) aplicar ondaletas diretamente na matriz do sistema $F_{t}$ (modelos do Capítulo 6). Os resultados encontrados utilizando estes modelos não foram satisfatórios em relação às estimativas das séries. Omitimos a análise realizada. 
a) Ondaletas aplicadas à probabilidades

Supomos a existência de dois estados para a matriz $F_{t}, F_{t}=1$ com probabilidade $P_{1}(t)$ e $F_{t}=0$ com probabilidade $P_{2}(t)$. A probabilidade $P_{1}(t)$ será modelada por uma soma de ondaletas, considerando três coeficientes, $\beta_{1}$, $\beta_{2}$ e $\beta_{3}$ associados. Utilizamos 4 tipos de ondaletas diferentes, Haar, Chapéu Mexicano, Morlet e Shannon. Para cada modelo, realizamos 1000 réplicas bootstrap com o objetivo de obter a média e o erro padrão das estimativas.

A Tabela 8.1 apresenta os valores dos parâmetros para todos os modelos. Observamos que os valores estimados se encontram dentro do intervalo (média erro padrão, média + erro padrão) obtido pelo método bootstrap.

O modelo com maior valor da função de máxima verossimilhança foi o que utilizou a ondaleta Chapéu Mexicano. A Figura 8.2 apresenta os gráficos das séries estimadas, estados estimados e as probabilidades modeladas pelas ondaletas. $\mathrm{Pa}$ ra todos os modelos, os valores estimados para a série foram muito parecidos com a própria série e as estimativas do estado confirmam a hipótese autoregressiva dos dados.

Observamos que para as ondaletas Morlet e Shannon, as probabilidades $P_{1}(t)$ e $P_{2}(t)$ apresentam uma variação ao longo do tempo acompanhando as mudanças na série. A ondaleta Haar estima as duas probabilidades em torno de 0.5 ao longo de todo o tempo e a ondaleta Chapéu Mexicano estima $P_{1}(t)=1 \mathrm{em}$ todo o intervalo de tempo, indicando um único valor constante para $F_{t}$. Para todos os modelos, as probabilidades filtradas são iguais a 1 para $P_{1}(t)$ e 0 para $P_{2}(t)$.

A Figura 8.3 apresenta os histogramas das 1000 amostras bootstrap para cada parâmetro e cada modelo. Observamos em todos os modelos que houve uma concentração dos valores amostrados em torno de um único intervalo para os parâmetros $G_{t}, V_{t}$ e $W_{t}$. Para os coeficientes de ondaletas, principalmente para as ondaletas Morlet e Shannon, houve grande dispersão, com grupos isolados. Precisaríamos de mais estudos do funcionamento das amostras bootstrap para modelos com ondaletas para alguma explicação sobre a distribuição desses parâmetros.

Os modelos utilizados por Rabi (1998) têm o número de parâmetros variando de 3 a 13. O melhor modelo encontrado por ele teve o valor do AIC igual a -2770.0 com 7 parâmetros. O modelo utilizando a ondaleta Chapéu Mexicano teve o valor do AIC igual a -2514.5446 utilizando 6 parâmetros. 
Tabela 8.1: Resultados obtidos para a Série Spread utilizando os modelos Haar, Chapéu Mexicano, Morlet e Shannon, respectivamente (Capítulo 5).

\begin{tabular}{|c|c|c|c|c|}
\hline Parâmetros & Haar & Chapéu & Morlet & Shannon \\
\hline log veros & 933.7208 & 1263.2708 & 944.1408 & 975.1024 \\
\hline $\begin{array}{c}G_{t} \\
\text { média } G_{t} \\
\operatorname{dp} G_{t}\end{array}$ & $\begin{array}{l}0.99997 \\
0.99449 \\
0.01079\end{array}$ & $\begin{array}{l}0.99997 \\
0.99959 \\
0.00229\end{array}$ & $\begin{array}{l}0.99997 \\
0.99953 \\
0.00085\end{array}$ & $\begin{array}{l}0.99997 \\
0.99755 \\
0.00586\end{array}$ \\
\hline $\begin{array}{c}V_{t} \\
\text { média } V_{t} \\
\operatorname{dp} V_{t}\end{array}$ & $\begin{array}{l}0.000073 \\
0.001271 \\
0.001603\end{array}$ & $\begin{array}{l}0.000073 \\
0.001064 \\
0.005051\end{array}$ & $\begin{array}{l}0.000073 \\
0.000724 \\
0.000889\end{array}$ & $\begin{array}{l}0.000073 \\
0.001122 \\
0.012454\end{array}$ \\
\hline $\begin{array}{c}W_{t} \\
\text { média } W_{t} \\
\operatorname{dp} W_{t}\end{array}$ & $\begin{array}{l}0.000168 \\
0.023087 \\
0.050065\end{array}$ & $\begin{array}{l}0.000168 \\
0.001473 \\
0.006130\end{array}$ & $\begin{array}{l}0.000168 \\
0.003529 \\
0.044652\end{array}$ & $\begin{array}{l}0.000168 \\
0.014013 \\
0.043338\end{array}$ \\
\hline $\begin{array}{c}\beta_{1} \\
\text { média } \beta_{1} \\
\operatorname{dp} \beta_{1}\end{array}$ & $\begin{array}{c}0.044288 \\
-0.688403 \\
0.266128\end{array}$ & $\begin{array}{c}36.3778 \\
36.1560 \\
1.342505\end{array}$ & $\begin{array}{c}-0.569695 \\
-0.620711 \\
0.040323\end{array}$ & $\begin{array}{r}-1.358127 \\
-7.518371 \\
8.774905\end{array}$ \\
\hline $\begin{array}{c}\beta_{2} \\
\text { média } \beta_{2} \\
\operatorname{dp} \beta_{2}\end{array}$ & $\begin{array}{c}-0.005694 \\
-1.024488 \\
0.370078\end{array}$ & $\begin{array}{c}-14.1246 \\
-14.07981 \\
0.375781\end{array}$ & $\begin{array}{c}-0.223273 \\
-0.305205 \\
0.064707\end{array}$ & $\begin{array}{r}0.128090 \\
-3.549072 \\
5.092836\end{array}$ \\
\hline $\begin{array}{c}\beta_{3} \\
\text { média } \beta_{3} \\
\operatorname{dp} \beta_{3}\end{array}$ & $\begin{array}{l}0.068327 \\
0.050939 \\
0.006289\end{array}$ & $\begin{array}{c}-9.3370 \\
-9.2019 \\
1.1255\end{array}$ & $\begin{array}{c}-0.499720 \\
-0.535309 \\
0.028080\end{array}$ & $\begin{array}{c}-0.949290 \\
-2.978736 \\
3.238122\end{array}$ \\
\hline
\end{tabular}



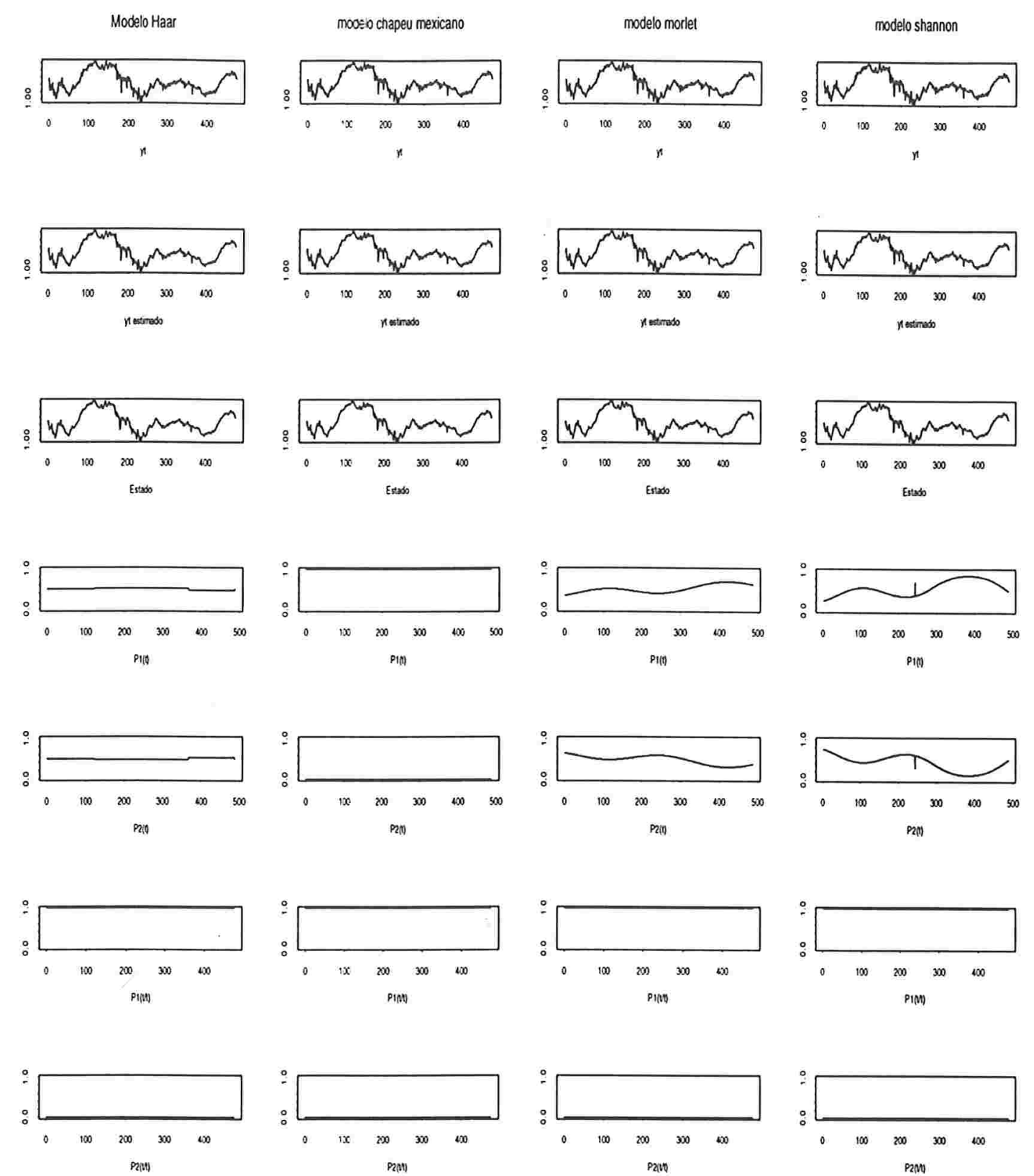

Figura 8.2: Gráficos das estimativas da série, do estado, das probabilidades $P 1(t)$ e $P 2(t)$ e das probabilidades filtradas $P 1(t \mid t)$ e $P 2(t \mid t)$ para os modelos com ondaleta Haar, Chapéu Mexicano, Morlet e Shannon. 

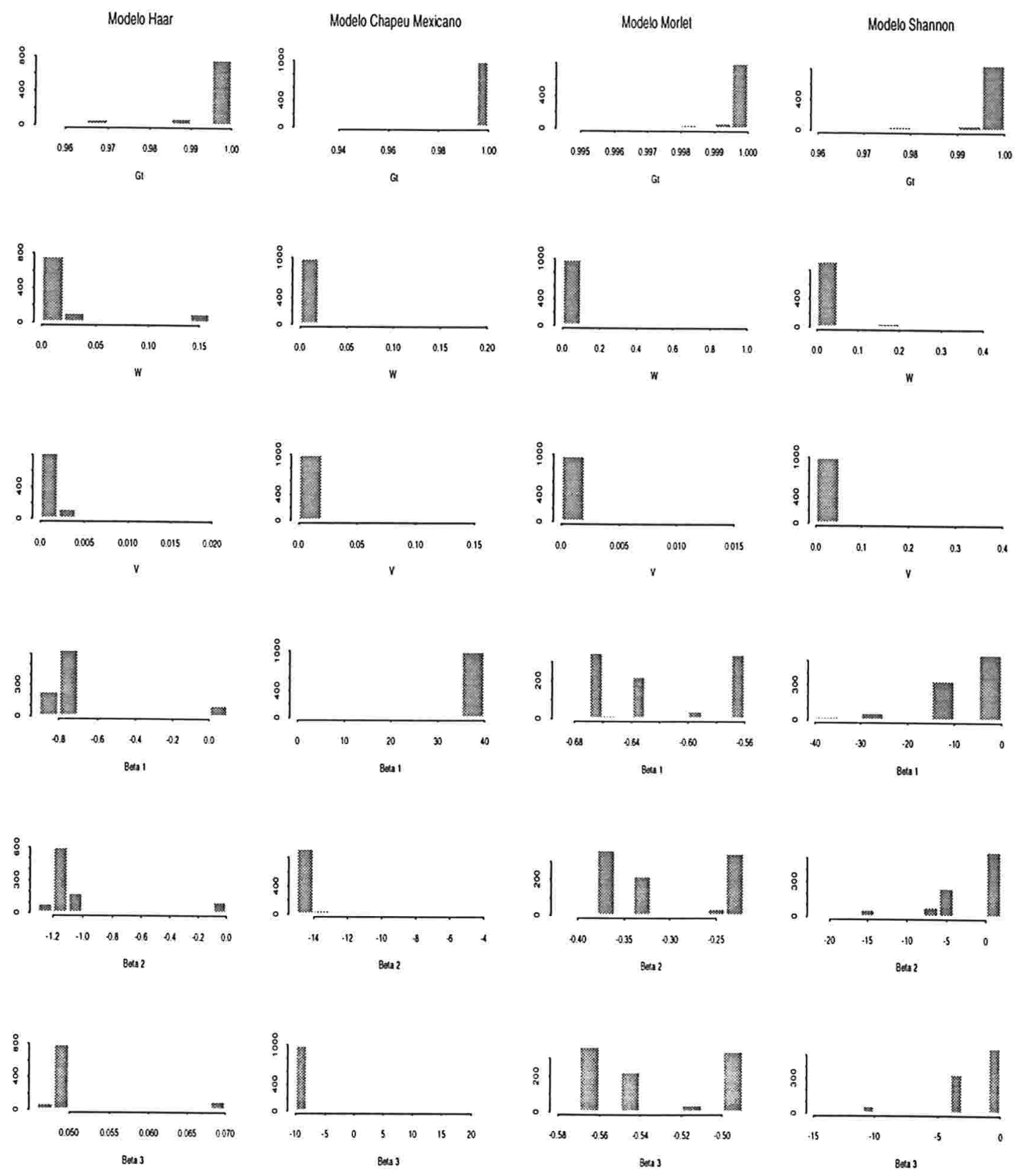

Figura 8.3: Histogramas das amostras bootstrap dos parâmetros $G, W, V, \beta_{1}, \beta_{2}$ e $\beta_{3}$ para os modelos com ondaleta Haar, Chapéu Mexicano, Morlet e Shannon. 


\subsection{Conclusão}

Este trabalho, dentro do rico universo de combinar as técnicas estatísticas Modelos de Espaço de Estados e Ondaletas, foi um início de descobertas e ponto de partida para explorações futuras.

Abordamos a utilização de quatro ondaletas que possuem forma analítica, nada dizendo sobre outras sem forma analítica, como por exemplo, as Daublets, Symmlets e Coiflets, que certamente têm suas especificidades.

O Capítulo 4 apresentou ondaletas na Matriz de Transição. Simulamos alguns exemplos de séries autoregressivas com parâmetro variando no tempo. Iniciamos neste capítulo a utilização da técnica de encolhimento de ondaletas que pode vir a ser melhor estudada posteriormente.

Uma contribuição importante foi a metodologia proposta no Capítulo 5 para modelar probabilidades associadas a possíveis valores da matriz do sistema $F_{t}$, conduzindo a uma solução para séries com mudança de regime.

As idéias desenvolvidas no Capítulo 6 mereceriam um estudo de simulação de possíveis modelos para tais condições.

Não abordamos em nenhum momento previsão para os modelos propostos.

Conseguimos obter formas fechadas para os estimadores, mas encontramos algumas dificuldades para a implementação computacional. Programamos no Fortran 90 a estimação de parâmetros via Algoritmo EM e utilização do Filtro de Kalman (Capítulos 4, 5 e 6). Encontramos dificuldades computacionais de ordem de convergência e de resolução de sistemas não lineares (Capítulo 5). No Capítulo 6 , a desconfiança de estarmos trabalhando com um modelo não observável nos levou a uma tentativa frustrada de prova analítica e à utilização da verossimilhança perfilada como solução.

Muito tempo foi gasto na elaboração dos programas e tentativas de aplicação. Algumas técnicas foram pouco exploradas e a parte prática deve ser um capítulo iniciado a partir de agora.

A utilização do bootstrap como uma ferramenta de obtenção dos erros padrões dos estimadores é uma solução de fácil implementação.

Concluímos este trabalho com a certeza que muita coisa há de ser descoberta com a combinação de técnicas já existentes. Em particular, a ferramenta Ondaleta é muito poderosa e útil para o desenvolvimento da Estatística, principalmente em modelos que apresentam parâmetros com variação temporal. 


\section{Referências Bibliográficas}

Agresti, A. (1990). Categorical Data Analysis. John Wiley e Sons, New York.

Anderson, B. D. O. e Moore, J. B. (1979). Optimal Filtering. Prentice Hall, Englewood Cliffs, N. J. .

Bell, W. e Hillmer, S. (1991). Initializing the Kalman Filter for Nonstationary Time Series Models. Journal of Time Series Analysis, 12(4), 283-300.

Boyles, R. A. (1983). On the Convergence of the EM Algorithm. Journal of the Royal Statistical Society, Series B, 45(1), 47-50.

Carlin, B. P., Polson, N. G. e Stoffer, D. S. (1992). A Monte Carlo Approach to Nonnormal and Nonlinear State-Space Modeling. Journal of the American Statistical Association, 87(418), 493-500.

Carter, C. K. e Kohn, R. (1994). On Gibbs Sampling for State-Space Models. Biometrika, 81(3), 541-553.

Chan, L. K. e Mak, T. K. (1979). Maximum Likelihood Estimation of a Linear Structural Relationship with Replication. Journal of the Royal Statistical Society, Series B, 41(2), 263-268.

Chui, C. K. (1992). An Introduction to Wavelets. Academic Press, San Diego.

Cordeiro, G. (1992). Introdução à Teoria de Verossimilhança. X Simpósio Nacional de Probabilidade e Estatística, Rio de Janeiro, RJ.

Dahlhaus, R. (1993a). Fitting Time Series Models to Nonstationary Processes. Technical Report Beiträge zur Statistik 4, Universität Heidelberg.

Dahlhaus, R. (1993b). Maximum Likelihood Estimation and Model Selection for Locally Stationary Processes. Journal of Nonparametric Statistics, to appear. 
Dahlhaus, R. (1997a). Fitting Time Series Models to Nonstationary Processes. The Annals of Statistics, 25(1), 1-37.

Dahlhaus, R. (1997b). The Analysis of Nonstationary Time Series and Curve Estimation whith Locally Stationary Models. Institute of Statistical Sciences Academia Sinica.

Dahlhaus, R., Neumann, M. H. e von Sachs, R. (1997). Non Linear Wavelet Estimation of the Time-Varying Autoregressive Processes. Bernoulli, tentatively accepted.

Daubechies, I. (1992). Ten Lectures on Wavelets. SIAM , Philadelphia.

Davison, A. C. e Hinkley, D. V. (1997). Bootstrap Methods and their Application. Cambridge University Press, United Kingdom.

de Jong, P. (1988). The Likelihood for a State Space Model. Biometrika, 75(1), 165-169.

de Jong, P. e Chu-Chun-Lin, S. (1994a). Stationary and Nonstationary State Space Models. Journal of Time Series Analysis, 15(2), 151-166.

de Jong, P. e Chu-Chun-Lin, S. (1994b). Fast Likelihood and Prediction for Nonstationary State Space Models. Biometrika, 81(1), 133-142.

Dempster, A. P., Laird, N. M. e Rubin, D. B. (1977). Maximum Likelihood from Incomplete Data via the EM Algorithm. Journal of the Royal Statistical Society, Series B, 39, 1-38.

Donoho, D. L. e Johnstone, I. M. (1995). Adapting to Unkown Smoothness via Wavelet Shrinkage. Journal of the American Statistical Association, 90(432), $1200-1224$.

Donoho, D. L., Johnstone, I. M., Kerkyacharian, G. e Picard, D. (1995). Wavelet Shrinkage: Asymptopia? Journal of the Royal Statistical Society, Series B, 57, 301-337.

Donoho, D. L., Johnstone, I. M., Kerkyacharian, G. e Picard, D. (1996). Density Estimation by Wavelet Thresholding. The Annals of Statistics, 24(2), 508-539. 
Durbin, J. e Koopman, S. J. (1996). Monte Carlo Maximum Likelihood Estimation for Non-Gaussian State Space Models. Technical report, London School of Economics and Political Science, London.

Efron, B. (1979). Bootstrap Methods: Another Look at the Jackknife. The Annals of Statistics, 7, 1-26.

Efron, B. e Tibshirani, R. J. (1993). An Introduction to the Bootstrap. Chapman \& Hall, London.

Fahrmeir, L. (1992). New Directions in Time Series Analysis, Part I, chapter State Space Modelling and Conditional Mode Estimation for Categorical Time Series, pages 87-109. Springer, New York.

Franco, G. (1998). Bootstrap em Modelos Estruturais - Construção de Intervalos de Confiança e Testes de Hipóteses . Ph.D. thesis, DEE-PUC, Rio de Janeiro, RJ.

Gómez, V. e Maravall, A. (1994). Estimation, Prediction and Interpolation for Nonstationary Series with the Kalman Filter. Journal of the American Statistical Association, 89(426), 611-624.

Gupta, N. K. e Mehra, R. K. (1974). Computational Aspects of Maximum Likelihood Estimation and Reduction in Sensitivity Function Calculations. IEEE Transactions on Automatic Control, 19, 774-783.

Hamilton, J. D. (1989). A new Approach to the Economic Analysis of Nonstationary Time Series and the Business Cycle. Econometrica, 57(2), 357-384.

Hamilton, J. D. (1994). Time Series Analysis. Princeton University Press, Princeton.

Harrison, P. J. e Stevens, C. F. (1976). Bayesian Forecasting. Journal of the Royal Statistical Society, Series B, 38, 205-247.

Harvey, A. C. (1984). A Unified View of Statistical Forecasting Procedure. Journal of Forecasting, 3, 245-275.

Harvey, A. C. (1989). The Econometric Analysis of Time Series. Cambridge University Press, Cambridge. 
Harvey, A. C. (1990). Forecasting, Structural Time Series Models and the Kalman Filter. Cambridge University Press, Cambridge.

Harvey, A. C. e Peters, S. (1990). Estimation Procedures for Structural Time Series Models. Journal of Forecasting, 9, 89-108.

Harvey, A. C. e Phillips, G. D. A. (1979). Maximum Likelihood Estimation of Regression Models with Autoregressive-Moving Average Disturbances. Biometrika, 66(1), 49-58.

Hubbard, B. B. (1996). The World According to Wavelets. A. K. Peters, Wellesley.

Jawerth, B. e Sweldens, W. (1994). An Overview of Wavelet Based Multiresolution Analysis. SIAM Review, 36, 377-412.

Kim, C. J. (1994). Dynamic Linear Models with Markov-Switching. Journal of Econometrics, 60, 1-22.

Kitagawa, G. (1981). A Nonstationary Time Series Model and its Fitting by a Recursive Filter. Journal of Time Series Analysis, 2(2), 103-116.

Kitagawa, G. e Gersch, W. (1996). Smoothness Priors Analysis of Time Series. Lecture Notes in Statistics, 116. Springer Verlag, New York.

Ljung, L. e Caines, P. E. (1979). Asymptotic Normality of Prediction Error Estimators for Approximate System Models. Stochastics, 3, 29-46.

Louis, T. A. (1982). Finding the Observed Information Matrix when Using the EM Algorithm. Journal of the Royal Statistical Society, Series B, 44(2), 226233.

Meilijson, I. (1989). A Fast Improvement to the EM Algoritm on its Own Terms. Journal of the Royal Statistical Society, Series B, 51(1), 127-138.

Meinhold, R. J. e Singpurwalla, N. D. (1989). Robustification of Kalman Filter Models. Journal of the American Statistical Association, 84(406), 479-486.

Meyer, Y. (1993). Wavelets: Algoritms and Applications. SIAM, Philadelphia.

Morettin, P. A. (1997a). Ondaletas e Seus Usos na Estatística. VII Escola de Séries Temporais e Econometria, Canela, RS. 
Morettin, P. A. (1997b). Wavelets in Statistics. Resenhas IME-USP, 3(2), 211272.

Murteira, B. J. F., Müller, D. A. e Turkman, K. F. (1993). Análise de Sucessões Cronológicas. Mc Graw-Hill, Portugal.

Ogden, R. T. (1997). Essential Wavelets for Statistical Applications and Data Analysis. Birkhäuser, Boston, USA.

Press, W. H., Teukolsky, S. A., Vetterling, W. T. e Flannery, B. P. (1992). Numerical Recipes in Fortran 77, The Art of Scientific Computing, Vol. 1. Cambridge University Press, Cambridge.

Rabi, L. A. J. (1998). Modelos de Mudanças Markovianas de Regimes Aplicados a Séries Temporais Financeiras. Dissertação de Mestrado, IME-USP, São Paulo.

Richards, F. S. G. (1961). A Method of Maximum Likelihood Estimation. Journal of the Royal Statistical Society, Series B, 23, 469-475.

Shumway, R. H. e Stoffer, D. S. (1982). An Approach to Time Series Smoothing and Forecasting Using the EM Algotithm. Journal of Time Series Analysis, 3(4), 253-264.

Shumway, R. H. e Stoffer, D. S. (1991). Dynamic Linear Models with Switching. Journal of the American Statistical Association, 86(415), 763-769.

Shumway, R. H., Olsen, D. E. e Levy, L. J. (1981). Estimation and Tests of Hypotheses for the Inicial Mean and Covariance in the Kalman Filter Model. Communications Statistics-Theory Methods, 10(16), 1625-1641.

Souza, R. C. (1989). Modelos Estruturais para Previsão de Séries Temporais: Abordagem Clássica e Bayesiana. XVII Colóquio Brasileiro de Matemática, IMPA, Rio de Janeiro, RJ.

Spall, J. C. e Wall, K. D. (1984). Asymptotic Distribution Theory for the Kalman Filter State Estimator. Communications Statistics-Theory Methods, 13(16), 1981-2003.

Stoffer, D. S. e Wall, K. D. (1991). Bootstrapping State-Space Models: Gaussian Maximum Likelihood Estimation and the Kalman Filter. Journal of the American Statistical Association, 86(416), 1024-1033. 
Strang, G. (1993). Wavelet Transforms Versus Fourier Transforms. Bulletin of the American Mathematical Society, 28(2), 288-305.

Tanner, M. A. (1996). Tools for Statistical Inference Methods for the Exploration of Posterior Distributions and Likelihood Functions. Springer-Verlag, New York.

Vidakovic, B. e Müller, P. (1994). Wavelets for Kids: A Tutorial Introduction. Technical Report 94-A-13, ISDS, Duke University, Durham, North Carolina.

West, M. e Harrison, J. (1997). Bayesian Forecasting and Dynamic Models. Springer-Verlag, New York.

Wu, C. F. J. (1983). On the Convergence Properties of the EM Algorithm. The Annals of Statistics, 11(1), 95-103. 